\title{
WestVirginiaUniversity
}

THE RESEARCH REPOSITORY @ WVU

Graduate Theses, Dissertations, and Problem Reports

2005

\section{Computer Aided Home Energy Management system}

Asawari A. Moholkar

West Virginia University

Follow this and additional works at: https://researchrepository.wvu.edu/etd

\section{Recommended Citation}

Moholkar, Asawari A., "Computer Aided Home Energy Management system" (2005). Graduate Theses, Dissertations, and Problem Reports. 1638.

https://researchrepository.wvu.edu/etd/1638

This Thesis is protected by copyright and/or related rights. It has been brought to you by the The Research Repository @ WVU with permission from the rights-holder(s). You are free to use this Thesis in any way that is permitted by the copyright and related rights legislation that applies to your use. For other uses you must obtain permission from the rights-holder(s) directly, unless additional rights are indicated by a Creative Commons license in the record and/ or on the work itself. This Thesis has been accepted for inclusion in WVU Graduate Theses, Dissertations, and Problem Reports collection by an authorized administrator of The Research Repository @ WVU. For more information, please contact researchrepository@mail.wvu.edu. 


\title{
Computer Aided Home Energy Management System
}

\author{
by \\ Asawari A Moholkar \\ Thesis submitted to the College of Engineering and Mining Resources \\ at West Virginia University \\ in partial fulfillment of the requirements \\ for the degree of \\ Master of Science \\ in \\ Electrical Engineering \\ Approved by \\ Dr. Powsiri Klinkhachorn, Committee Chairperson \\ Dr. Ali Feliachi \\ Dr. Xin Li \\ Dr. Hyungna Oh
}

Lane Department of Computer Science and Electrical Engineering

\section{Morgantown, West Virginia \\ 2005}

Keywords:

Energy Management, Peak Load Management, Real Time Pricing, Price

Responsive Load, Fuzzy Logic, Home Automation

Copyright 2005 Asawari A. Moholkar 


\title{
ABSTRACT \\ Computer Aided Home Energy Management System
}

\author{
Asawari A. Moholkar
}

As the electricity demand approaches generation capacity, the operating reserve capacity of the power system shrinks, causing an increased risk of outages. Demand Side Management (DSM) programs are designed to modify the demand profile to mitigate the peak demand. In newly deregulated electricity markets, price spikes are often observed at peak demand periods. Thus, price is a good indicator of the demand condition of a power system. Because of the hockey-stick shape of the aggregate supply curve, even a small reduction in the peak demand can result in a large change in price, even eliminating price spikes.

The Price-Responsive Load Management (PRLM) program forms a foundation for the development of the Computer-Aided Home Energy Management (CAHEM) system. As customer acceptance is a very important factor in the successful implementation of the CAHEM system, it is necessary to include customer preferences in the program design. The load shifting algorithm tries to optimize both customer satisfaction and peak load reduction based on a rule-based fuzzy controller. The CAHEM system enables the residential customer to smartly control their demand profile as a reaction to changes in the spot price of electricity. The residential electricity demand constitutes energy consumptions due to air conditioners, water heaters, dishwashers, washers/dryers, etc.

The aggregated impacts of PRLM with the CAHEM system are evaluated using temperature, load, and price data for the summer of 1999 in the Pennsylvania-New Jersey-Maryland (PJM) market. The simulation results indicate a three percent reduction in aggregated peak load is achievable with the CAHEM system assumed to be installed at only forty percent of the total houses. 


\section{ACKNOWLEDGMENTS}

First and foremost, I would like to thank God for giving me strength and courage and for keeping me surrounded by dedicated teachers, loving family and friends throughout my entire life.

I have been privileged to have Dr. Powsiri Klinkhachorn as my advisor. I would like to express my deep gratitude and respect to him for his excellent guidance, continuous encouragement, and support through the last two years. His attention to detail, love for perfection, and dedication towards work has always motivated me to persistently give my best. When faced with difficulties or dilemmas, both in research as well as in my personal life, I always believed I could rely on him for guidance. I am really indebted to him for making my master's experience a memorable one.

The research presented in this thesis was sponsored in part by US DoE EPSCoR WV State Implementation Award. I am grateful to Dr. Ali Feliachi for giving me the opportunity to work with the Advanced Power and Electricity Research Center (APERC) for the last two years. I express my sincere thanks to Dr. Hyungna Oh for her expert guidance and for devoting her time for long, fruitful discussions. I thank Dr. Kenneth Means for helping me in developing thermodynamic models. I wish to acknowledge and thank the APERC team for their support, encouragement, interactive discussions, as well as criticism, which helped me improve my research work and personal skills. I sincerely thank Elizabeth Compton for editing this thesis.

I would like to take this opportunity to sincerely thank Dr. Xin Li and Dr. Donald Adjeroh for their support and encouragement during my initial struggling period at WVU. I am grateful to them for giving me a chance to study under their guidance. I would like to specially thank all the professors, staff, and the systems group for their excellent work and support.

I have been fortunate to have caring friends around me at all the times. I thank my friends for their continuous support, both from India as well as from the United States. I wish them success in their future endeavors.

I am forever grateful to my family for their incredible love, blessings, inspiration, support, efforts, and sacrifices, without which I would not have come this far. Finally, to my precious fiancé, Kaustubh, you have always been very caring, supportive, and encouraging. Thanks for understanding me and sharing joys as well as worries. To all of you, I dedicate my thesis. 


\section{TABLE OF CONTENTS}

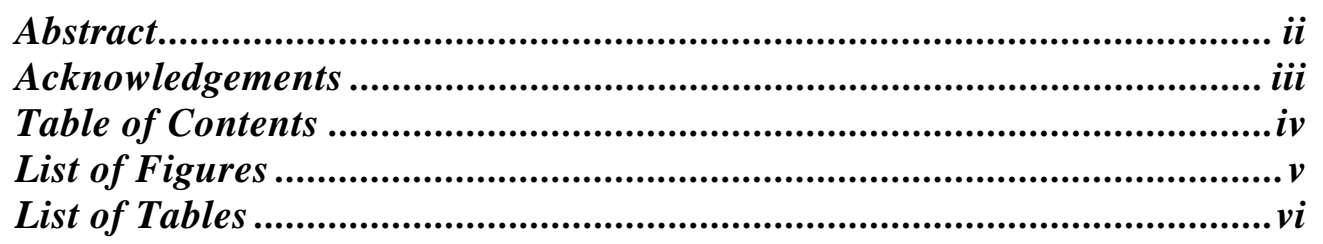

Chapter 1 - Introduction................................................................1

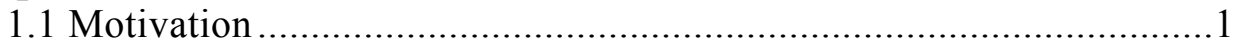

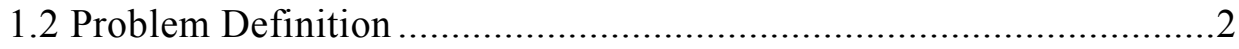

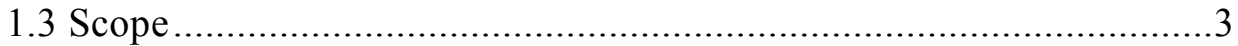

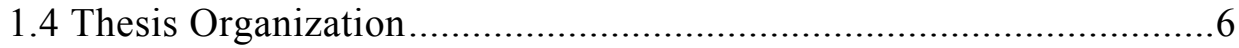

Chapter 2 - Background and Related Work.........................................

2.1 Fundamentals of Power System Economics ................................... 7

2.2 Peak Load Management ..........................................................11

2.3 Residential Peak Load Management Programs ...............................13

2.3.1 Direct Load Control........................................................13

2.3.2 Price Responsive Load Management ................................. 16

2.3.2.1 Time Of Use ................................................... 17

2.3.2.2 Real Time Pricing ................................................ 19

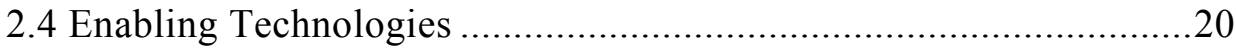

2.4.1 State of The Art in Home Networking Technology .............23

2.4.1.1 CEBus .................................................................23

2.4.1.2 LonWorks ..........................................................24

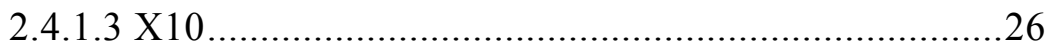

2.4.2 Load Controllers........................................................28

Chapter 3 - Computer-Aided Home Energy Management System ........30

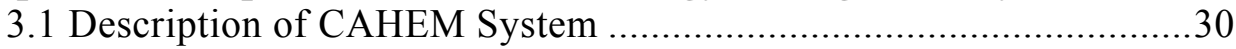

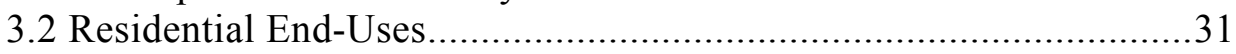

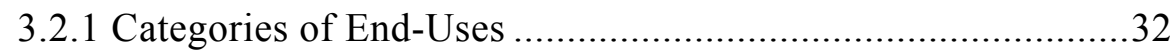

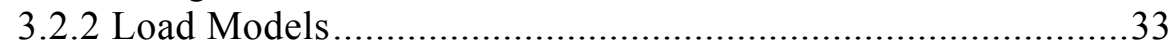

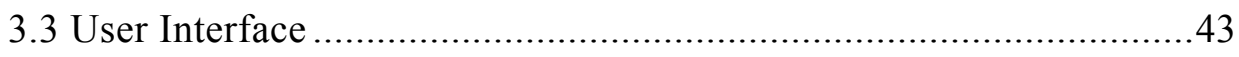

3.3.1 Hardware Components .................................................4 43

3.3.2 User Interface Software ....................................................43

3.4 Price Responsive Load Management Strategy ...............................50

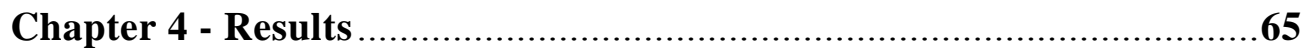

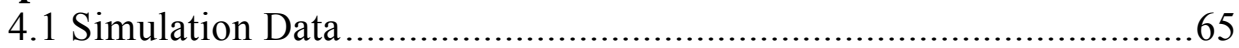

4.2 Results at Individual House-level .............................................68

4.3 Results at Aggregated-level................................................. 70

Chapter 5 - Conclusion and Future Work .....................................76

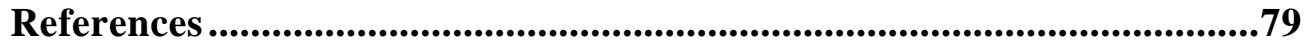




\section{LIST OF FIGURES}

Figure 1: An example of load-duration curve ……................................................ 8

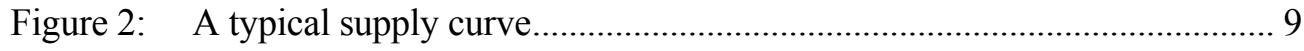

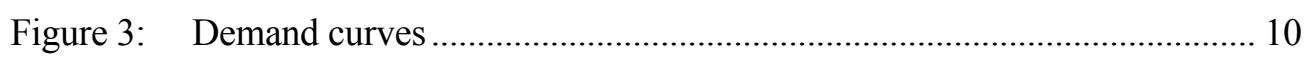

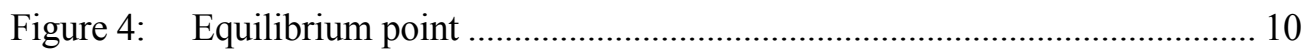

Figure 5: Projected effect of DLC on load-duration curve ..................................... 14

Figure 6: Conventional Direct Load Control............................................................ 15

Figure 7: Projected effect of PRLM on load-duration curve .................................. 16

Figure 8: Price Responsive Load Management - Increased Customer Choice ...... 17

Figure 9: Alabama Power Company's Residential TOU Schedule - FDT............... 18

Figure 10: Spot Prices (PJM Market on June 26, 1999) ………................................ 20

Figure 11: Overview of communication technologies enabling PRLM..................... 21

Figure 12: Timing relationship of X10 signals............................................................ 26

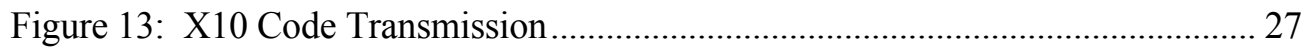

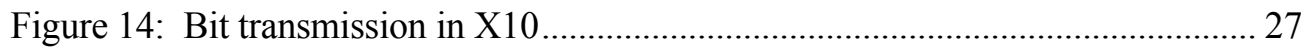

Figure 15: Block Diagram for the CAHEM …............................................................. 30

Figure 16: Breakdown of residential electricity consumption ................................. 32

Figure 17: Categories of residential end-use devices .............................................. 32

Figure 18: Thermal network model for air conditioned house ………………............ 35

Figure 19: Thermal comfort zone _........................................................................... 39

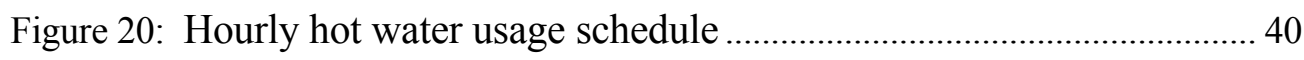

Figure 21: Hourly lighting usage profile................................................................. 42

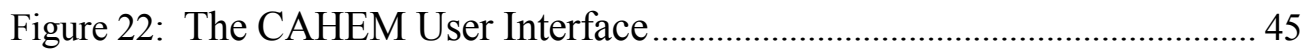

Figure 23: The CAHEM User Interface - Configuration ..................................... 46

Figure 24: The CAHEM User Interface - Main Page ........................................... 47

Figure 25: Appliance-wise electricity consumption profiles............................. 49

Figure 26: Uncertainty in supply and price relation............................................. 50

Figure 27: Membership functions for Hour Classification fuzzy unit .............. 52

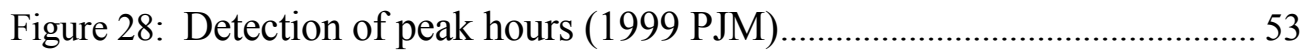

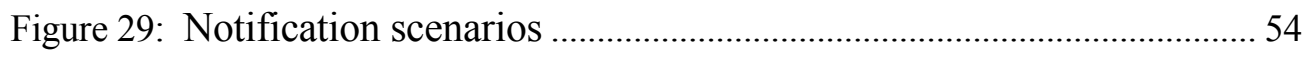

Figure 30: Flowchart for load control with hour-ahead notification ................... 55

Figure 31: Load profile with control based on hour-ahead notification ............ 55 
Figure 32: Flowchart for the appliance control under CAHEM .......................... 56

Figure 33: Load profile with control based on N-hour-ahead notification ....... 57

Figure 34: Membership functions for fuzzy controller during peak hours - a) load,

b) price , c) thermostat setting, d) water heater performance, and

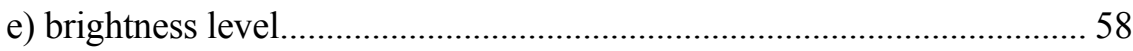

Figure 35: Surface plots for fuzzy controller during peak hours ............................... 61

Figure 36: Membership functions for fuzzy controller during pre-peak hours

a) load, b) price ,and c) thermostat setting .............................................. 63

Figure 37: Surface plot for pre-peak fuzzy controller.......................................64

Figure 38: Load duration curve for the PJM 1999 summer market.................... 65

Figure 39: Price duration curve for the PJM 1999 summer market ..................... 66

Figure 40: Supply curve for the PJM 1999 summer market ................................ 66

Figure 41: Hour classification - a) Super-save mode, b) Economy mode, and c) Comfort mode 67

Figure 42: Results for single housing unit operating in economy mode with hour-ahead notification 68

Figure 43: Results for single housing unit operating in economy mode with

N-hour-ahead notification 69

Figure 44: Distribution of floor area of house classes 70

Figure 45: Load duration curves with hour-ahead notification -

a) complete view, and b) an enlarged view .72

Figure 46: Load duration curves with N-hour-ahead notification -

a) complete view, and b) an enlarged view 73

Figure 47: Effect of CAHEM participation on peak load reduction 75 


\section{LIST OF TABLES}

Table 1: Summary of Residential Direct Load Control programs ................... 15

Table 2: Summary of modes of the CAHEM ..................................................4 44

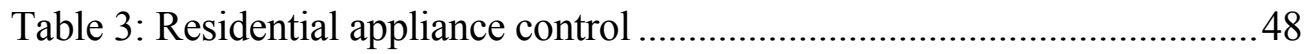

Table 4: Comparison of peak load reduction with various modes ................... 74 


\section{Chapter 1}

\section{Introduction}

\subsection{Motivation}

The need for electricity has been growing rapidly in recent years as a result of economic activities, weather, and population growth. Though technological advances allow the storage of electricity, it is still not viable to store electricity in large quantities. Therefore, electricity must be generated at the moment that it is demanded [1]. Additionally, the electricity demand varies significantly over time on an hourly, daily, as well as seasonal basis [3]. Power systems ${ }^{1}$ often encounter unexpectedly high demand levels due to various reasons, such as extreme weather conditions. The power system operator needs to continuously balance the supply and the demand for reliable power delivery across the transmission grid. Power systems maintain an operating reserve capacity ${ }^{2}$ to supply unexpected excessive demand. As the demand approaches generation capacity, the operating reserve capacity is decreased, which makes the power system susceptible to outages.

A wide range of Demand Side Management (DSM) programs have been proposed by utilities to modify electricity demand profiles. The management of the peak load is one of the most important objectives of DSM programs. The majority of DSM programs are designed for industrial and commercial sectors, due to their significant contribution in peak demand. Though the residential sector contributes nearly $20-25 \%$ of the peak load, it has not yet received enough attention in peak load management. The utilities can widen

\footnotetext{
${ }^{1}$ Power systems include all electrically connected power grids, plus all generators, loads, and control centers associated with them [2].

${ }^{2}$ Operating reserve capacity is the generation capacity in excess of demand which is scheduled to be available on short notice to ensure the reliable power delivery.
} 
the scope of peak load management by exploring the hidden potential of the residential sector.

Direct Load Control (DLC) programs form a class of DSM programs in which the utility remotely controls by either cycling or shutting off certain loads. Demand shaping is achieved by rescheduling various processes or services. In residential DLC programs, the utilities control residential water heaters and air conditioners so as to reduce their usage during peak periods. In DLC programs, load reduction is typically mandatory and is achieved at the cost of the customer's discomfort. Customers are typically rewarded in advance for their participation through discounts or credits on their monthly bill. The customer's acceptance of any such program is determined by balancing the impacts on comfort against the economic incentives. Therefore, to improve customer acceptance of peak load management programs, the utilities need to be more proactive and involve customers in load shifting decisions.

In a traditional electricity market, the retail customers usually face a regulated flat rate for

the electricity consumed. Such regulated prices make the retail customers unaware of the power system condition, and thus, offer very little or no incentive for them to make any efforts to reduce the peak load [2]. If the condition of the power system is communicated to the residential customers through dynamic prices, they can react by shifting or reducing their electricity consumption. A class of such DSM programs based upon the dynamic prices is known as the Price-Responsive Load Management (PRLM) program. The implementation of PRLM has become feasible because of the technological advances in communication, appliance controllers, and advanced metering.

\subsection{Problem Definition}

The spot prices of electricity impart information about the power system condition. High prices and high price volatility occur together in electricity markets. Even a small reduction in peak demand can result in a large reduction in price. This is due to the fact 
that the supply curve ${ }^{3}$ is hockey stick shape [5]. A well-designed PRLM program will allow the residential electricity consumers to reduce or shift their peak consumption automatically in real time with an increase in price.

In the traditional load shedding approach, the consumer reduces electricity usage during peak load hours without any compensating increase in usage during off-peak hours. Thus, load shedding reduces the overall electricity consumption as compared to the non-priceresponsive status quo. It also reduces the total benefits to the consumer as a result of reduced electricity usage from what is required to maintain desired comfort. The loss of total benefits due to load shedding can be minimized if the energy usage is compensated during off-peak hours.

A prime objective of this research is to investigate the potential of the residential PRLM program based on the spot prices. It underlines the following tasks:

1. Development of a price-responsive load shifting algorithm

2. Development of a load model for the residential appliances

3. Design of a user interface to allow customer control

4. Aggregate-level simulations to evaluate peak load reduction

According to Oh [5], only 3 percent reduction in peak load is sufficient to eliminate price spikes. The aggregate-level simulations will investigate if the residential PRLM program is capable of providing the required level of peak load reduction.

\subsection{Scope}

In newly restructured electricity markets, the price spikes have occurred as a result of unexpectedly high demand levels, short-term capacity shortages, as well as lack of price responsive loads. A price-responsive load can provide a natural mechanism for relieving short-term constraints associated with peak load [1]. As the overall electricity demand is

\footnotetext{
${ }^{3}$ Explained in Section 2.1
} 
contributed by industrial, commercial, and residential customers, each of these demand sectors has the potential for making overall demand price-responsiveness. There are a number of available pricing strategies, such as time-of-use and real time prices, which provide dynamic pricing incentives to customers for altering their demands in response to electricity prices.

It is anticipated that price-responsive load management will:

- Improve system reliability

- Mitigate price volatility and price spikes

- Reduce average electricity prices to all customers

In price-responsive load management literature, the residential sector has received relatively little attention. This thesis investigates the potential of residential PRLM programs based on real time spot prices.

The residential PRLM system is composed of the following components:

- Load shifting strategies

- Communication system, including home networking

- User interface for controls

- Appliance controller hardware

- Advanced interval meter

- Mechanism for incentive or credit for load reduction

Development of each component of the residential PRLM system itself is a complex problem. Researchers from various backgrounds, such as communication technologies, power system economics, home networking, control systems, etc.; have been contributing to the development of PRLM systems. The practical implementation of the residential PRLM program requires a complex analysis of the above components, supported by the study of consumer psychology. Consumer awareness and education is a lengthy process which must take place through pilot programs. 
This research is based on the following assumptions:

1. Accurate load and price forecasting algorithms are available.

2. The communication infrastructure which establishes communication between the demand controller and the utility exists.

The Computer Aided Home Energy Management (CAHEM) system developed in this thesis is founded on the concept of PRLM. It enables the residential customers to modify their electricity consumption based on real time spot price signals. It focuses on the following components related to residential PRLM programs:

- Load shifting algorithm -

A rule-based fuzzy controller will determine set points for residential appliances, such as air conditioner, water heater, washer/dryer, dishwasher, and lights, based on spot prices and load predictions, as well as customer preferences.

\section{- CAHEM User Interface -}

The customer will determine preferences for operating residential appliances using the user interface. The CAHEM user interface will allow different modes to reflect the demand elasticity that the customer can offer. The prototype CAHEM system will demonstrate how residential appliances can be controlled in response to price signals. The concept of PRLM will be demonstrated with the X10 home automation technology supporting the appliance controllers, for X10 technology is cost effective and easily accessible.

- Aggregate-level Simulations -

The aggregate level implementation of the CAHEM concept will be simulated for testing purposes. The aggregated electricity consumption from residential units within territory under consideration will be estimated with the help of physically based appliance models. The diversities in operating conditions of the residential appliances and other household parameters affecting electricity demand in different houses will be represented by varying the parameters within acceptable limits. The simulation scenarios will consider different 
customer preferences and the overall participation percentage of the program. The aggregate-level simulations are carried out for the Pennsylvania-New Jersey-Maryland (PJM) market for the summer of 1999.

\subsection{Thesis Organization}

Following Chapter 1, the problem definition and scope, Chapter 2 provides background information and related work to the problem of residential energy management. Chapter 2 focuses on the basics of power system economics and the concept and methods of peak load management. The concepts of price-responsive load management (PRLM) with time of use (TOU) and real time prices (RTP) are introduced. As technology support plays a key role in the implementation of PRLM, various home networking protocols and appliance controllers are explained.

Chapter 3 deals with the Computer Aided Home Energy Management System (CAHEM). It explains the overall concept and components of the CAHEM system. Each component of the system is explained in detail. The categories of residential appliances and their respective load models for calculating hourly electricity consumption are described in this subsection. The second part of this section gives algorithmic details of the load control strategies used in this research. There are two different strategies contingent upon the price signal update. The description of the load control algorithm consists of an explanation of the fuzzy controller parameters, membership functions, and fuzzy rule sets.

Chapter 4 represents the results on a single household level, as well as an aggregate system level, using the Pennsylvania- New Jersey- Maryland (PJM) market data for the summer of 1999. Lastly, Chapter 5 presents the conclusions and future work. 


\section{Chapter 2}

\section{Background and Related Work}

\subsection{Fundamentals of Power System Economics}

Supply and demand are the most fundamental concepts in any market economy. In general, supply refers to the quantity of a product or service that the suppliers are willing to offer at a given price, while demand or load refers to the quantity of a product or service that consumers are not only willing to buy but also have the capacity to buy at the given price [6]. In this thesis, the terms demand and load are used synonymously.

The power system operator must maintain a balance between the supply and the demand. Any imbalance between supply and demand causes the system frequency to deviate from the standard frequency of supply. Several applications require very precise supply frequency for their reliable operation. Along with frequency, the system operator must continue to supply power with the specified voltage. Thus, frequency and voltage are important factors in the definition of demand.

Stoft [2] defines demand specific to the electricity market as,

"The amount of power that would be consumed if system frequency and voltage were equal to their target values for all consumers."

The electricity supply must always be equal to consumption, including power losses during transmission. The real-time demand from demand sectors changes continuously. Traditionally, the characteristics of demand have been described by a load-duration curve, a graph representing the number of hours in a year with the total demand at a particular level [2]. Duration is generally represented in normalized form. A typical load duration curve is shown in Figure 1. 


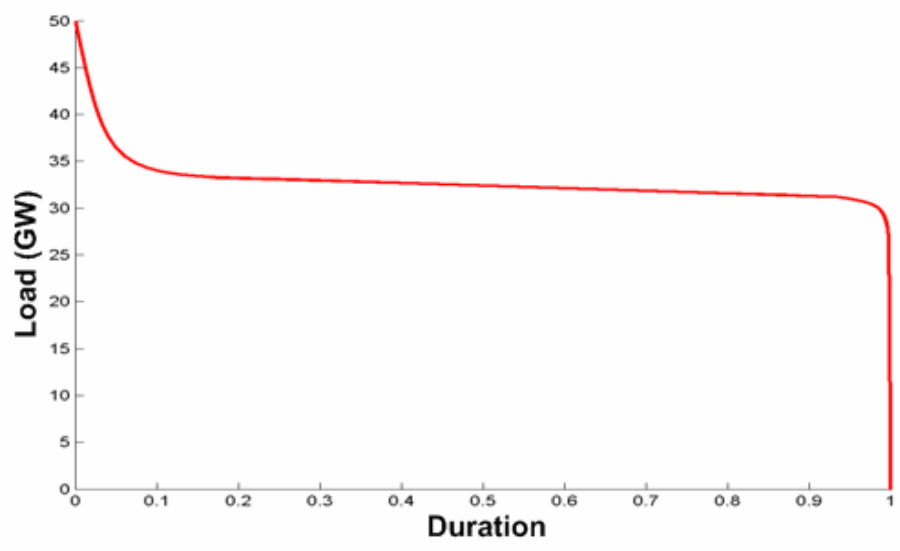

Figure 1: An example of load-duration curve

Peak load is the load during the period when the system demand is relatively high. It is clear from the load-duration curve that peak load appears only for a very short time, and the power system must satisfy this load in real-time. Weather is likely to be the most important drive of peak load. The system peak load is influenced by various other factors including demographics, regional economic activities, and usage behavior, as well as government policies [7]. The load-duration curve in Figure 1 indicates that the power system faces critical hourly demands for a very small duration. For about 90 percent of the duration, the hourly demand is nearly constant.

Power systems are designed to maintain operating reserve capacities to deal with issues related to non-storable nature and requirement of real-time power delivery. Thus, the utilities store generation capacities rather than storing actual energy [3]. The highly efficient and cost-effective power plants operate as the base-load ${ }^{4}$ plants to supply the load at the lowest generation cost. On the other hand, less efficient power plants are reserved as peaking plants, which are operated only to supply additional demand during peak hours at relatively high prices.

\footnotetext{
${ }^{4}$ Base-load is a portion of the system load which is constant at any time. It is essentially the minimum load for the given power system [2].
} 
The load-duration curve completely describes load profile terms of time intervals for each level of load. However, it cannot provide information about the sequence of occurrences for these load levels [2].

Supply and demand are traditionally represented as a function of price and are plotted as supply curves and demand curves. Figure 2 shows a supply curve for an electricity market.

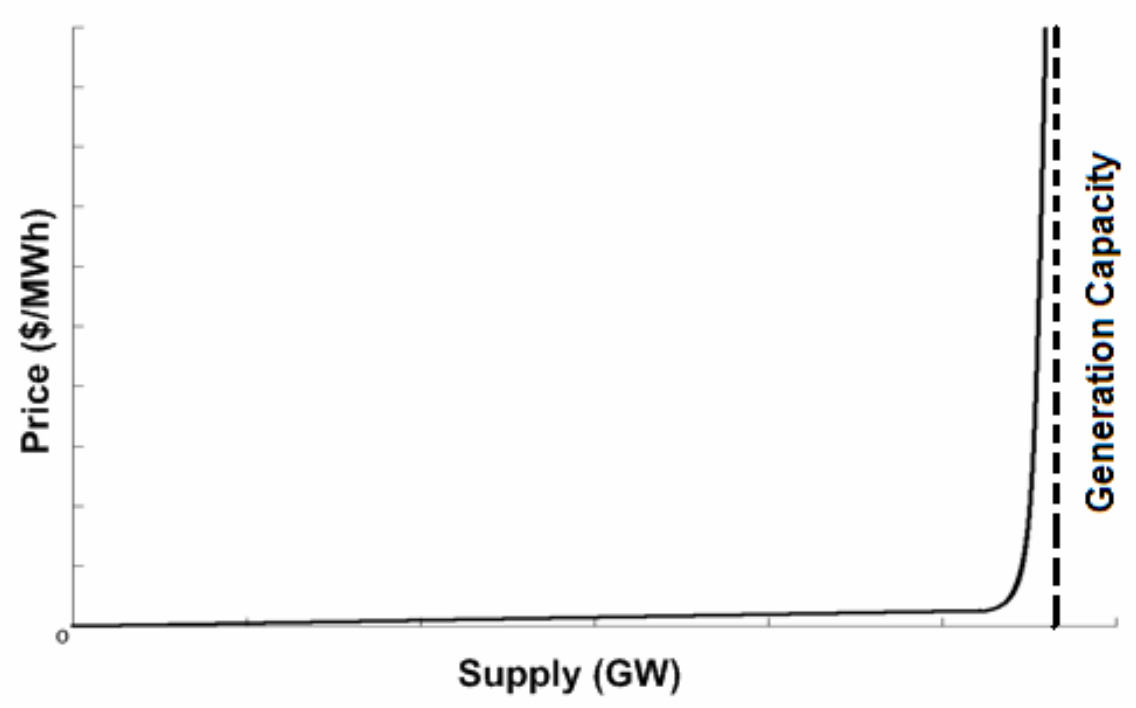

Figure 2: A typical Supply Curve

As shown in the above figure, price is low for a major portion of the supply curve because of the operation of highly efficient base-load plants. The vertical section of the supply curve indicates that even a very small change in supply will cause a very large change in the price of the operating cost for the peaking plants.

In current regulated electricity markets, the residential load usually faces a price that fluctuates very little, for the wholesale price fluctuations are not passed on to retail customers. The residential demand in the current electricity market is not sensitive to hourly price changes, resulting from a lack of market information. Economists define sensitivity of demand to price by price elasticity of demand, or simply, demand elasticity. 
The demand elasticity is given by $\eta=-\frac{\partial D / D}{\partial P / P}$ where, $\Delta D$ is the change in demand caused by a small change in price, $\Delta P[2]$. Demand elasticity plays an important role in peak load management.

Inelastic demand remains nearly constant, even at the highest price. The elastic demand curve tends to have a negative slope as the demand reduces with price increases. The demand curves with inelastic and elastic demand are shown in Figure 3.

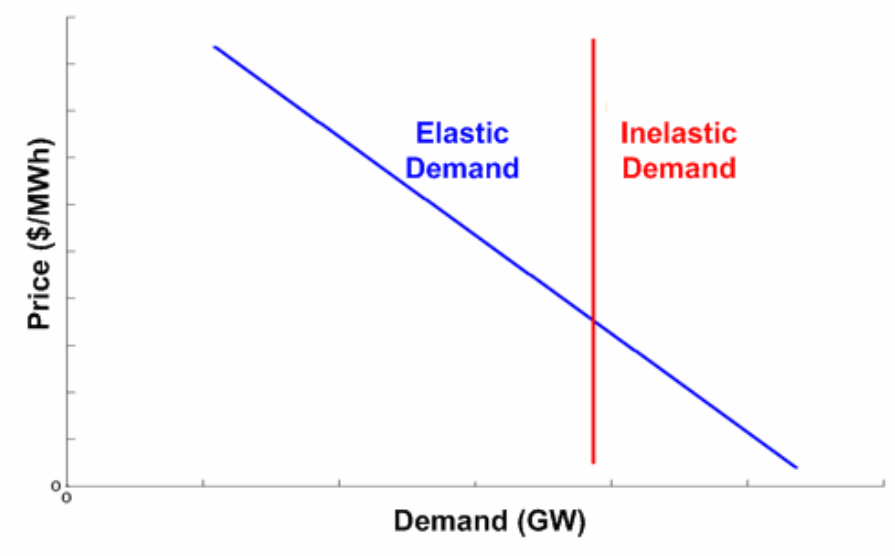

Figure 3: Demand curves

The point where the supply and demand curves intersect is known as the equilibrium point. At the equilibrium, demand equals supply. Figure 4 shows equilibrium for the electricity market.

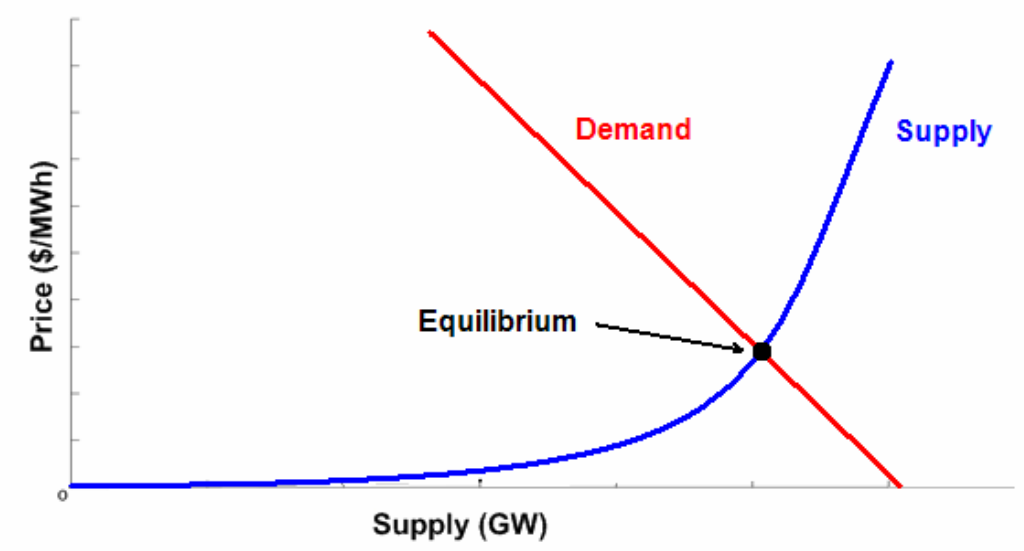

Figure 4: Equilibrium point 
The demand can be made elastic if the price fluctuations are passed to the customer. The dynamic prices, e.g. TOU, Critical Peak Prices (CPP), and RTP, explained in later sections, tend to increase prices when the demand increases. The following section describes the concepts and approaches for peak load management.

\subsection{Peak Load Management}

System reliability, overall efficiency, and operating cost are very critical concerns associated with the use of peaking plants. The operating hours of peaking plants can be reduced if the peak loads are reduced to base-load. The methods of achieving peak load reduction are collectively known as 'Load Management' or 'Peak Load Management'5.

Osborn defines Load Management as

"An economic reduction of electric energy demand during a utility's peak generation periods" [3].

The concept behind load management differs from that of energy conservation. Load Management specifically deals with the strategies to reduce or shift demand from peak to off-peak times, whereas energy conservation aims at reducing aggregate electricity consumption.

\section{Motives behind Peak Load Management}

There are numerous factors that drive the utilities to develop peak load management programs. These factors include system reliability, risk management, system efficiency improvements, and cost reduction [8].

\footnotetext{
5 The terms, Peak Load Management, Load Management, and Demand Side Management are used synonymously in this thesis.
} 


\section{System Reliability}

System reliability is the most important motivation behind the development of peak load management programs. The North American Electric Reliability Council (NERC) defines power system reliability as

"The degree to which the performances of the elements of the electrical system result in power being delivered to consumers within acceptable standards and in the amount desired" [1,7].

The duration and frequency of system interruptions determine system reliability. Power systems are designed with redundancy features and standby or reserve capacity required to meet the demand during emergencies. As the power system approaches a peak load, the reserve capacity may fall below the minimum generation capacity necessary to maintain system reliability. Thus, the likelihood of system outages is extremely high during the peak load times.

\section{System Efficiency}

Efficient utilization of the generation capacity is another important driver for peak load management. The utilities call for the operating reserve when the demand nears the generation capacity. As mentioned previously, the utilities obtain power from small peaker power plants that can start at a moment's notice. Such power plants are far less efficient at power generation. With peaker power plants operating, the overall system efficiency is reduced. The overall system efficiency can be improved if the incidences of operations of peaker power plants are reduced. 


\section{Risk Management}

The risk of financial losses on the part of the load serving entity (LSE) ${ }^{6}$ is threatening when the wholesale price exceeds the price which can be recovered under its contact with a customer. Peak load management can lead to the reduced cost of purchased power as it is being utilized smartly when the price is low.

\section{Cost Avoidance and Reduction}

In general, peak capacity is only utilized for approximately one to two percent of total operating hours in a year. The number of price spikes can be reduced as a result of the reduced operation of expensive peaking plants.

\subsection{Residential Peak Load Management Programs}

Though residential load management is not a new concept, it did not find wide acceptance until the last few years. The utilities have started re-examining the avenues of load management with residential customers. Various methodologies for residential load management are discussed in this section.

\subsubsection{Direct Load Control}

Direct Load Control (DLC) is a traditional way of achieving peak load management. These programs typically involve controllers that allow utilities to turn off or cycle residential appliances for a short time $[1,8]$. Residential central air conditioners, water

\footnotetext{
${ }^{6}$ Load serving entity is a load aggregator or electric distribution utility that purchases power in wholesale and sells it to end-use customers [2]
} 
heaters, swimming pool pumps, and refrigerators are some of the most common residential appliances involved in direct load control.

The utility sends a load curtailment signal to the demand controller connected to the appliances at the customer premises by way of a radio frequency channel, powerline carrier $^{7}$, or private wireless communication network [8]. The residential appliances are directly cycled or turned off by the utility when the power system is constrained with peak demand. Such load curtailment is in effect only at the times of peak demand. The utility operates the load control schedules so as to reduce the peak load. Figure 5 describes the effect of DLC on the load-duration curve. Table 1 summarizes residential DLC programs offered by various utilities in the United States.

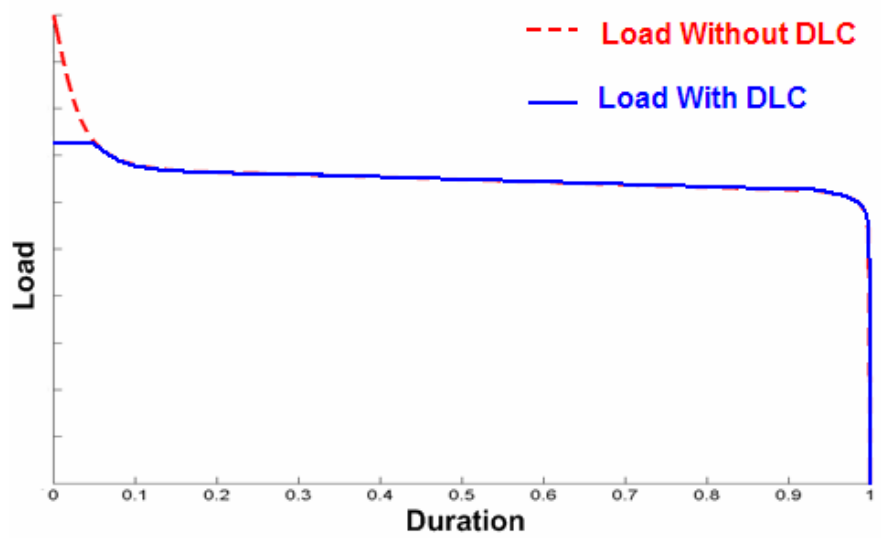

Figure 5: Projected effect of DLC on load-duration curve [2]

Customers participating in DLC programs typically receive monthly credits on their electricity bills, based upon factors such as:

- Type and number of residential appliances under direct control

- Allowed degree of control

- Average amount of load reduction attainable during the curtailment period

- The value of the load reduction to the utility

\footnotetext{
${ }^{7}$ Details of powerline communication or PLC are explained in Section 2.4.1
} 


\begin{tabular}{|l|l|l|}
\hline \multicolumn{1}{|c|}{ Utility Name } & Program Name & \multicolumn{1}{c|}{ Description } \\
\hline $\begin{array}{l}\text { MidAmerican } \\
\text { Energy }\end{array}$ & SummerSaver & $\begin{array}{l}\text { Residential air conditioning cycling program } \\
\text { http://www.midamericanenergy.com/htm//energy3.asp }\end{array}$ \\
\hline Nevada Power & Cool Credit & $\begin{array}{l}\text { Turn off residential air conditioner } \\
\text { http://www.nevadapower.com/conservation/residential/programs/ }\end{array}$ \\
\hline PEPCO & Kilowatchers ${ }^{\circledR}$ & $\begin{array}{l}\text { Cycling program for residential central air conditioner, } \\
\text { heat pump and water heater } \\
\text { http://www.pepco.com/hm kilowatchers.htm }\end{array}$ \\
\hline $\begin{array}{l}\text { Southern } \\
\text { California } \\
\text { Edison }\end{array}$ & $\begin{array}{l}\text { Residential } \\
\text { Summer } \\
\text { Discount Plans }\end{array}$ & $\begin{array}{l}\text { Residential air conditioning cycling program } \\
\text { http://www.sce.com/RebatesandSavings/Residential/SummerDiscountPlan/ }\end{array}$ \\
\hline $\begin{array}{l}\text { Wisconsin } \\
\text { Public Service } \\
\text { Corporation }\end{array}$ & HELP & $\begin{array}{l}\text { Shed or cycling for air conditioner and water heater } \\
\text { http://www.wisconsinpublicservice.com/home/help.asp }\end{array}$ \\
\hline
\end{tabular}

Table 1: Summary of Residential Direct Load Control (DLC) programs

Figure 6 shows the conceptual representation of conventional DLC programs [35]. With conventional DLC programs, the program package fixes the incentives and control strategy, as well as the technology [11]. Using selective load shedding with DLC, the utilities can achieve a significant peak load reduction. However, peak load reduction is achieved at the expense of the customer's comfort and welfare. Utility plays a major role in making load management decisions. Once a customer signs up for the DLC program, he/she has no control over the appliance usage during peak periods. If the DLC service impacts are undesirable, the only choice left for the customer is to withdraw from the program.

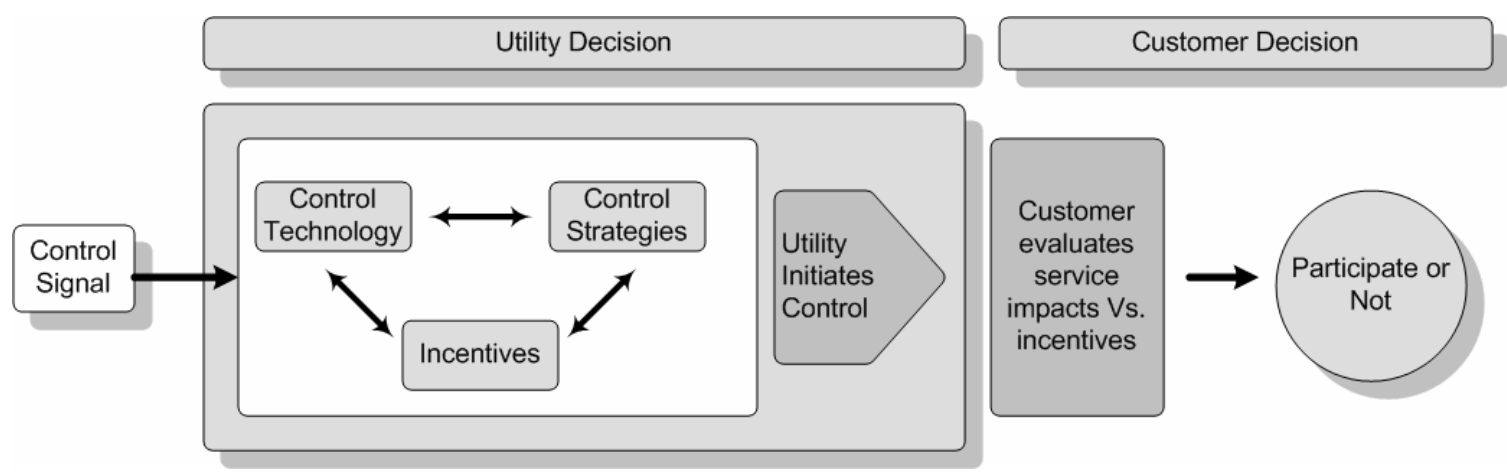

Figure 6: Conventional Direct Load Control 


\subsubsection{Price Responsive Load Management}

As mentioned previously, the generation cost for supplying additional load during the peak period is much higher. Thus, price spikes are associated with peak demands. In regulated power markets, the residential customer is charged by the regulated retail price, which is constant for a few months. With such a flat price structure, the residential customer gains no idea of the status of the power system - whether it is constrained or not. The lack of sufficient information causes the residential demand to be inelastic.

Electricity price can be considered a good indicator of the system condition. In the electricity market, high prices and high price volatility occur concurrently and indicate the risk of system instability. If it can be incorporated in load management programs, the demand elasticity can be increased. When the customer gains the ability to control energy usage, high price hours provide an incentive to reduce the usage. A category of load management programs in which the peak load is controlled in response to the dynamic pricing signals is known as price responsive load management (PRLM). The PRLM programs vary significantly depending on the extent of price volatility passed to the residential customer. If the customer sees varying prices, he/she will make the effort to utilize the part of the day with relatively lower electricity price to the fullest. More residential loads can be operated during these hours instead of during expensive hours. The effect of such load shifting can be projected using the load-duration curve as shown in Figure 7.

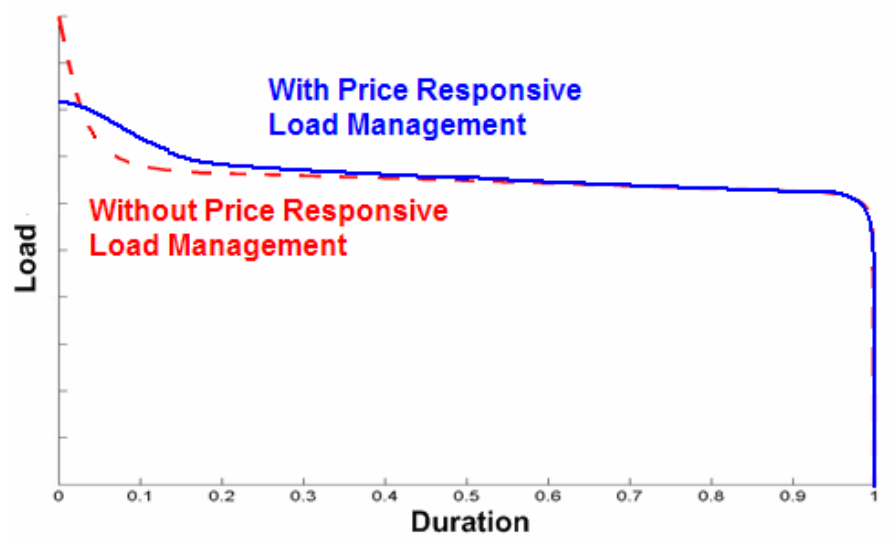

Figure 7: Projected effect of PRLM on load-duration curve 
Figure 8 represents the concept of PRLM programs and customer involvement in making decisions [35]. The customer gains control over decisions regarding control strategy, as well as control initialization. He/she examines service impacts against load shifting incentives to determine how and when to modify demand. Therefore, PRLM programs increase choices for the customer.

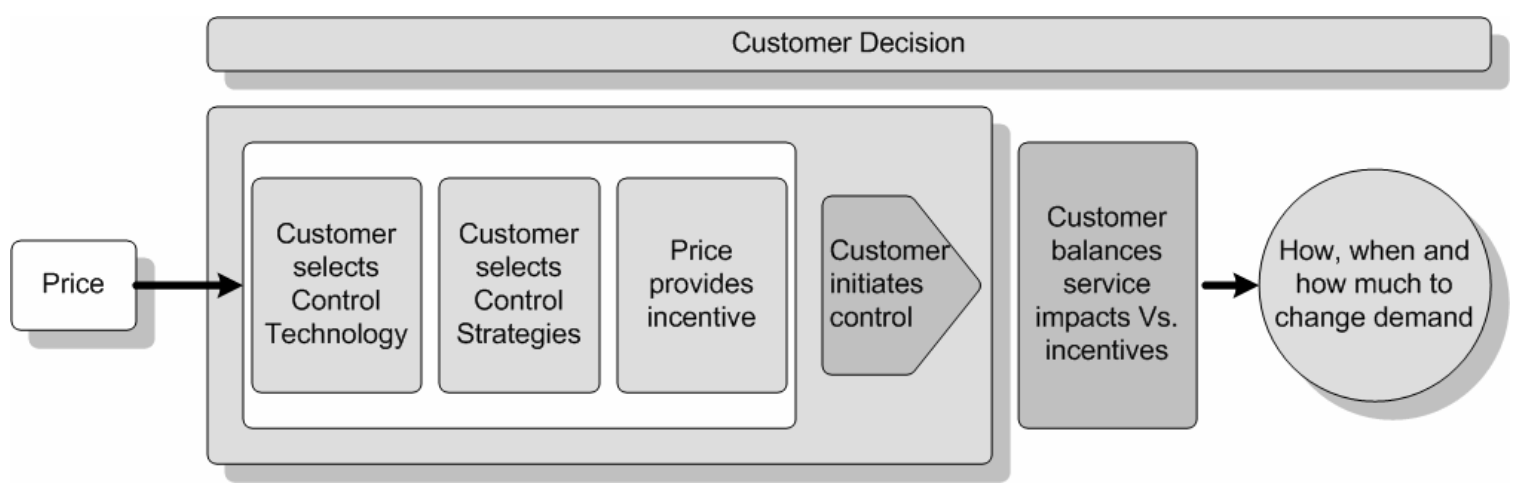

Figure 8: Price Responsive Load Management - Increased Customer Choice

Time of Use (TOU) and Real Time Pricing (RTP) are the two important dynamic pricing strategies in the electricity market in which the customer faces variable electricity charges. These pricing methodologies are explained below.

\subsubsection{Time of Use}

TOU is the simplest form of dynamic pricing, which defines interval-based prices for electricity to reflect utility cost structure. The utility defines on-peak, off-peak, and/or shoulder-peak periods and corresponding prices. The definition of these periods varies with the type of day (weekday or weekend), as well as the season. The customers are charged for their electricity consumption based upon the time of day in which it is used.

The simplicity of both comprehension and use for the customer is the most important advantage of TOU based load management. The customer is aware of the electricity prices throughout the day, which enables him to shift loads to off-peak periods. 
In an effort of peak load management, many utilities have introduced either voluntary or mandatory TOU options. Pacific Gas and Electric Company (PG\&E), Baltimore Gas and Electric Company (BGE), Portland General Electric (PGE), and Alabama Power Company are just a few of the utilities who offer load management programs based on TOU [8].

Figure 9 explains Alabama Power Company's residential TOU Schedule - FDT for the summer period ranging from June 1 through September 30 against the flat rate schedule FD [9].

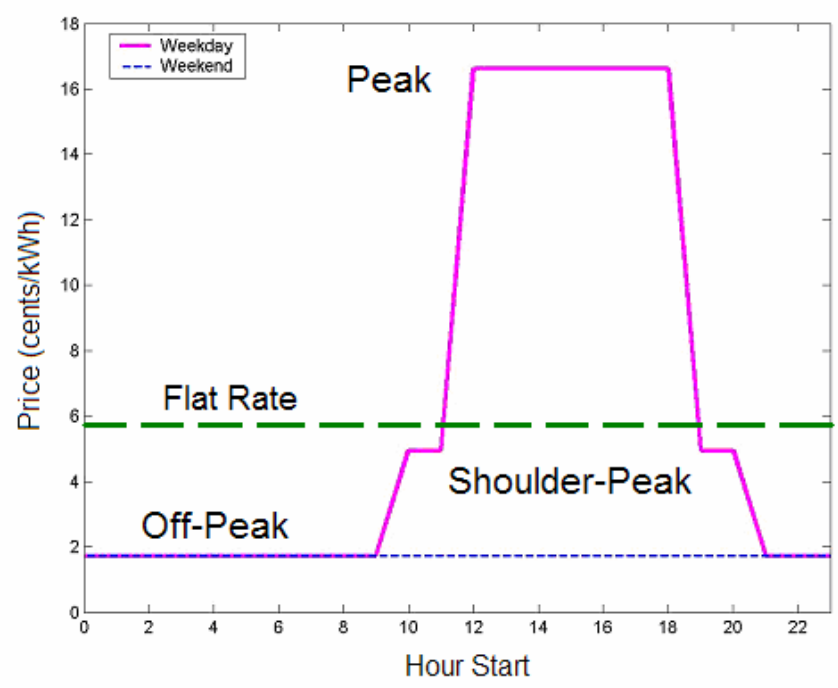

Figure 9: Alabama Power Company's Residential TOU Schedule - FDT [9]

As the TOU price structure is defined well in advance, it lacks the ability to forecast the effects of weather or generation failure on the peak load. Though TOU provides better signals to the customers than current flat rates, it imparts only approximate information of the system condition because of the limited steps in the price variability. Also, in an effort to provide more advanced price notification, TOU prices tend to reflect a less accurate state of supply/demand interaction in the power system [4]. If the technology enables the customers to quickly adjust the demand pattern in response to the price changes, TOU rates fail to send the required information that the customers need to make changes in the demand. 


\subsubsection{Real Time Pricing}

RTP is the most complete expression of dynamic pricing in which customers face a changeable price that varies at a specific time interval. Normally, RTP prices change on an hourly basis. The extent of advance notification of hourly prices differs from market to market. The LSEs can announce hourly prices at the beginning of the hour, or even a day ahead. If the time between the price announcement and execution of price-responsive load control action is increased, the customers can plan their electricity usage schedule in advance. But on the other hand, it will result in a less accurate representation of the actual supply/demand simulation in the market [4]. Thus, there is always a tradeoff between the greater advance price announcements and accuracy of the price signals.

RTP prices reflect variations in wholesale market prices. In RTP schedules, the customers do not necessarily have to be charged at the wholesale price. The wholesale price that the utility sees is obtained by the day-ahead contract and real-time interaction between the generators, independent system operator (ISO), and the utilities [10]. Retail customers are risk adverse and thus, will be unwilling to face the highly varying wholesale prices directly. Real time rates for the retail customers would require some sort of hedging that would alleviate the risk of unstable electricity bills. The implementation of RTP price design and the hedging mechanism is beyond the limits of this thesis. It is assumed that the load control is based on the wholesale real time prices.

Figure 10 shows an example of the wholesale real time prices for the $\mathrm{PJM}^{8}$ market on June 26, 1999.

\footnotetext{
${ }^{8}$ PJM Interconnection, LLC is the organization responsible for the operation and control of the power system in Pennsylvania, New Jersey, Maryland, Virginia, Delaware, West Virginia, and the District of Columbia.[2]
} 


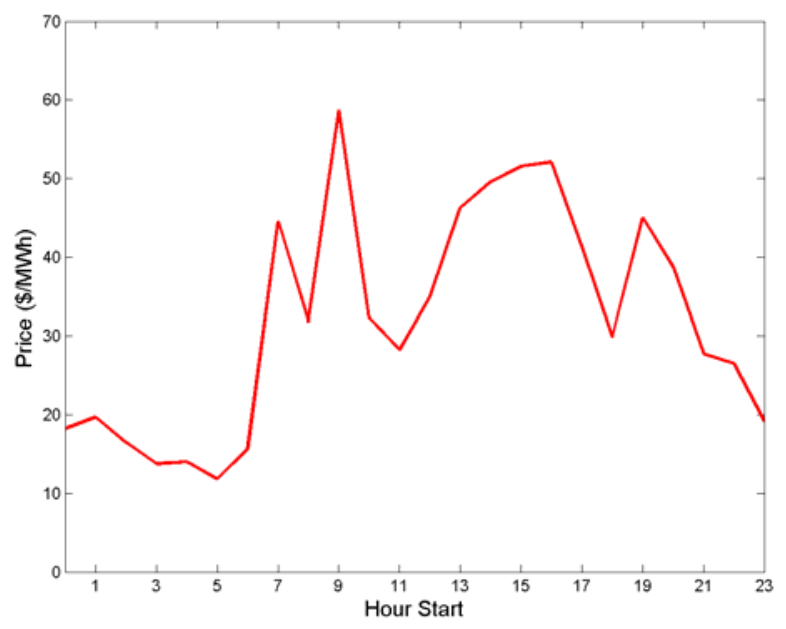

Figure 10: Spot Prices (PJM Market on June 26, 1999)

The RTP programs provide an incentive to the consumer to modify the load profile. Technology plays an important role in the implementation of the load management based upon RTP. It needs infrastructure to communicate hourly price signals to the households. The load control mechanism responsive to RTP signals must be automatic because of the dynamic nature of RTP.

Summarizing the different dynamic pricing structures, we see that all these structures reveal the electricity supply-demand condition with price as an indicator. If the end-users are given the technological ability to control their energy requirements during critical demand period indicated by high price, they can contribute towards peak load management. In the next section, the technologies that enable price responsive load management are discussed in detail.

\subsection{Enabling Technologies}

Smart enabling technologies constitute the backbone of the price responsive load management programs. The actual implementation of the price responsive load management system varies with the pricing strategies. Figure 11 shows an overview of a typical price responsive load management system and communication technologies. 


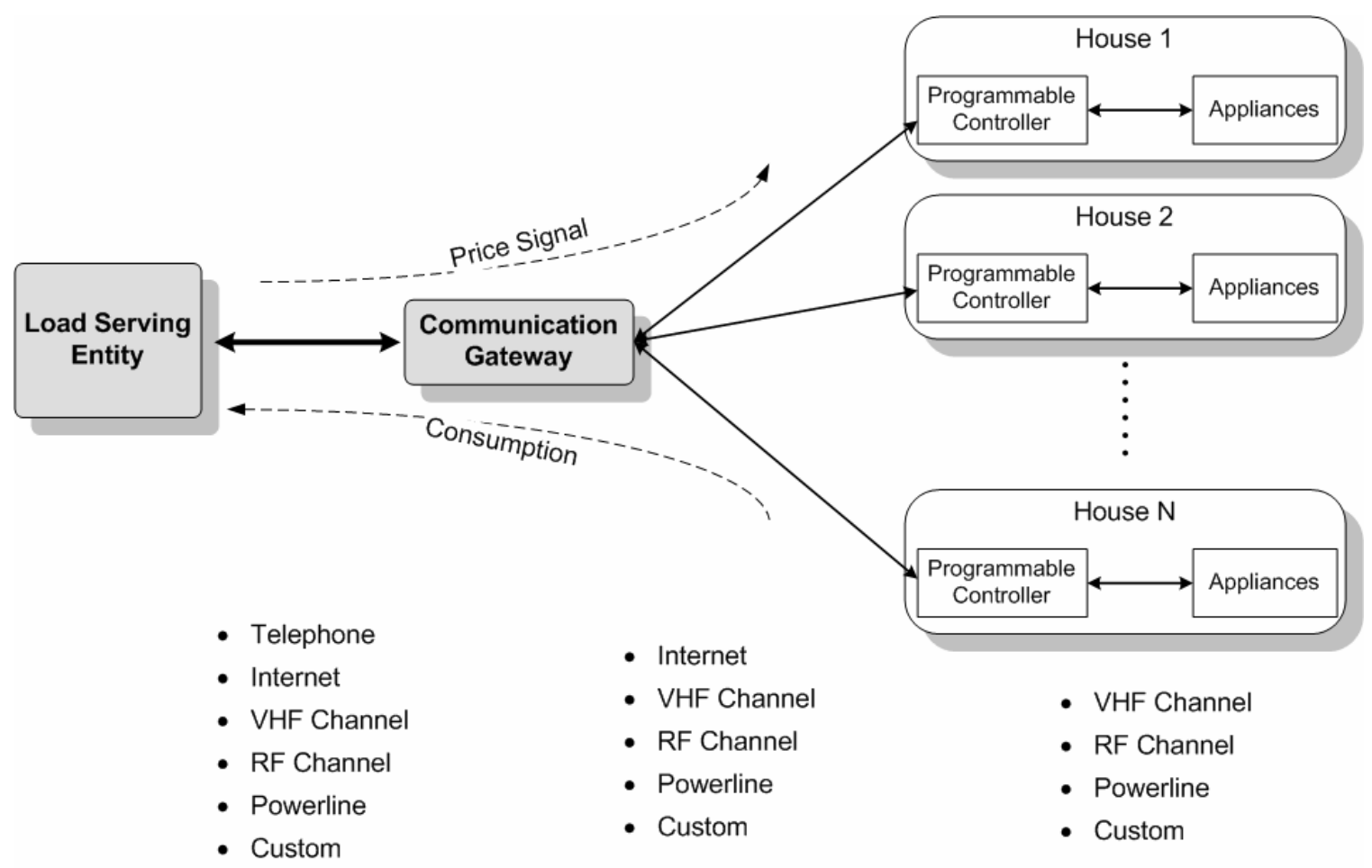

Figure 11: Overview of communication technologies enabling PRLM

There are four basic components of the price responsive load management system [11]:

1. Pricing mechanism

2. A gateway system

3. Load controller and appliance controllers

4. Communication system

\section{Pricing mechanism}

The pricing mechanism is a prime factor for the load controller, which determines the actual implementation of load control. The pricing mechanism defines the retail electricity prices along with incentives or credit structures. The price responsive load management programs are normally based on variants of Time-of-Use and Real Time Pricing explained earlier. 


\section{A gateway system}

A gateway system establishes communication with the energy company or a load serving entity over a telephone line, RF communication link, or a utility-owned network. The gateway system communicates with the load controller over a local communication network. Powerline carrier communications, such as X10, CEBus, and LonWorks, are common communication media for the local control. The gateway system may also incorporate the automatic meter reading facility. Invensys's RF Gateway [12] and Comverge's MainGate [13] are the two well-known gateway systems in the load management market.

\section{Load controller and appliance controllers}

The load controller enables the customer to program the load management system according to his/her comfort requirements and schedule. The load controller is responsible for setting the operation of the appliances under load control. The appliance controllers are essentially the load control switches and controllable thermostats, which are wired into the circuitry of existing home appliances like the air conditioner, water heater, and washer/dryer. The load controller and appliance controllers talk over a local communication network. A wide range of appliance controllers supporting different home networking technologies are readily available.

\section{Communication system}

Developments in communication and home networking technologies have given a boost to the development of residential management systems. The communication between the load serving entity and the residential gateway can range from a standard telephonic connection to the internet. Two leading vendors, Cannon Technology and Comverge, support VHF communication. Powerline carrier and RF communication technologies dominate the home networking market. The next subsection discusses some home networking technologies in detail. 


\subsubsection{State of The Art in Home Networking Technology}

The communication networking for a house is an essential area of study in the development of home energy management systems. It provides centralized linking of the sensors and controllers for the appliances to be controlled by the control panel [14]. There are numerous regional, as well as international, standards to control home appliances; however, the standards based on the Powerline Carrier (PLC), such as X10, dominate the home automation industry. PLC utilizes pre-existing AC electrical networks as transmission medium. It provides exciting home networking solutions as it requires no additional cost or effort associated with the cabling. X10, CEBus, and LonWorks are three dominating protocols for home networking. These protocols are explained briefly in subsequent sections.

\subsubsection{CEBus}

The CEBus viz. Consumer Electronic Bus standard was developed in 1984 by the Electronic Industries Association (EIA) to allow all home appliances to communicate over various media [15]. It was intended for uses such as remote controls, security systems, home entertainment, and for energy management. CEBus is a packet-oriented, connectionless, peer to peer network protocol that supports the Carrier Sense Multiple Access/ Collision Detect Contention Resolution (CSMA/CDCR). It basically follows the ISO/OSI seven-layer network model ${ }^{9}$ specifying the physical, data link, network, transport, session, presentation, and application layers $[15,16]$.

The physical layer of the CEBus is defined by the following four specifications:

1. PLBus (Power Line Bus)

2. SRBus (Single Room Bus or infrared)

3. RFBus (Radio Frequency Bus)

\footnotetext{
${ }^{9}$ The International Standards Organization (ISO) defined the Open System Interconnect (OSI) model as the standard model for networking protocols and distributed applications.
} 
4. WIBus or WIredBus (twisted-pair wires, coaxial cables, and fiber optics collectively)

The physical transmission medium with the other standards will be explained in subsequent subsections, while only the Power Line Bus (PLBus) will be explained here. PLBus uses a $120 \mathrm{kHz}$ carrier to denote superior state, and an absence of carrier signal indicates an inferior state. Though PLBus is the slowest of all other transmission media, it still manages to achieve a data rate of 1000 bits per second with a unit symbol time (UST) of $1 \mathrm{~ms}$. PLBus can transmit a carrier burst at any time, even in the absence of a $60 \mathrm{~Hz}$ power signal, unlike the X10 standard explained later, which transmits a carrier burst only at the $60 \mathrm{~Hz}$ zero crossing. The data link layer is responsible for building the Link Protocol Data Unit (LDPU), which contains the packet type, priority, and privilege along with providing error detection and retransmission. The network layer builds the Network Protocol Data Unit (NPDU), which determines the media that will receive the packets. The application layer builds the Application Protocol Data Unit (APDU) which is used to convey error information and type of service and communication. The Common Application Language, CAL, defined by the EIA, presents a data structure that enables the devices to communicate intelligently with each other.

The CEBus standard incorporates spread spectrum powerline technology. In this technology, a carrier consists of a series of short, self-synchronizing frequency chirps which typically range from 100 to $400 \mathrm{kHz}$ over a duration of $100 \mu \mathrm{s}$. The frequency of the chirp is first swept from 200 to $400 \mathrm{kHz}$ and then from 100 to $200 \mathrm{kHz}$.

Though the CEBus standard seems to be very promising for home automation, the market for CEBus devices has not emerged to the fullest, as in the case of X10.

\subsubsection{LonWorks}

The LonWorks, also known as LonTalk, protocol was developed by Echelon. The protocol offers communication services that allow the application program in a device to 
send and receive messages from other devices over the network without the knowledge of the topology of the network or any information about the other devices [17].

The LonWorks protocol is a layered, packet-based, peer-to-peer communication protocol similar to the CEBus. It comprises of the seven layers of the ISO/OSI model. The LonWorks protocol is media independent, so the physical layer provides medium-specific modulation interfaces and modulation schemes for media such as twisted pair, powerline, radio frequency, coaxial cable, infrared, and fiber optic.

The link layer defines media access, as well as the framing of the data packets, when a source device transmits a data frame and the mechanism for receiving the data frames and detection of the transmission errors. The network layer defines the packet routing and is responsible for the correct delivery of the packets. The transport layer guarantees the reliable delivery of the packets by using an acknowledgement service. The session layer defines an authentication protocol that determines if the sender is authorized to send the message or not. The presentation layer forms a structure for the data exchanged by the lower layers. This is achieved by encoding the messages as network variables, application messages, or foreign frames. The last layer, the application layer, adds the application compatibility to the data exchanged by the lower layers.

The LonWorks system comprises of the following components: neuron chip control processor and transceivers, LonTalk communication protocol, and LonWorks Network Services. A neuron chip processor forms a common physical interface for every LonWorks device. Each neuron chip is identified by a 48 bit unique Neuron ID. The transceiver interfaces the communication port of a neuron chip to the communication media. The LonWorks is considered a more reliable standard because of its acknowledgement and the automatic resend mechanism. 


\subsubsection{X10}

$\mathrm{X} 10$ is the oldest home networking protocol that communicates over the powerline within the house. The X10 transmission standard alone cannot control the appliances directly. The controller units can communicate with appliance modules connected to appliances using X10 protocol. Now, let us have a brief look at X10 transmission theory [18].

$\mathrm{X} 10$ signals are the short RF bursts representing the digital information to be transmitted or received. X10 transmissions are synchronized to the zero-crossing of the AC power signal. The RF bursts are located within $200 \mu$ s of the zeros crossing point. A binary 1 is represented by a 1 millisecond burst of $120 \mathrm{kHz}$ at the zeros crossing point while binary 0 is presented by an absence of $120 \mathrm{kHz}$. The RF bursts are actually transmitted at the zerocrossing point of all three phases in the three phase distribution system. Figure 12 shows the timing relationship of the X10 signal.

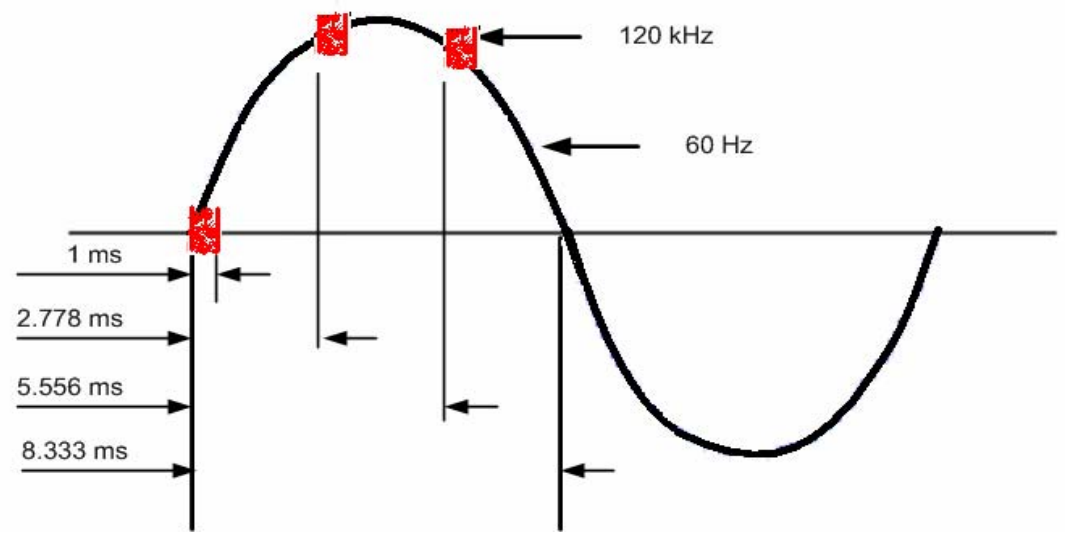

Figure 12: Timing relationship of X10 signals

Each module is recognized by a unique address consisting of a house code (' $\mathrm{A}$ ' to ' $\mathrm{P}$ ') and a device code (1 to 16), making a total of 256 unique addresses possible. One X10 transmission code comprises of eleven cycles of the powerline. Start code, house code, and key code (number code or function code) together form one complete frame of the X10 code. Once a block containing the house code and the number code has been sent twice, the transmission halts for three powerline cycles and then sends a block with the house code and the function code twice. The only exceptions for this rule are dim and 
bright functions in which there is no halt between the codes. Figure 13 shows a frame structure for X10.

\begin{tabular}{|l|c|c|c|c|c|}
\hline $\begin{array}{c}\text { Start } \\
\text { Code }\end{array}$ & $\begin{array}{c}\text { House } \\
\text { Code }\end{array}$ & $\begin{array}{c}\text { Number } \\
\text { Code }\end{array}$ & $\begin{array}{c}\text { Start } \\
\text { Code }\end{array}$ & $\begin{array}{c}\text { House } \\
\text { Code }\end{array}$ & $\begin{array}{c}\text { Number } \\
\text { Code }\end{array}$ \\
\hline \begin{tabular}{|c|c|c|c|c|} 
Start \\
Code
\end{tabular} & $\begin{array}{c}\text { House } \\
\text { Code }\end{array}$ & $\begin{array}{c}\text { Function } \\
\text { Code }\end{array}$ & $\begin{array}{c}\text { Start } \\
\text { Code }\end{array}$ & $\begin{array}{c}\text { House } \\
\text { Code }\end{array}$ & $\begin{array}{c}\text { Function } \\
\text { Code }\end{array}$ \\
\hline
\end{tabular}

Figure 13: X10 Code Transmission

Within each block of data, each individual bit code is transmitted in true, as well as its complement form, on alternate half cycles of the powerline. Figure 14 explains how the bits are sent.

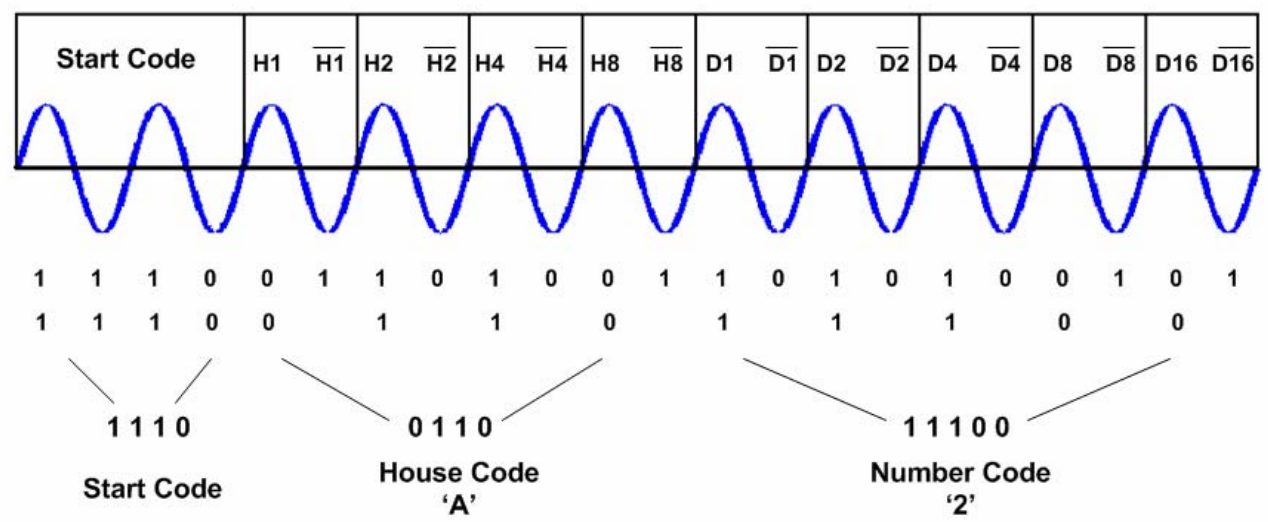

Figure 14: Bit transmission in X10

The X10 home automation standard has been accepted worldwide, and a number of third party vendors have made various X10 devices and interfaces available at very affordable prices.

$\mathrm{X} 10$, CEBus, and LonWorks are the three popular standards in the home automation field. LonWorks provides the most reliable automation solution at the highest cost. Thus, LonWorks systems are particularly suitable for the industrial automation requiring precise and reliable control. The CEBus is relatively less expensive as compared to LonWorks. CEBus incorporates an error correction methodology which makes it fairly 
reliable and error-free. X10 offers affordable home automation at the cost of loss of reliability. The X10 standard is very prone to noise and signal degradation, and thus, it is never $100 \%$ error-free. X10 is also prone to message collisions on the powerline, thus, its usage is limited to the non-critical operations. However, for most home automation applications, it provides a reasonable solution.

The cost effectiveness and ready availability are prime concerns for selecting a standard for the computer aided home energy management system. The home automation system need not be very precise and free of errors as far as the automation is concerned for noncritical operations. X10 devices and controllers are available on the shelf at very affordable prices. Thus, X10 is the obvious choice for the home energy management system.

\subsubsection{Load Controllers}

The operation of appliances can be controlled with the help of load controllers. The load controllers include programmable thermostats, load switches, and dimmers. Many smart appliance controllers are readily available that allow the customer to enter daily, weekly, as well as seasonal, schedules for various device operations. Recently, home networking was integrated with programmable load controllers to enable remote appliance control as well.

Because of the ready availability and our intent to use X10 based load controllers, let us limit our discussion of load controllers to X10 controllers.

The dedicated, as well as computer connected, X10 load controllers are available. Mini/Maxi controllers allow simple on/off/dim control of appliances from a fixed remote location. These dedicated controllers communicate with appliance controllers when plugged into any electric socket. The computerized controllers are connected to the computer through a serial port and to the appliance controllers through the electric outlet. The user can program different schedules and events with the accompanying software. 
Such controllers are more user-friendly, and their functionality can be extended very easily as compared to the dedicated controllers.

The appliance modules form a class of devices that receives X10 signals from load controllers when connected to the electric wiring within the house. Appliance modules offer on/off control of the appliances connected to them. The functionalities of the lamp module include on/off and dim/bright actions. X10 based thermostat controllers offer an easy way to monitor and/or control the room temperature. 


\section{Chapter 3}

\section{Computer Aided Home Energy Management System}

\subsection{Description of CAHEM System}

The Computer Aided Home Energy Management (CAHEM) system enables the implementation of price-responsive load management for the residential sector. As personal computers are rapidly becoming a part of houses in the United States, the use of a personal computer for the prototype CAHEM system is obvious. Eventually, the CAHEM system will be implemented with a dedicated microcontroller system. Figure 15 represents a block diagram for peak load control with the CAHEM system.

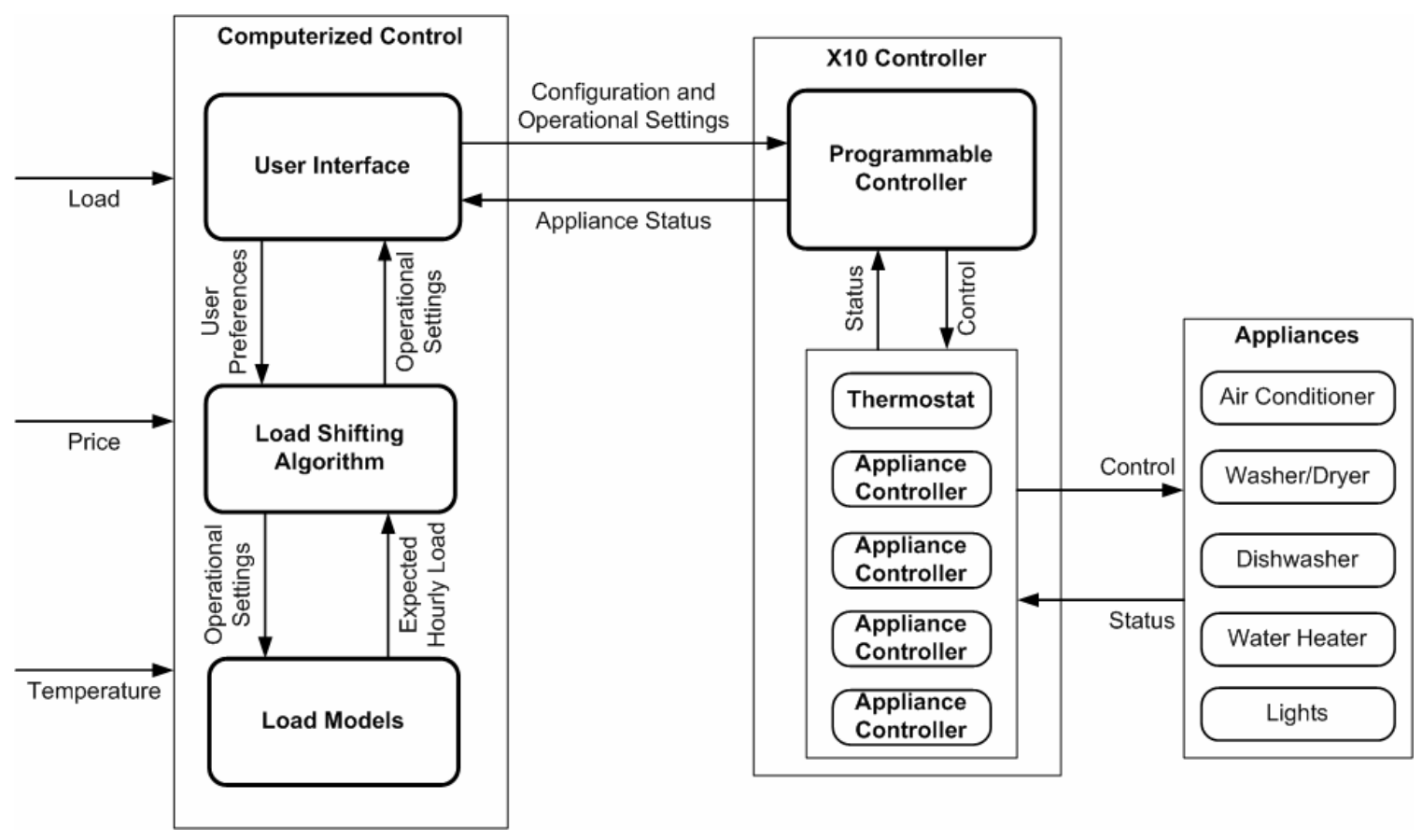

Figure 15: Block diagram for the CAHEM 
The CAHEM system consists of a computerized load control implemented with the help of X10 appliance controllers. A computerized control is composed of the following three units:

1. Load Models: Load models for the residential end-uses calculate hourly electricity consumption in a housing unit. Estimation of the hourly electricity consumption helps in designing optimal load shifting strategies. Load models also enable us to simulate an aggregate effect of peak load management programs.

2. User Interface: A user interface is required for the user to set the operational preferences for various end-uses under price responsive control. The user interface conveys the current level of appliance control to the customer. It also controls load controller hardware.

3. Load Shifting Algorithm: This unit determines load shifting and appliance scheduling based on the price, load, and temperature data, along with customer preferences. It decides operational settings for residential appliances, e.g. thermostat setting of a central air conditioner.

Home automation hardware enables actual control of the appliances. It consists of a programmable controller which interfaces to a personal computer and incorporates a twoway transmission of powerline signals. A controller sends control signals over existing electric wiring to the receiver modules dedicated to each appliance. The receiver modules control the appliances depending on the control command received from the main controller. The user can request the status of each appliance connected to the programmable controller with the help of the user interface.

\subsection{Residential End-Uses}

Average urban houses in the United States are equipped with a wide range of electricity end-use appliances. Typical residential end-uses of electricity include air conditioning, 
water heater, lights, cooking appliances, refrigerator, dishwasher, washer, and dryer. Figure 16 shows the breakdown of energy consumption by the end-use in the average American home [19].

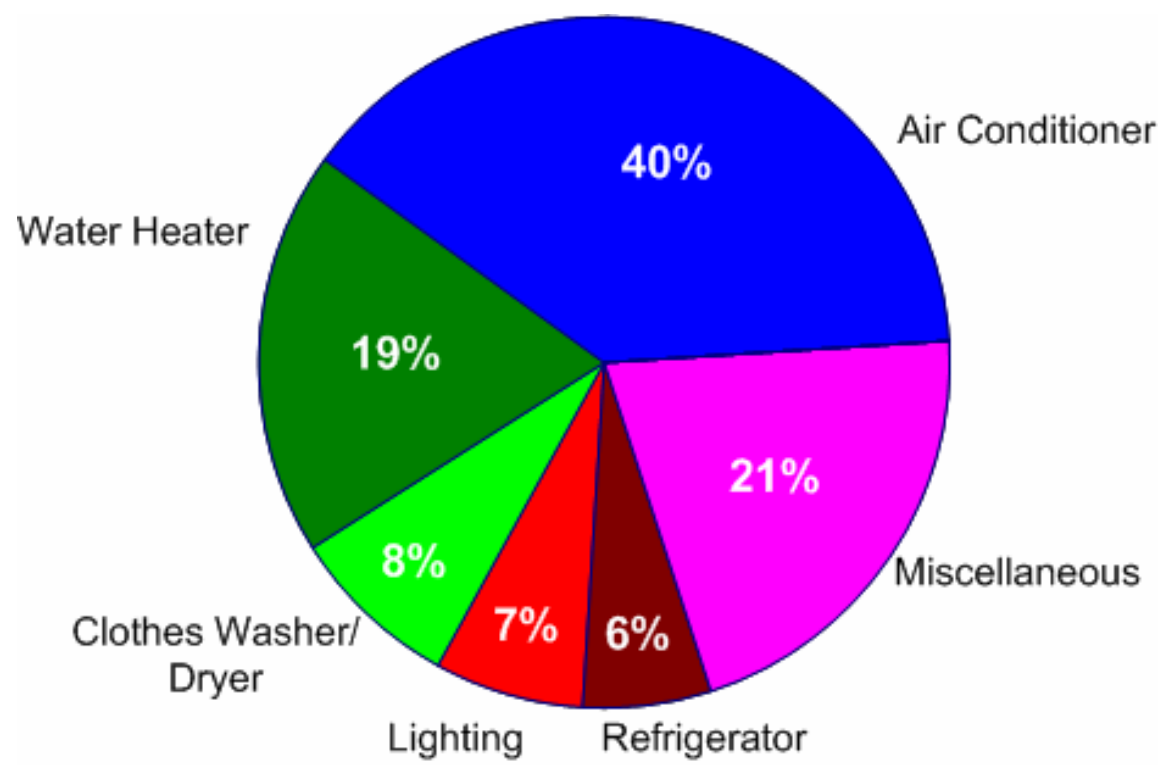

Figure 16: Breakdown of residential electricity consumption

\subsubsection{Categories of End-Use Devices}

Schweppe and Daryanian [20] state that an end-use device 'uses' electricity to provide a 'service' to the consumer. In price responsive load control, the demand from the residential end-use devices can be altered by modifying the device's usage and/or the service it provides. The residential end-use devices can be classified into three basic categories depending on load control methodology. Figure 17 summarizes the categories of residential end-use devices.

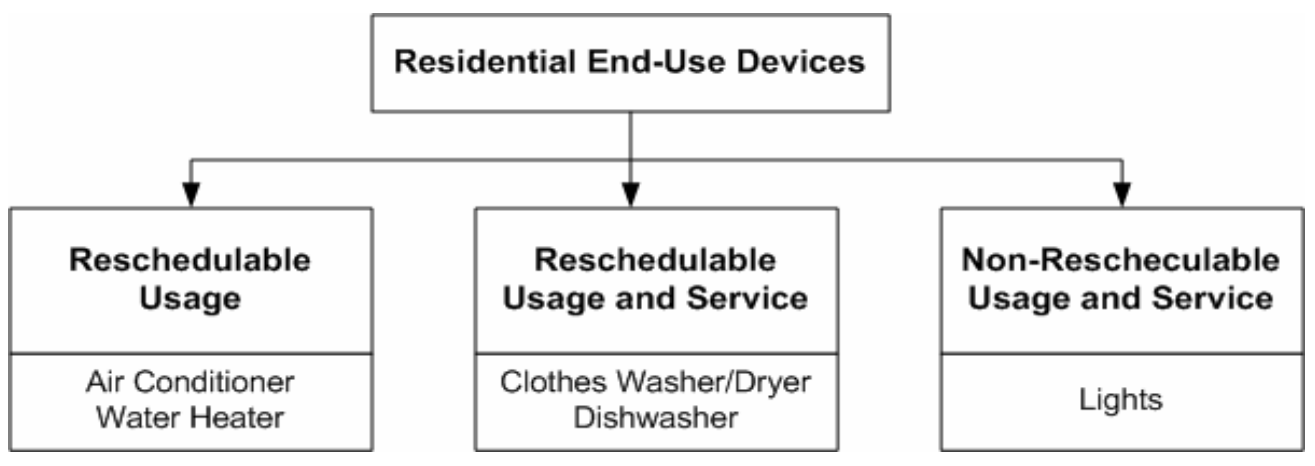

Figure 17: Categories of residential end-use devices 
1. Reschedulable usage: This category of end-use devices makes use of a property of thermal storage that allows rescheduling of usage. Air conditioners and space heaters fall under this category. In the case of air conditioners, thermal storage or thermal inertia helps in modifying times at which electricity is being consumed, maintaining reasonable comfort for the consumer.

2. Reschedulable usage and service: The residential end-use appliances in this category can be operated at different times to get service. Common examples of reschedulable appliances are dishwasher, clothes washer/dryer, and other cleaning appliances.

3. Non-reschedulable usage and service: This category is characterized by nonreschedulable loads that can only reduce usage and service, like lights. The usage of such loads can be reduced, which in turn reduces the level of service provided by these loads.

\subsubsection{Load Models}

Development of a fairly accurate representation of the residential hourly loads is one of the most critical steps in assessing the load management programs [21]. The load models for each appliance under load management control helps to calculate the total hourly load for a house. Among all residential appliances, air conditioning contributes significantly to the residential load during the summer, thus offering a great potential for controlling total residential electricity demand [22]. In this section, residential load models are discussed with the air conditioning load model in detail.

\section{Residential Air Conditioner}

The aim of development of the air conditioning load model is to calculate the hourly cooling load for given weather conditions and specified house structure. 'Cooling Load' is the thermal energy that must be removed from the interior of a house in order to 
maintain the desired comfort conditions, normally specified by the interior dry-bulb temperature.

The air conditioning load is controlled by a thermostat determining interior dry-bulb temperature. As inside dry-bulb temperature changes in effort to manage the cooling load, the load model must support hourly load calculations with a variable thermostat setting. Various methodologies for residential air conditioner load calculations, based on statistical analysis of historic usage data, can be found in the literature [22, 23]. However, these models can not be used in the analysis of the price responsive load control strategy because of lack of historic usage data reflecting effect of the variable thermostat setting. Thus, the physically-based model of an air conditioned house has been used in this study.

The heat flow and thermal storage in a house is a very complex process involving a number of parameters, such as temperature, solar radiation, humidity, air infiltration, wind, etc. [22]. The model would be highly complex and intractable with inclusion of all individual parameters and heat transfer processes. Thus, the following assumptions are made to obtain a simple yet fairly accurate model.

1. An air conditioned house is treated as a single-family, detached, single floored house with four walls and a roof exposed to exterior conditions, such as solar radiation and uniform outside temperature.

2. In reality, air conditioned space is divided into a number of zones each with different parameters and thermal properties. However, such zoning is generally unnecessary for a simple house, as air conditioned volume is relatively small. Thus, in our study, an air conditioned house is treated as a single zone with a uniform temperature as a result of sufficient air circulation.

3. There is no independent thermal storage coupled to the main air conditioning system; thus, cooling load calculations conform to the modeling of an air conditioned house with single equipment.

4. The control of only inside temperature is possible with the thermostat controller. There is no available control of humidity. Thus, the effect of humidity control is not considered. 
5. Construction material for each component of a house envelope, such as walls, roof, and windows is uniform for a house. (e.g. all walls for a house will have the same construction material)

6. Average power densities for lights in a house are around 3 to $5 \mathrm{~W} / \mathrm{m}^{2}$ [23]. Heat gain due to lighting is ignored in cooling load calculations, as its contribution to heat gain is insignificant.

The dynamic behavior of an air conditioned house is modeled on the basis of underlying physical principles, such as the thermodynamics of the house and the effect of external, as well as internal, conditions affecting heat transfer across the house envelope [22]. Thermal networks can be used for the dynamic modeling of an air conditioned house. The model used in our study is a lumped parameter thermal network, shown in Figure 18, representing the dynamics of heat flow and thermal properties of an air conditioned house described in a text by Kreider and Rabl [25].

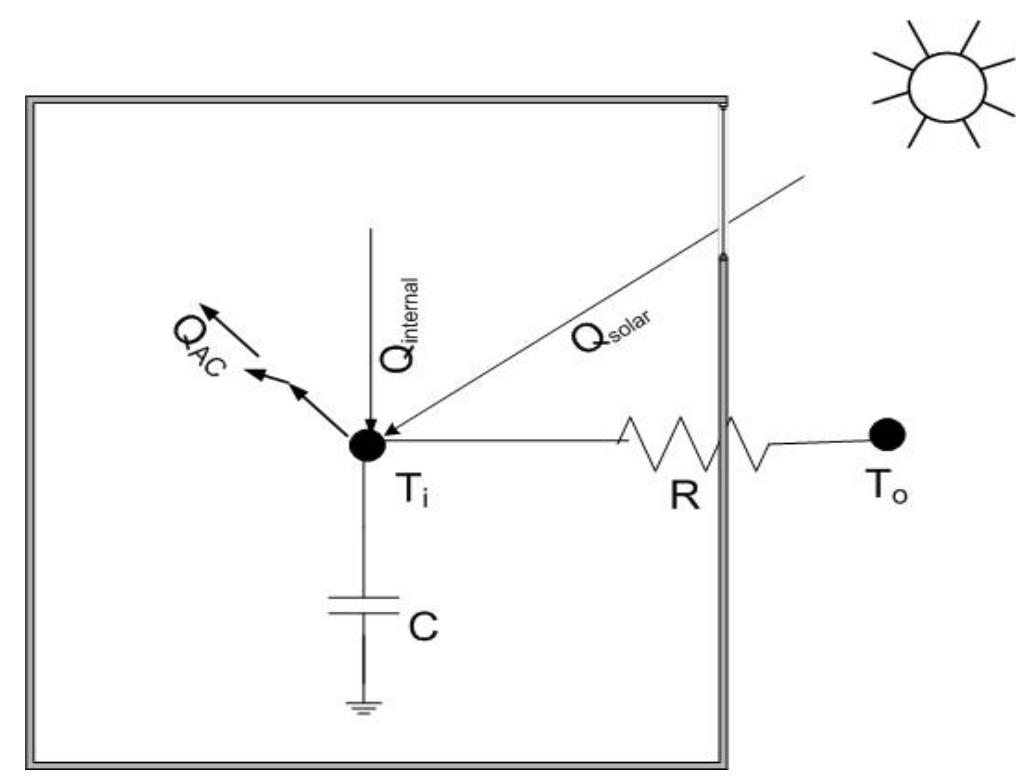

Figure 18: Thermal network model for air conditioned house [22, 25, 26]

The heat flow across the house envelope is represented by resistance (R). Normally, the building envelope consists of the walls, roof, and windows, contributing to a conductive heat flow. Such heat flow due to each component of the envelope can be obtained by 
multiplying the conductance of the construction material (U), its surface area (A), and the temperature difference across the envelope.

The total conductive heat flow from the interior to the exterior of a house at $\mathrm{k}^{\text {th }}$ hour of a day is given by Equation 1:

$$
\dot{Q}_{\text {cond }}=\sum_{m} U_{m} A_{m}\left(T_{i}-T_{o}\right)
$$

The flow of sensible heat, due to air exchange, also contributes to heat flow across the envelope. It is defined as:

$$
\dot{Q}_{a i r}=\rho c_{p} \dot{V}\left(T_{i}-T_{o}\right)
$$

Where, $\rho$ is the density of air, $c_{p}$ is the specific heat of air, and $\dot{V}$ is the air exchange rate in $\mathrm{ft}^{3} / \mathrm{h}$.

The total heat transmission coefficient $K_{\text {tot }}$ includes the total conductive heat transmission coefficient and the air exchange term $\rho c_{p} \dot{V}$.

$$
K_{\text {tot }}=K_{\text {cond }}+\rho c_{p} \dot{V}
$$

Where, the total conductive heat transmission coefficient $K_{\text {cond }}$ is the summation of UA over all surfaces.

$$
K_{\text {cond }}=\sum_{m} U_{m} A_{m}
$$

Resistance $\mathrm{R}$ is the inverse of the total heat transmission coefficient $K_{\text {tot }}$ of a house.

$$
R=\frac{1}{K_{\text {tot }}}
$$

To model thermal inertia, a thermal network must incorporate at least one capacitor (C) representing the heat capacity of a house. The heat balance equation for a thermal network is a first-order differential equation in a variable $T_{i}$,

$$
C \dot{T}_{i}=\frac{\left(T_{o}-T_{i}\right)}{R}+\dot{Q}
$$

The value of $\mathrm{C}$ is based on the effective diurnal heat capacity of a house. The effective diurnal heat capacity is roughly $40 \%$ to $80 \%$ of the static heat capacity of a house. The 
static heat capacity is the summation of the heat capacity of all masses, including air, calculated by multiplying volume, density, and specific heat of each component of the structure.

$$
C_{\text {Static }}=\sum_{m}\left(V \rho c_{p}\right)_{m}
$$

The total heat flow $\dot{Q}$ is a net heat flow; as a result of heat removed by air conditioning equipment, heat gains due to internal sources and solar radiation.

$$
\dot{Q}=\dot{Q}_{A C}-\left(\dot{Q}_{\text {internal }}+\dot{Q}_{\text {solar }}\right)
$$

For convenience, a quantity Time Constant $(\tau)$ is defined, which sets the time scale for cooling/heating.

$$
\tau=R C
$$

The higher the time constant value of a house, the longer it takes to cool down or warm up. Equation 10 presents a solution of a heat balance equation (Equation 6) with timevarying driving terms $\dot{Q}$ and $T_{\text {o }}$.

$$
T_{i}(t)=\exp \left(\frac{t_{0}-t}{\tau}\right) T_{i}\left(t_{0}\right)+\int_{t_{o}}^{t} \exp \left(\frac{t^{\prime}-t}{\tau}\right) \frac{\left[T_{o}\left(t^{\prime}\right)+R \dot{Q}\left(t^{\prime}\right)\right]}{\tau} d t^{\prime}
$$

If the calculations are restricted to an hourly basis, $\left(t-t_{0}\right)$ equals 1 . Let us consider heat flow, $\dot{Q}$ and outside temperature, $T_{o}$ constant for the hour.

$$
\begin{aligned}
& T_{i}\left(t_{o}+1\right)=\exp \left(\frac{-1}{\tau}\right) T_{i}\left(t_{o}\right)+\frac{\left[T_{o}+R \dot{Q}\right]}{\tau} \int_{t_{o}}^{t_{o}+1} \exp \left(\frac{t^{\prime}-\left(t_{o}+1\right)}{\tau}\right) d t^{\prime} \\
& T_{i}\left(t_{o}+1\right)=\exp \left(\frac{-1}{\tau}\right) T_{i}\left(t_{o}\right)+\left(T_{o}+R \dot{Q}\right)\left[1-\exp \left(\frac{-1}{\tau}\right)\right]
\end{aligned}
$$

Let $\varepsilon=\exp \left(\frac{-1}{\tau}\right)$

$$
\begin{gathered}
T_{i}\left(t_{o}+1\right)=\varepsilon T_{i}\left(t_{o}\right)+\left(T_{o}+R \dot{Q}\right)[1-\varepsilon] \quad\left({ }^{\circ} F\right) \\
\dot{Q}_{A C}=\frac{1}{R}\left[\frac{T_{i}\left(t_{o}+1\right)-\varepsilon T_{i}\left(t_{o}\right)}{(1-\varepsilon)}-T_{o}\right]+\left(\dot{Q}_{\text {internal }}+\dot{Q}_{\text {solar }}\right) \quad(\text { Btu } / h)
\end{gathered}
$$


$\dot{Q}_{\text {internal }}$ is heat gained from internal sources, such as occupants and appliances. Heat gain from occupants is in the range of 340 to $850 \mathrm{Btu} / \mathrm{h}$ per occupant, depending upon the physical activities. Residential appliances, such as the television set, refrigerator, computer, etc., cumulatively cause heat gain in the range of 500 to $2,500 \mathrm{Btu} / \mathrm{h}$.

Instantaneous solar heat gain through glazing is dependent upon the window area, shading coefficient (SC), the solar heat gain factor (SHGF), and the cooling load factor (CLF) of glazing defined by the American Society of Heating, Refrigeration and AirConditioning Engineers (ASHRAE).

$$
\dot{Q}_{\text {solar }}=A \times S C \times S H G F \times C L F(B t u / h)
$$

Actual power consumption by air conditioning equipment is dependent upon the cooling load $\dot{Q}_{A C}$, as well as an efficiency of the equipment. The cooling efficiency is normally expressed in terms of Energy Efficiency Ratio (EER), defined as a ratio of heating capacity $(\mathrm{Btu} / \mathrm{h})$ to electric input rate (watts). The power consumption of an air conditioner for an hour under consideration is calculated as

$$
D_{A C}=\frac{\dot{Q}_{A C}}{E E R}(W)
$$

As it is clear from the above description, the fundamental parameters in the model are cooling capacity of air conditioning equipment, thermostat setpoint, ambient weather conditions, and thermal properties of a house [26]. In order to facilitate the use of the residential air conditioner model at an aggregate level study, these parameters are set using random variables with uniform or Gaussian distributions within expected ranges.

In continuation with the description of HVAC model, let us focus on the concept of thermal comfort. The thermal comfort is defined by the ASHRAE as the condition of mind which expresses satisfaction with the thermal condition of an environment. The thermal comfort is subjective in nature, and it is dependent on a number of environmental factors, including air temperature, air speed, and humidity, as well as individual factors 
like level of activity and clothing. It is represented with the psychometric chart, which relates dry-bulb temperature and relative humidity, as shown in Figure 19.

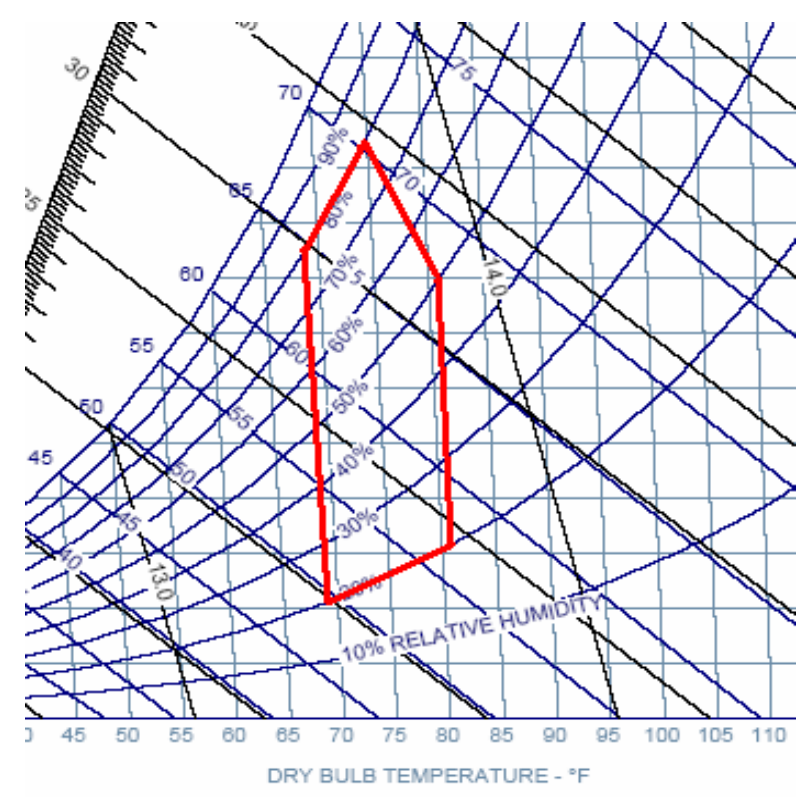

Figure 19: Thermal comfort zone (Courtesy: www.linric.com) [34]

As the effect of humidity is not taken into account while developing the load model for air conditioners, the humidity dimension of the psychometric chart will be neglected. Thus, the thermal comfort zone for human beings ranges from $68^{\circ} \mathrm{F}$ to $81^{\circ} \mathrm{F}$ [33]. The CAHEM load controller will limit its operation to this comfort zone as much as possible.

\section{Water Heater}

The water heater load is the next important residential load. The power consumption of a water heater can be controlled by modifying the duty cycle based on either thermostatic control or timer control. Modeling hourly power consumption of the water heater with timer control is more obvious, and thus, a water heater with timer control is assumed. It is assumed that the hot water usage profile does not vary with price responsive load control. Thus, the water heater is cycled during the peak demand period. 
Water heating energy consumption calculations are used based upon the model described by Pacific Gas and Electric (PG\&E) company [27, 28]. PG\&E found that hot water consumption varies with the conditioned floor area of a house as described in Equation 17.

$$
G P D=24+0.016 \times C F A
$$

GPD $=$ Average daily hot water usage (gallons/day)

$\mathrm{CFA}=$ Conditioned floor area $\left(\mathrm{ft}^{2}\right)$

The hourly hot water usage schedule is expressed as a fraction of the average daily hot water usage (GPD). Figure 20 shows the hot water consumption schedule for an average weekday and weekends separately. Hourly hot water consumption during the hour under consideration (GPH) is obtained by multiplying the average daily hot water usage (GPD) by a fraction specified in Figure 20.

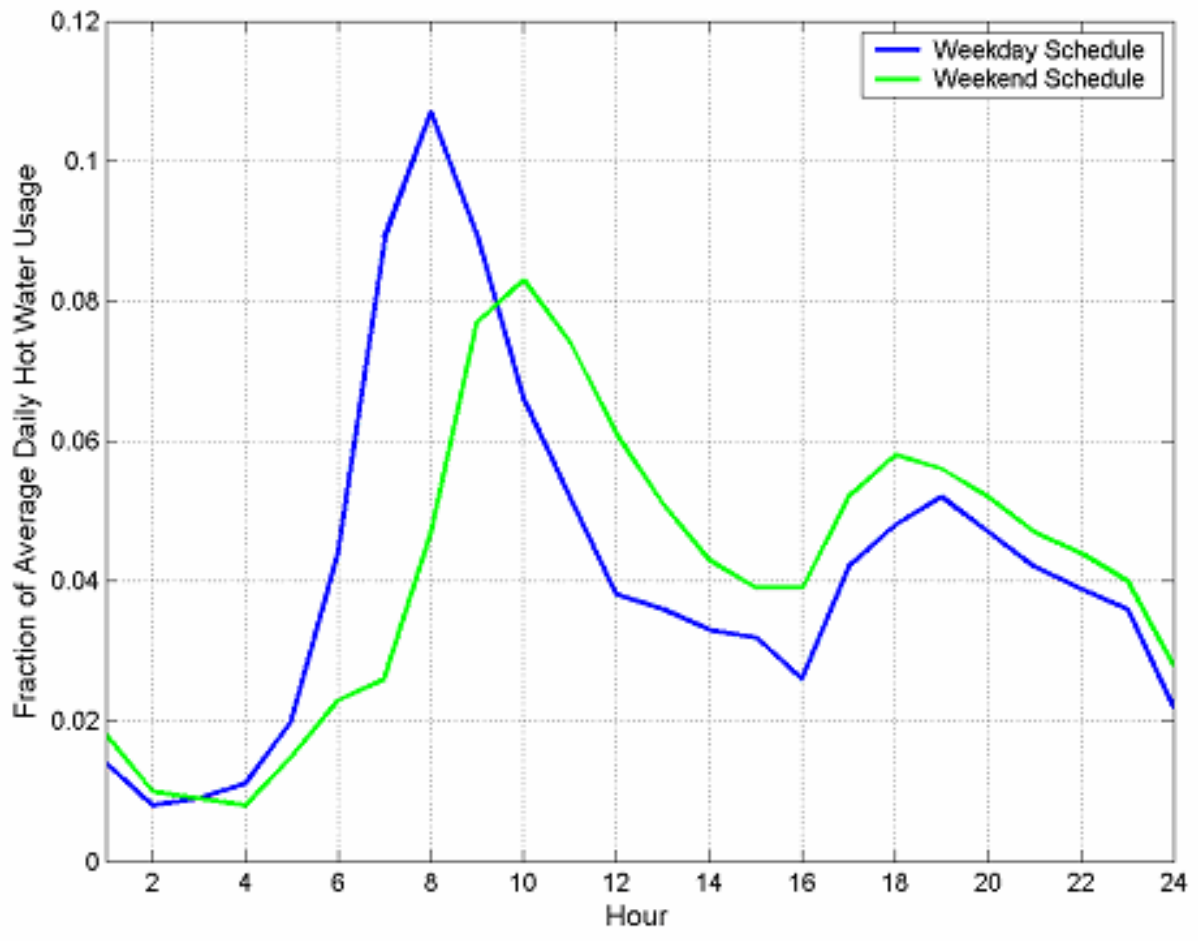

Figure 20: Hourly hot water usage schedule [27] 
The difference between inlet water temperature and hot water temperature is assumed constant to be equal to $65^{\circ} \mathrm{F}$, assuming inlet water temperature at $55^{\circ} \mathrm{F}$ and a constant supply of hot water at $120^{\circ} \mathrm{F}$ [27]. An efficiency of the water heater is normally defined in terms of energy factor (EF). Higher EF means a more efficient water heater. The EF values for electric resistance water heaters range from 0.7 to 0.95 .

In a water heater, as hot water is drawn, the same volume of cold water from the inlet enters. The hourly water heating load (in Btu) is the volume of hot water drawn during the given hour (in gallons) times the difference between inlet water temperature and hot water supply temperature, $\Delta T\left({ }^{\circ} \mathrm{F}\right)$ times heat required to heat a gallon of water by $1^{\circ} \mathrm{F}$. Based on density and the specific heat of water, 8.3 Btu heat must be added to one gallon of water to increase the water temperature by $1^{\circ} \mathrm{F}$.

$$
\dot{Q}_{D W H}=\frac{8.3 \times G P H \times \Delta T}{3.412 \times E F} \quad(W)
$$

Equation 18 gives the hourly water heating load, assuming that the water heater is perfectly insulated; thus, the effect of air temperature on the water heating load can be neglected.

\section{Washer and Dryer}

The washer/dryer is one of the reschedulable loads whose energy consumption is dependent upon the size of the washing load and the temperature of the water used for washing. About ninety percent of the energy consumed in operating a washing machine is used for heating the water during washing and rinsing cycles. Thus, energy consumption per washing load varies with quantity of water required for washing and rinsing. Typical energy consumption for washing and rinsing cycles for commercially available washing machines ranges from $0.5 \mathrm{kWh} /$ cycle to $1.2 \mathrm{kWh} /$ cycle. Clothes drying consume much more energy than clothes washing. The average energy consumption for the domestic dryer is about 2.5 to $3.5 \mathrm{kWh} /$ cycle [29]. 


\section{Dishwasher}

The dishwasher is another common reschedulable load in residences. Energy consumption per cycle for a dishwasher is composed of the machine energy, water heating energy, and drying energy. About $80 \%$ of the energy a dishwasher uses is for heating the water. Typical energy consumption for a residential dishwasher is in the range of 1.2 to $2.4 \mathrm{~kW} /$ cycle, depending on the type of drying. The duration for each dishwashing cycle typically ranges from 50 to 80 minutes, including drying.

\section{Lights}

The annual electricity demand due to interior lighting is related to the furnished floor area $\left(\right.$ FFA in $\mathrm{ft}^{2}$ ) of a house [32], as in Equation 19.

$$
\dot{\mathrm{Q}}_{\text {Lights (Annual) }}=(\text { FFA*0.8 }+445) \mathrm{kWh} / \text { year }
$$

Daily lighting electricity consumption is assumed constant and obtained by dividing the annual load by 365 . Figure 21 shows an hourly lighting usage profile [31]. The actual hourly electricity consumption is calculated by multiplying the daily consumption by the corresponding fraction from Figure 21.

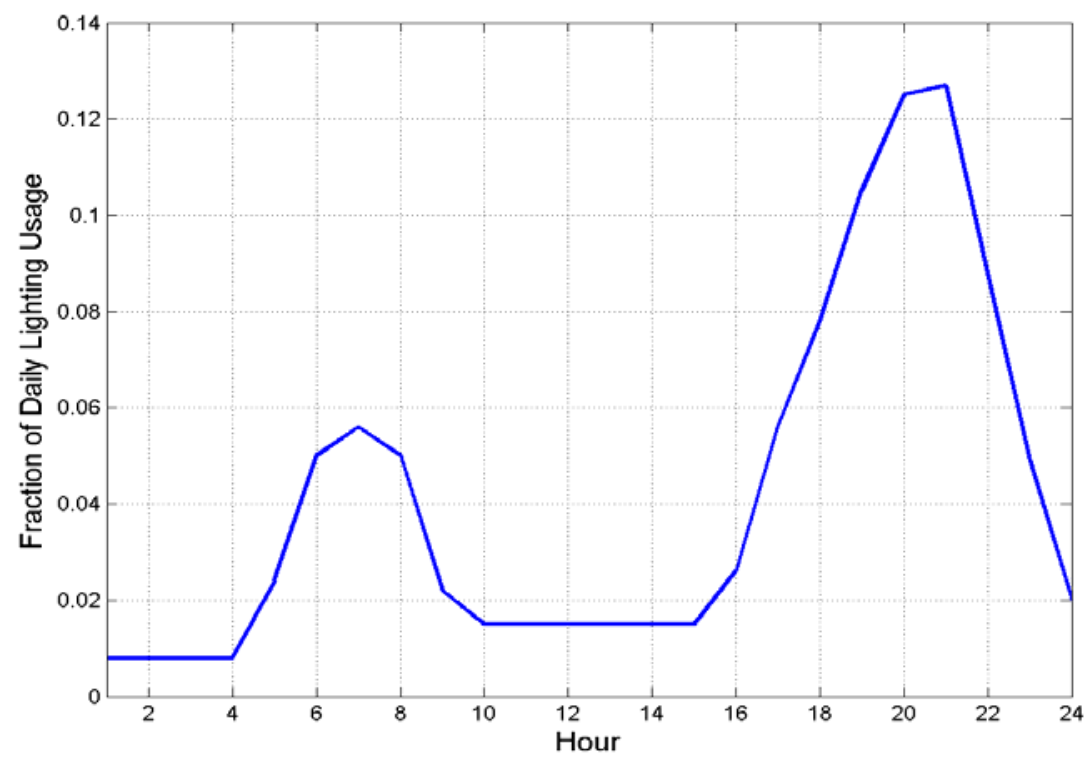

Figure 21: Hourly lighting usage profile [30] 


\subsection{User Interface}

The graphic user interface for the CAHEM system incorporates the following functionalities:

1. Enables the customer to enter his/her choices for controller operation

2. Updates the customer about appliances settings and demand profile

3. Communicates with the hardware controllers for appliances

\subsubsection{Hardware Components}

The CAHEM user interface is demonstrated with the following hardware components:

1. $\underline{X 10}$ Controller (CM11): CM11 is a bidirectional X10 controller that connects to a serial port and converts human readable commands into Interface Communication Protocol. It communicates with the X10 modules to control their operation.

2. Lamp Module (LM465): The lamp module supports on/off and dim/bright control of a lamp connected to it. It communicates with the X10 controller (CM11).

3. Appliance Module (HD243): This heavy duty appliance module is used for controlling water heaters, washers, and dryers.

4. Programmable Thermostat $\left(\right.$ TempLinc $\left.^{\mathrm{TM}}\right)$ : The TempLinc module allows control of the central air conditioner. The X10 controller configures, as well as controls, the operation of the TempLinc unit.

\subsubsection{User Interface Software}

The customer sets preferences depending on his/her priorities - either comfort or peak load reduction benefits. Comfort and peak load reduction benefits are two counteracting factors, meaning the maximization of one factor is equivalent to the minimization of the other. For offering the customer simplicity in control, three modes which characterize different priorities are defined.

1. Super-Save: As is obvious from its name, in this mode the customer chooses to offer more allowance for load control at the cost of reduced comfort. 
2. Economy: This mode tries to balance comfort and benefits for load control. It has narrower tolerances for operational settings than the Super-Save mode.

3. Comfort: It sets a very strict tolerance on the operational settings. The controller will try to maintain the appliances at the desired settings as best as possible.

The operational settings for each mode may differ from system to system. However, in order to simplify aggregate-level simulations, three different sets of settings are specified. Table 2 summarizes the settings for the various modes. Each set of customer preferences includes the following parameters:

1. Price threshold: The load reduction mechanism activates when the price exceeds the price threshold. The higher the price threshold, the lower the incidences of load reduction operation. The setpoints for various modes are chosen in order to keep consistency with $\mathrm{Oh}[5]$.

2. Temperature tolerance: The allowable swing in thermostat settings for air conditioners decides the perseverance of maintaining the desired thermal comfort. This setting is independent of the thermostat setting.

3. Minimum brightness: The lesser the value of minimum brightness, the higher the load reduction that can be achieved.

4. Minimum water heater performance: The water heater performance is the fraction of energy consumption for the water heater at desired settings. The effective water temperature is proportional to the energy consumption (assuming constant efficiency).

5. Maximum delay for washer/dryer and dishwasher: The number of hours between the scheduled operation and deferred operation is defined by the maximum delay.

\begin{tabular}{|l|c|c|c|}
\hline \multirow{2}{*}{ Parameter } & \multicolumn{3}{|c|}{ Setpoint } \\
\cline { 2 - 4 } & Super-Save & Economy & Comfort \\
\hline Price Threshold $(\$ / \mathrm{MWh})$ & 100 & 300 & 500 \\
\hline Temperature Tolerance $\left({ }^{\circ} \mathrm{F}\right)$ & 6 & 4 & 2 \\
\hline Min. Brightness $(\%)$ & 50 & 60 & 70 \\
\hline Min. Water Heater Performance $(\%)$ & 75 & 80 & 85 \\
\hline
\end{tabular}

Table 2: Summary of modes of the CAHEM 
The CAHEM user interface software is built on the MATLAB environment (Version 6.5 R13). It requires the Fuzzy Logic Toolbox installed with MATLAB. Different steps in the configuration and the operation of the CAHEM user interface are as follows.

\section{Step 1: Select Time Scale}

This software will be used for simulation purposes. Therefore, it offers two options for the time scale, as shown in Figure 22. The software will run in either run-time or simulated time when the appropriate option is selected.

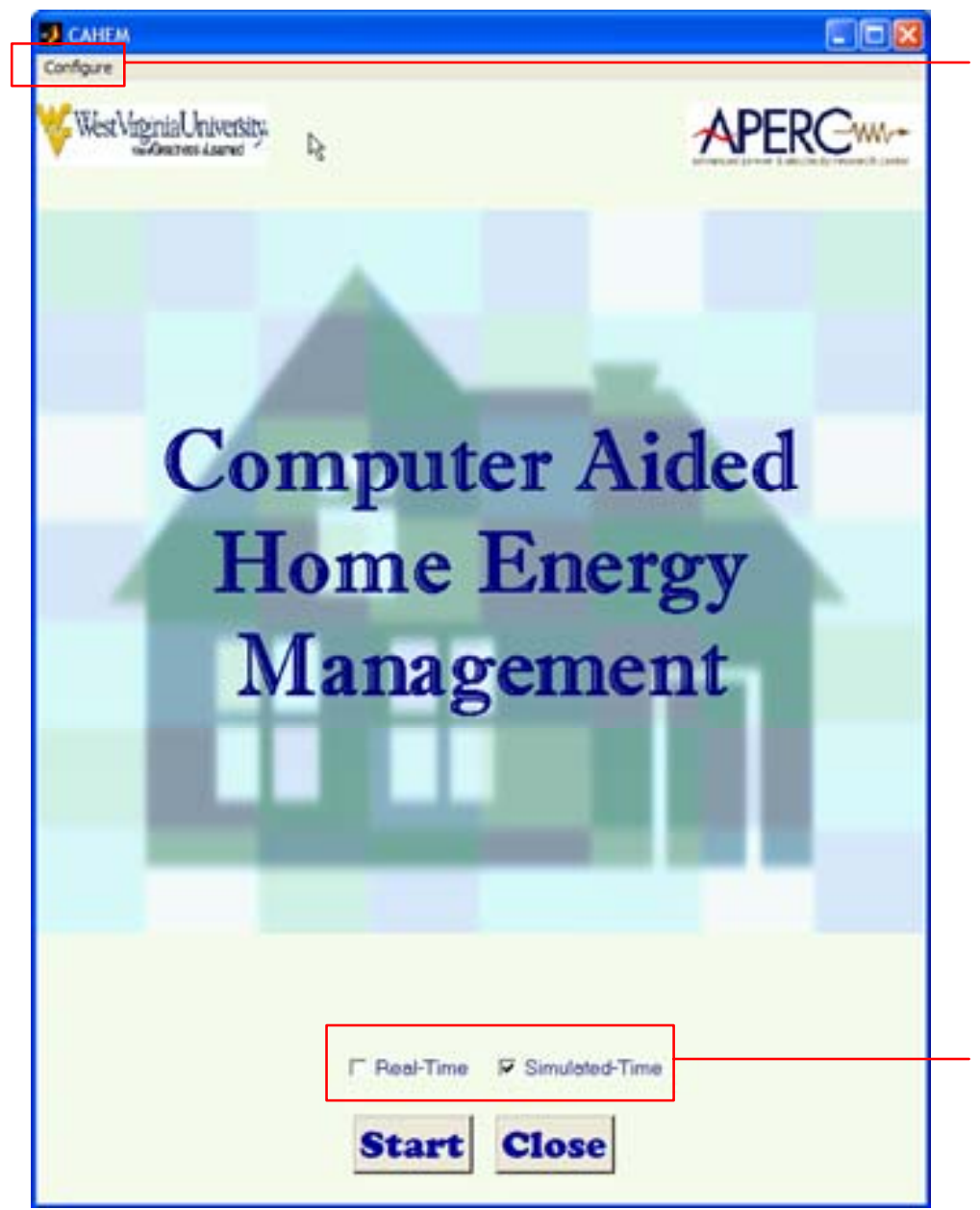

Figure 22: The CAHEM User Interface 


\section{Step 2: Configuration of Hardware and Load Models}

Clicking on Configure in the menu of the CAHEM window opens the Configuration window shown in Figure 23. Select an appliance and the appropriate House Code and

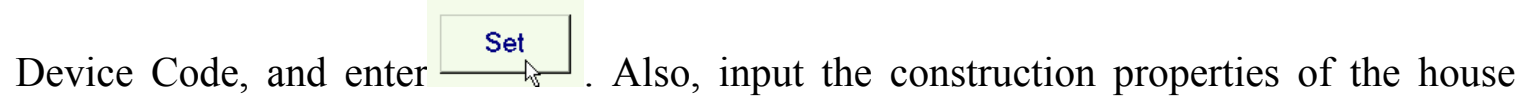
using the edit boxes and the list boxes.

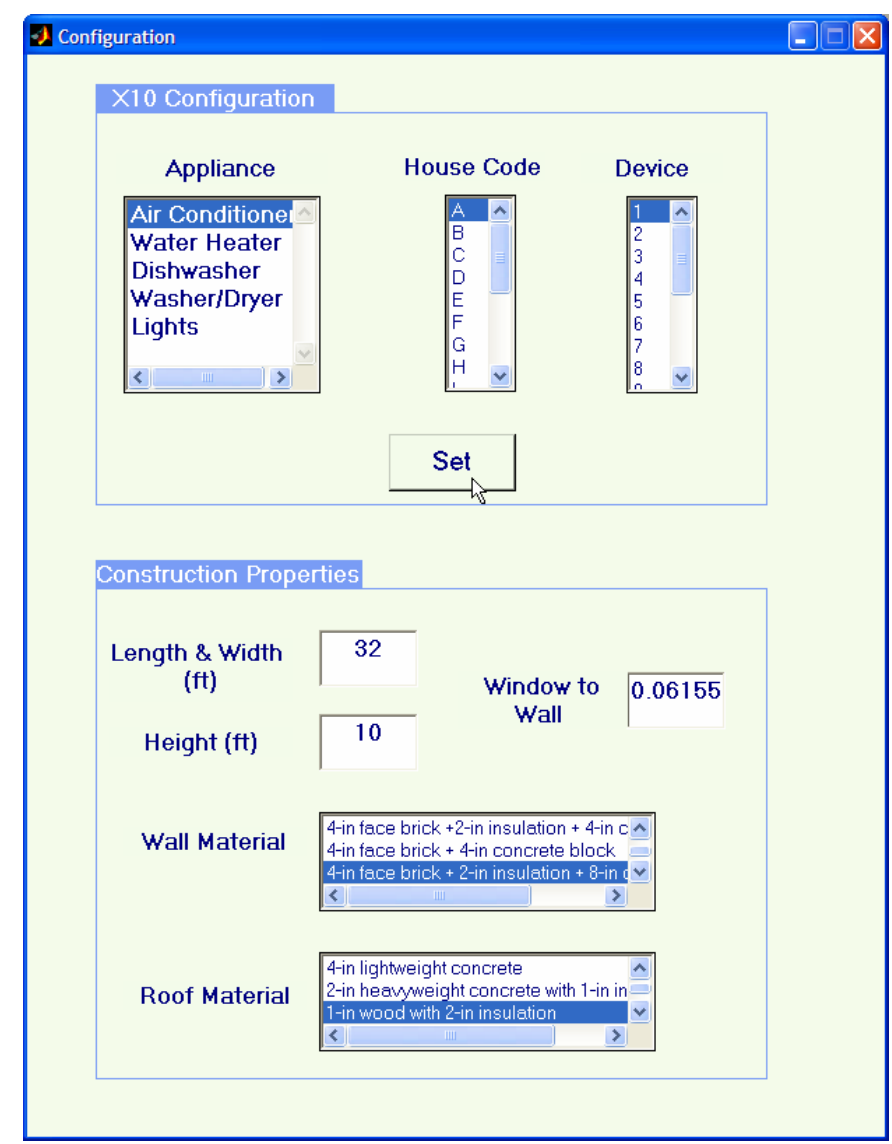

Figure 23: The CAHEM User Interface - Configuration

\section{Step 3: Load Control and Appliance Settings}

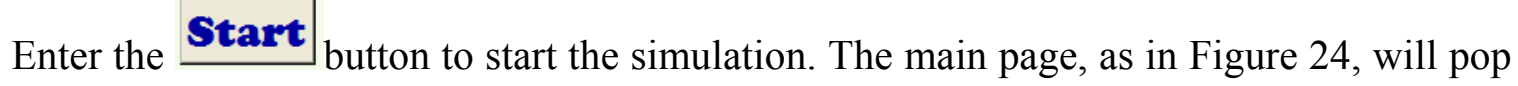
up. Select the mode of operation from the Modes pane. By default, it will operate in economy mode. The graph shows hourly electricity consumption, price, ambient temperature, as well as thermostat setting, for the time window of 5 hours ending at the current hour. Use the popup menu to select the quantity you wish to monitor. The graphs 
are updated each simulated hour. The red arrow indicates the present hour. The class of each hour is represented with the following convention. The definitions of hour classes, peak, off-peak, and pre-peak, are defined in the next subsection.
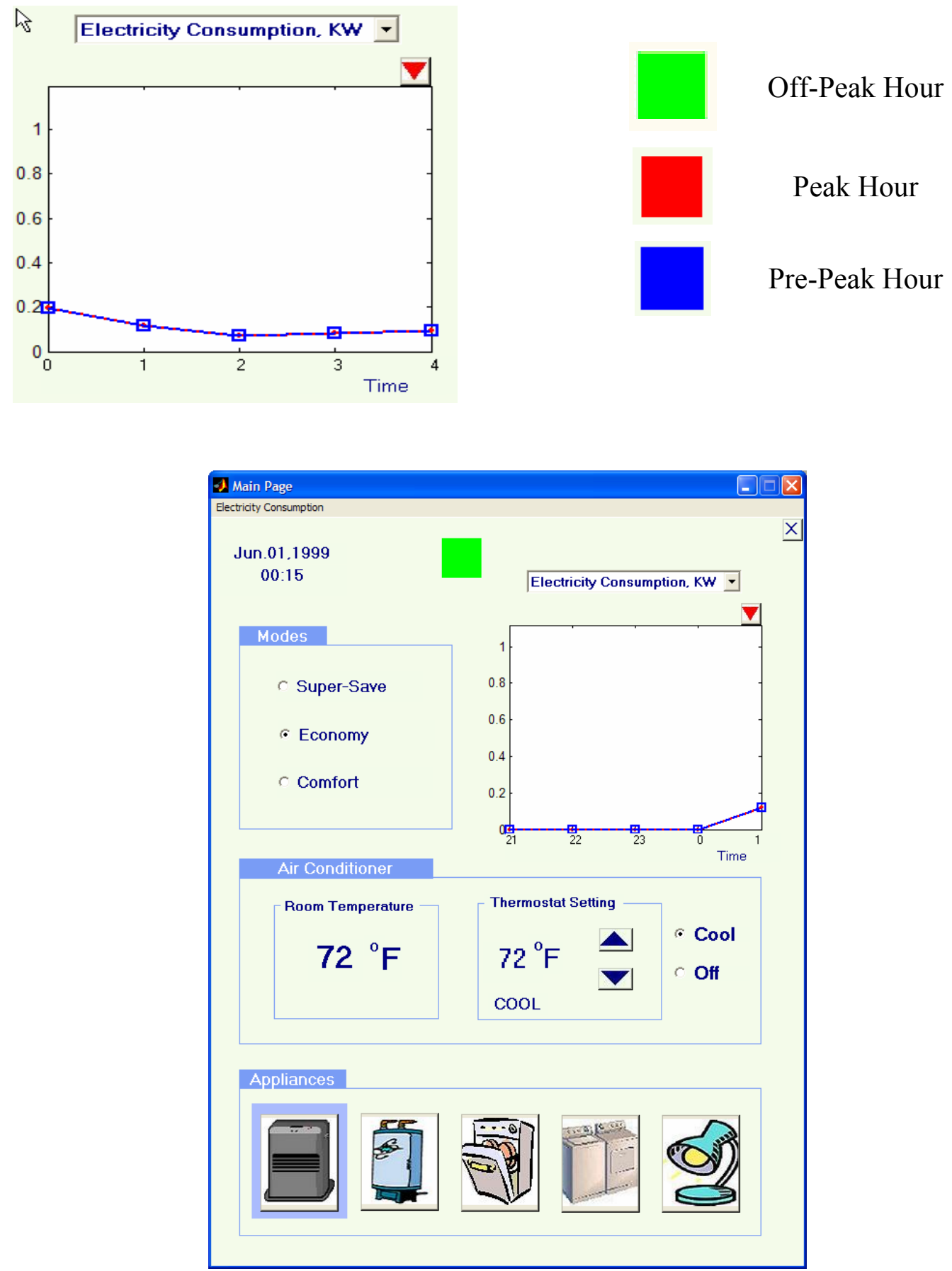

Figure 24: The CAHEM User Interface - Main Page 
Select the appliance to be controlled from the Appliances pane. The selected appliance will be highlighted. Table 3 summarizes how appliances can be controlled with the CAHEM user interface.
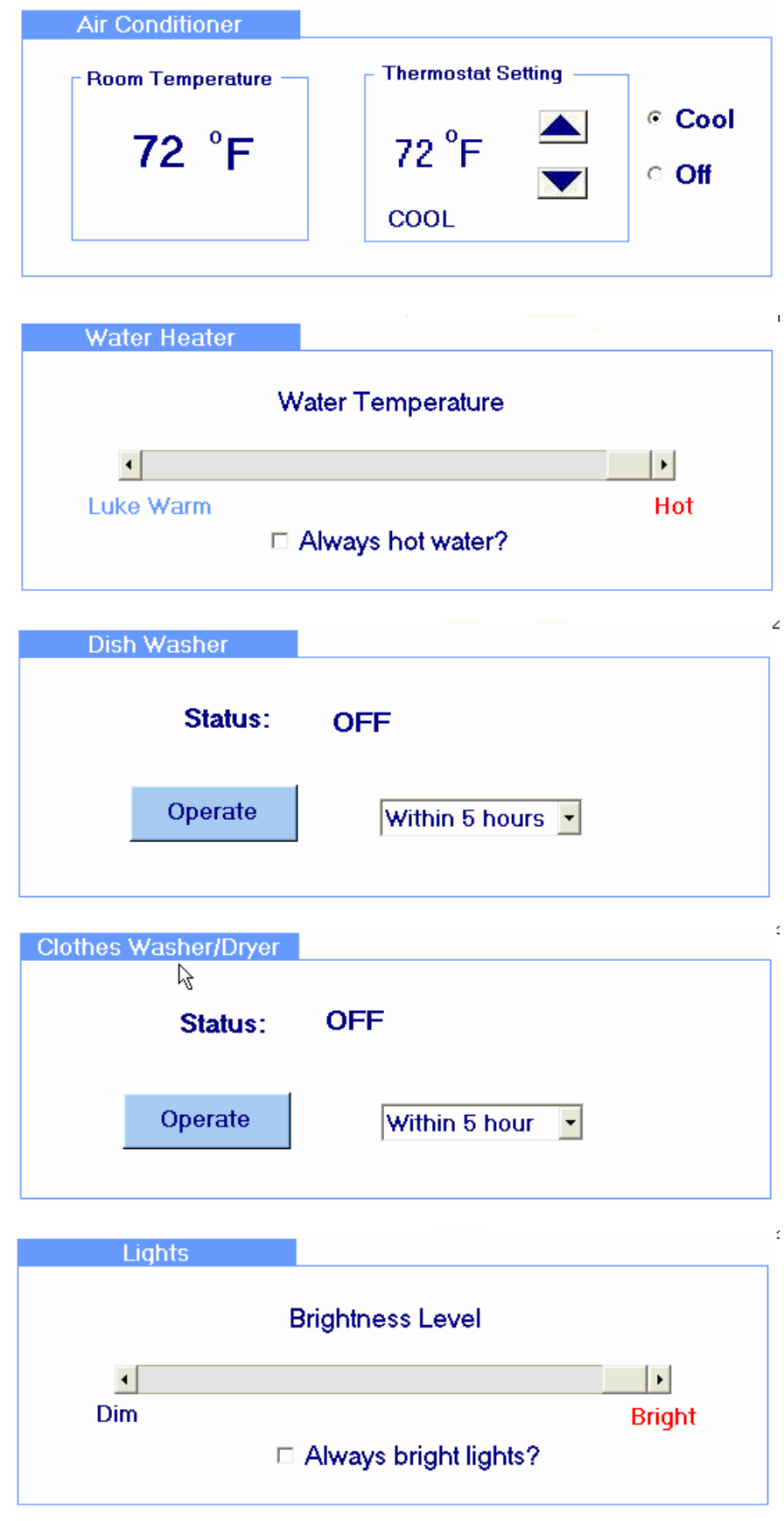

1. Displays room temperature

2. Sets and displays the thermostat setting

3. Air conditioner control - 'Cool' and 'Off'

1. Displays and sets the status of the water heater

2. Select 'Always hot water?' if no load control for the water heater is desired.

1. Displays the status of the dish washer- 'On', 'Off', or 'Scheduled'

2. Allows scheduling or operating of additional dishwashing loads.

1. Displays the status of the washer/dryer- 'On', 'Off', or 'Scheduled'

2. Allows scheduling or operating of additional loads.

1. Displays and sets the status of the lights

2. Select 'Always bright lights?' if no load control for lights is desired.

Table 3: Residential appliance control 


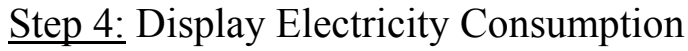

By clicking on the Electricity Consumption menu tab in the main page, the contribution of each appliance towards hourly electricity consumption for a time window of 5 hours can be monitored. Select the desired appliance from the popup menu shown in Figure 25.

\begin{tabular}{|l|}
\hline Air Conditioner \\
\hline Air Conditioner \\
\hline Water Heater \\
Dishwasher \\
Washer/Dryer \\
Lights \\
\hline
\end{tabular}

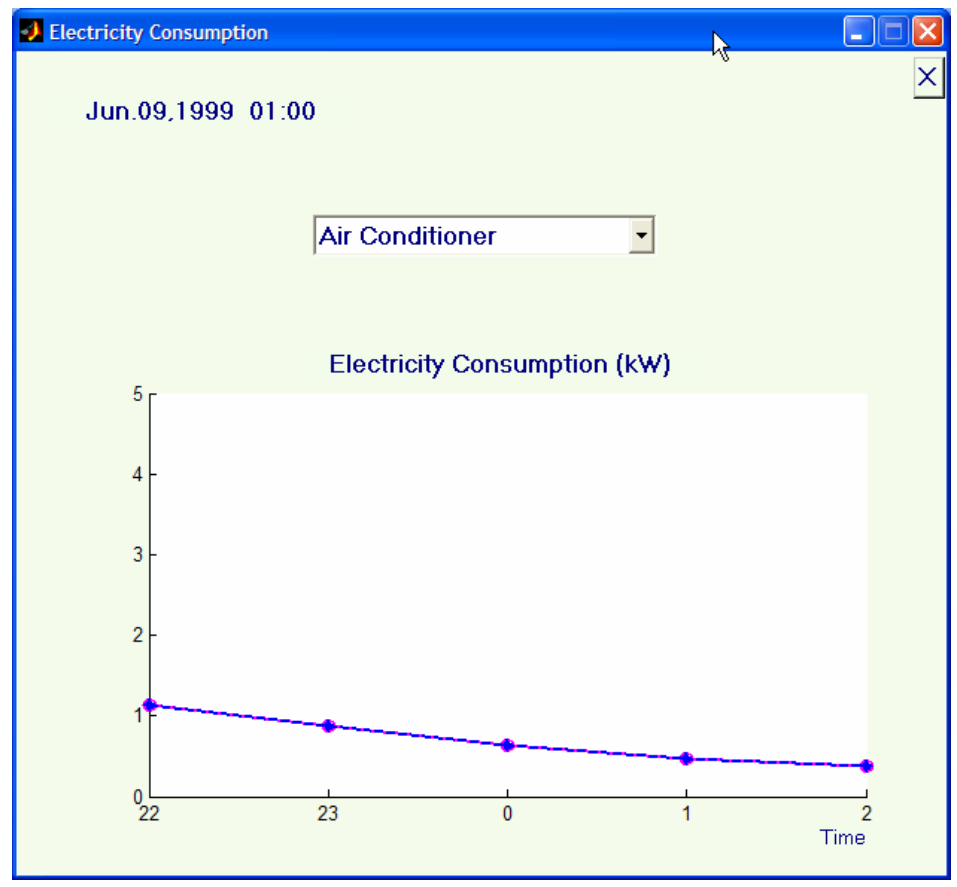

Figure 25: Appliance-wise electricity consumption profiles

Step 5: Stop Simulation

Click on the cross $\frac{X}{}$ in the Main Page to stop simulation. 


\subsection{Price Responsive Load Management Strategy}

The load management algorithm is the brain of the CAHEM system. A prime function of this algorithm is to adjust the operational settings of the appliances depending on price, load, and weather inputs from the LSE or utility. The consumer preferences govern the limits of appliance control. The consumer preferences essentially define comfort criteria, including thermal comfort of air, thermal comfort of hot water, and visual comfort as a result of illumination. The controlling parameters for the various appliances are specified below.

> Central Air Conditioner: Thermostat Setting and Temperature Tolerance

D Water Heater: Relative Water Hotness

$>$ Lights:

Relative Brightness Level

$>$ Washer/Dryer:

Tolerable Delay in Rescheduling

$>$ Dishwasher:

Tolerable Delay in Rescheduling

Assume for a moment that the system is under equilibrium; thus, supply equals demand exactly. Though the electricity price follows a supply curve, a supply curve itself varies on a daily or hourly basis. Figure 26 represents uncertainty in supply and the price relation obtained for the 1999 PJM summer market period with generating capacity assumed constant at $55 \mathrm{GW}$. The spread of points in Figure 26 indicates that for a given price, supply can vary widely and vice-versa.

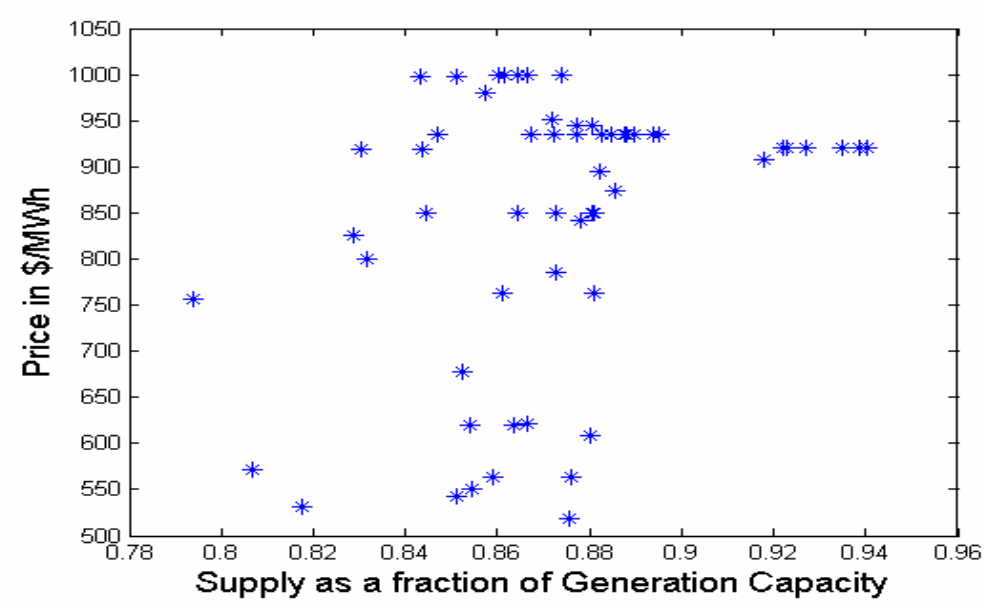

Figure 26: Uncertainty in supply and price relation 
The price-load relationship is a very complex nonlinear process. If the severity of the condition of a power system is to be judged based on price and load data, the decision boundary is not well-defined.

Additionally, customer preferences for comfort are important in this algorithm. Definition of comfort includes thermal comfort of air, thermal comfort of hot water, and visual comfort as a result of illumination. The quantitative description of comfort is not possible for the customers. However, they can describe comfort requirements in vague linguistic terms.

These factors motivate the use of fuzzy logic for the load controller. Fuzzy logic allows the control variables to be defined to have unclear boundaries. The controller can incorporate a simple rule-based inference system in which intuitively defined fuzzy rules govern the output of the controller [36].

\section{Classification of Hours}

1. PEAK hours:

As the name suggests, PEAK hours are the hours in which the power system is at a critical load. The incidence of high load is reflected by a price spike. The controller must schedule appliance operation so as to alleviate constraint on the system by reducing or shifting some loads from peak hours. In general, the controller is of a more preventive nature if more hours are marked as peak hours by the controller.

\section{OFF-PEAK hours:}

OFF-PEAK hours are the normal operating hours in which the demand is supplied smoothly. Off-peak hours are characterized by non-critical load and price. In load shifting strategies, these hours are used to compensate peak hour load reduction.

\section{PRE-PEAK hours:}

In the case of N-hour-ahead or day-ahead notification, the off-peak hours before peak hours can be utilized to minimize discomfort to the customers during peak hours. Such 
hours can be classified as PRE-PEAK hours. For example, with an air conditioner set to a lower temperature, the thermal inertia of a house will reduce the discomfort during peak hours when demand is reduced by setting the air conditioner to a higher temperature.

The classification of hours is carried out by a fuzzy inference system. A global template defining fuzzy sets is shown in Figure 27. The parameters of the membership function are tuned according to the customer selection. This fuzzy system determines the confidence that the hour under consideration is the peak hour or is not the peak hour, based upon the price and load data. For generality of design, normalized data was used.

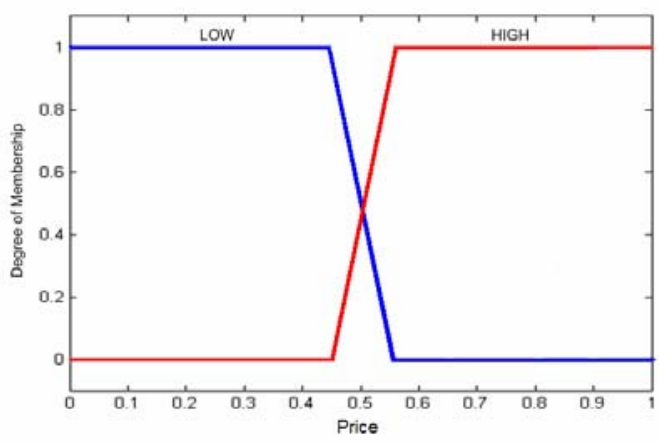

a) Price

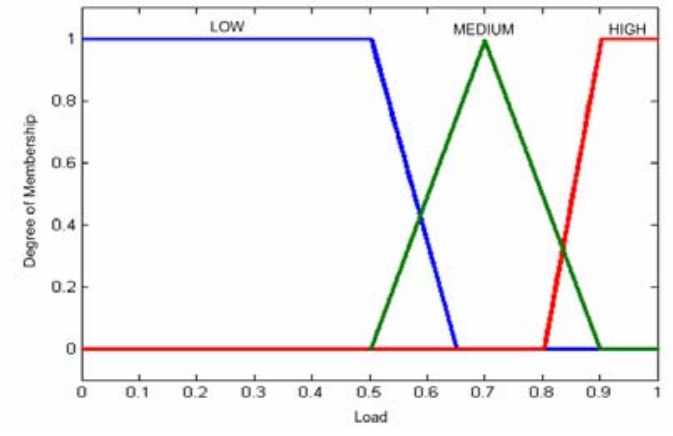

b) Load

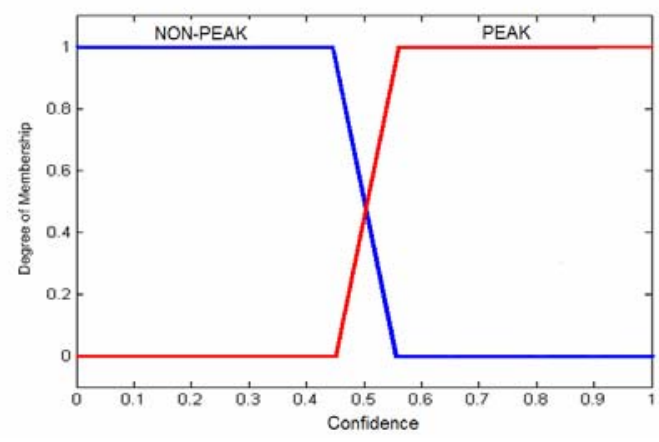

c) Confidence

Figure 27: Membership functions for the Hour Classification fuzzy unit

The fuzzy rule-based system uses the following rules:

- PEAK hour rules

1. If Load is HIGH and Price is HIGH, then the hour is PEAK hour.

2. If Load is MEDIUM and Price is HIGH, then the hour is PEAK hour.

3. If Load is HIGH and Price is LOW, then the hour is PEAK hour. 
4. If Load is LOW and Price is HIGH, then the hour is PEAK hour.

- NON-PEAK hour rules

1. If Load is not HIGH and Price is LOW, then the hour is NOT a PEAK hour.

Figure 28 shows the peak hour detection results for the 1999 PJM summer data with the fuzzy membership functions shown in Figure 27.

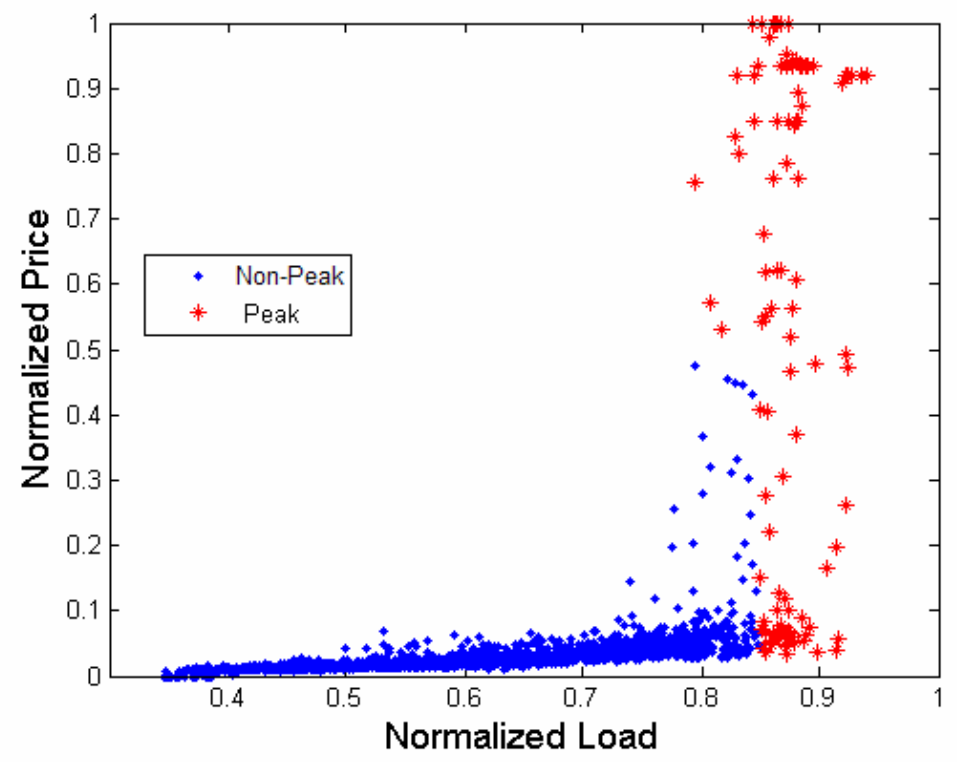

Figure 28: Detection of peak hours (1999 PJM)

The extent of advanced price notification plays a very important role in the development of the load control algorithm. The load shifting to hours before peak hours is possible if the load controller receives advance notification. The requirement of pre-peak hours is dependent upon severity and duration of the peak period. The number of pre-peak hours equals the number of peak hours.

Before explaining the details of the load control algorithm, let us discuss various scenarios depending on how far in advance the price signals are available. 


\section{Case 1: Hour- Ahead Notification}

In this scenario, prices are assumed to change each hour. Hourly price and load signals are available just a few minutes before the start of the corresponding hour. This scheme provides minimum advance notification, which also implies that there is very limited future information available for the load control algorithm. If the algorithm finds an incidence of peak period, it will reduce the electricity demand by modifying operational settings. For example, the thermostat setpoint will be set to a higher temperature and/or lights will be dimmed. The compensation for the load reduced during the peak period is not possible as a result of limited advance information.

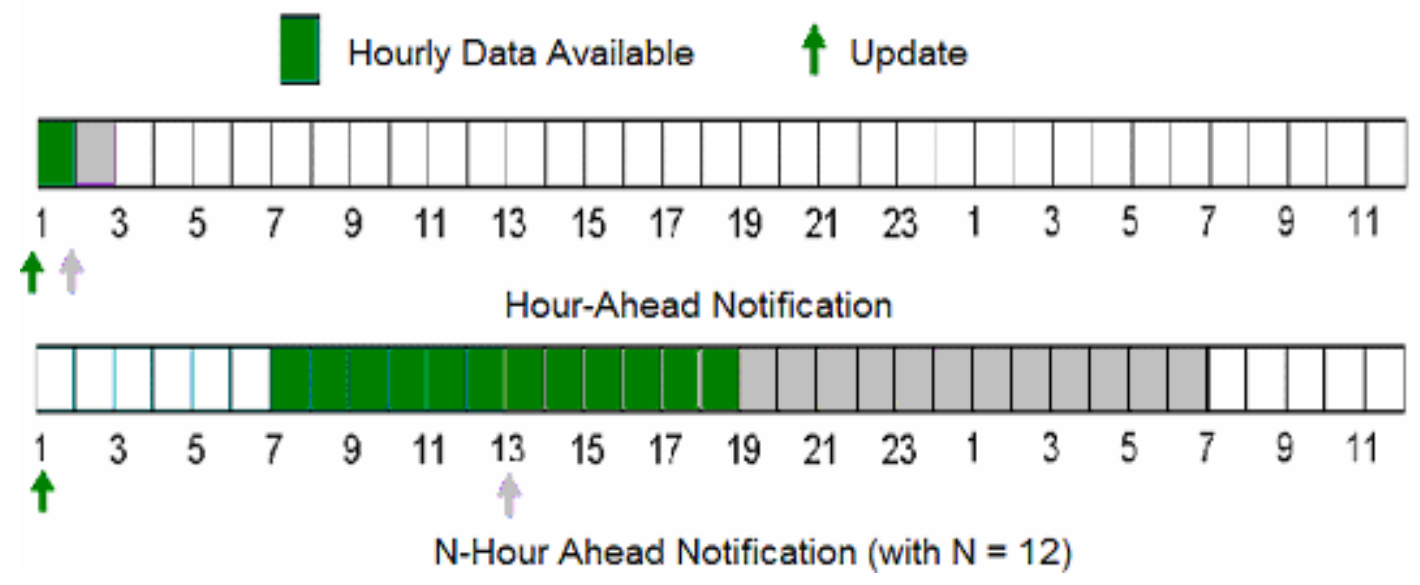

Figure 29: Notification scenarios

The load control algorithm with an hour-ahead notification, sketched in Figure 30, is essentially similar to the load shedding algorithm. Figure 31 represents the expected effect of load control with an hour-ahead notification on the residential load profile. 


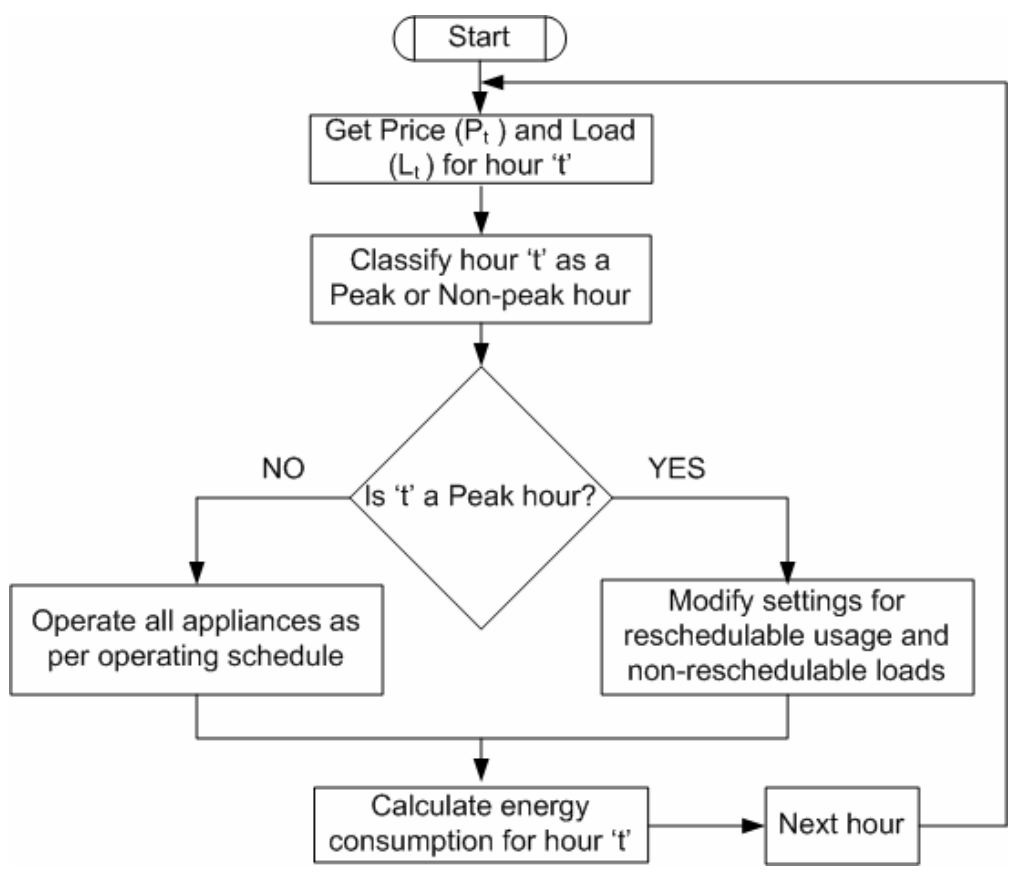

Figure 30: Flowchart for load control with hour-ahead notification

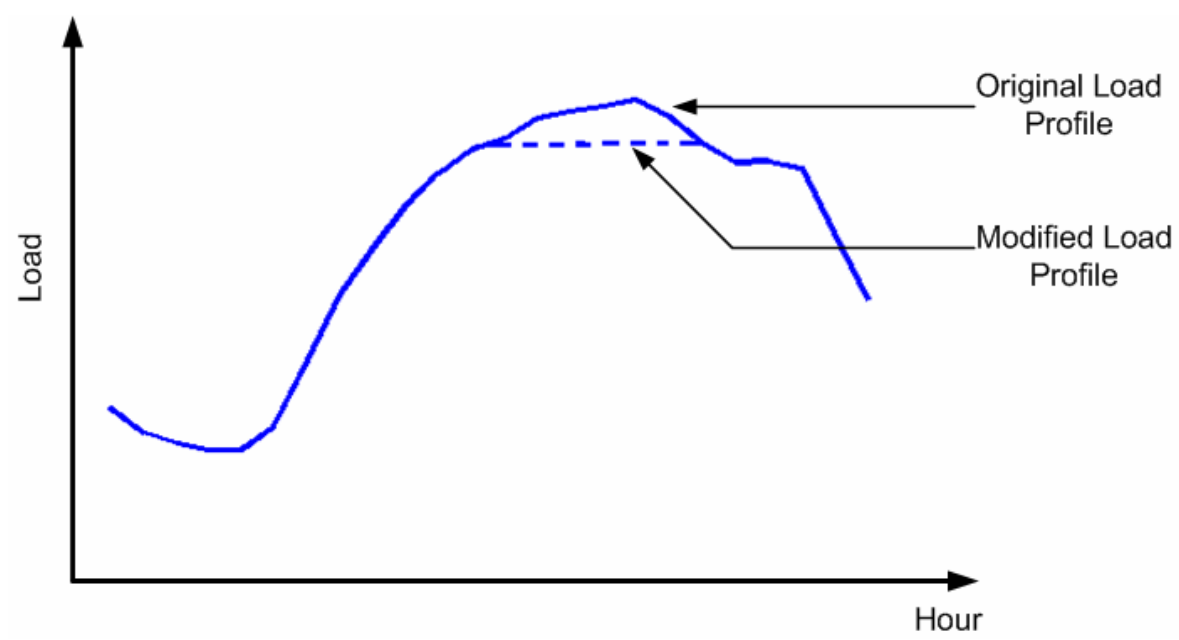

Figure 31: Load profile with control based on hour-ahead notification

\section{Case 2: N-Hours-Ahead Notification}

With N-hours-ahead notification, the prices for a time frame of $\mathrm{N}$ hours is available in advance, say $\mathrm{N} / 2$ hours. If $\mathrm{N}$ is sufficiently large, future information can be utilized in deciding appliance operation schedules in advance. It is clear from Figure 29 that an an hour the information of the next $\mathrm{N} / 2$ hours is available. With future information, the 
CAHEM can schedule appliances well in advance. The reschedulable usage can be rescheduled so as to minimize the discomfort. The overall load control strategy with an N-hour-ahead notification is summarized in Figure 32.

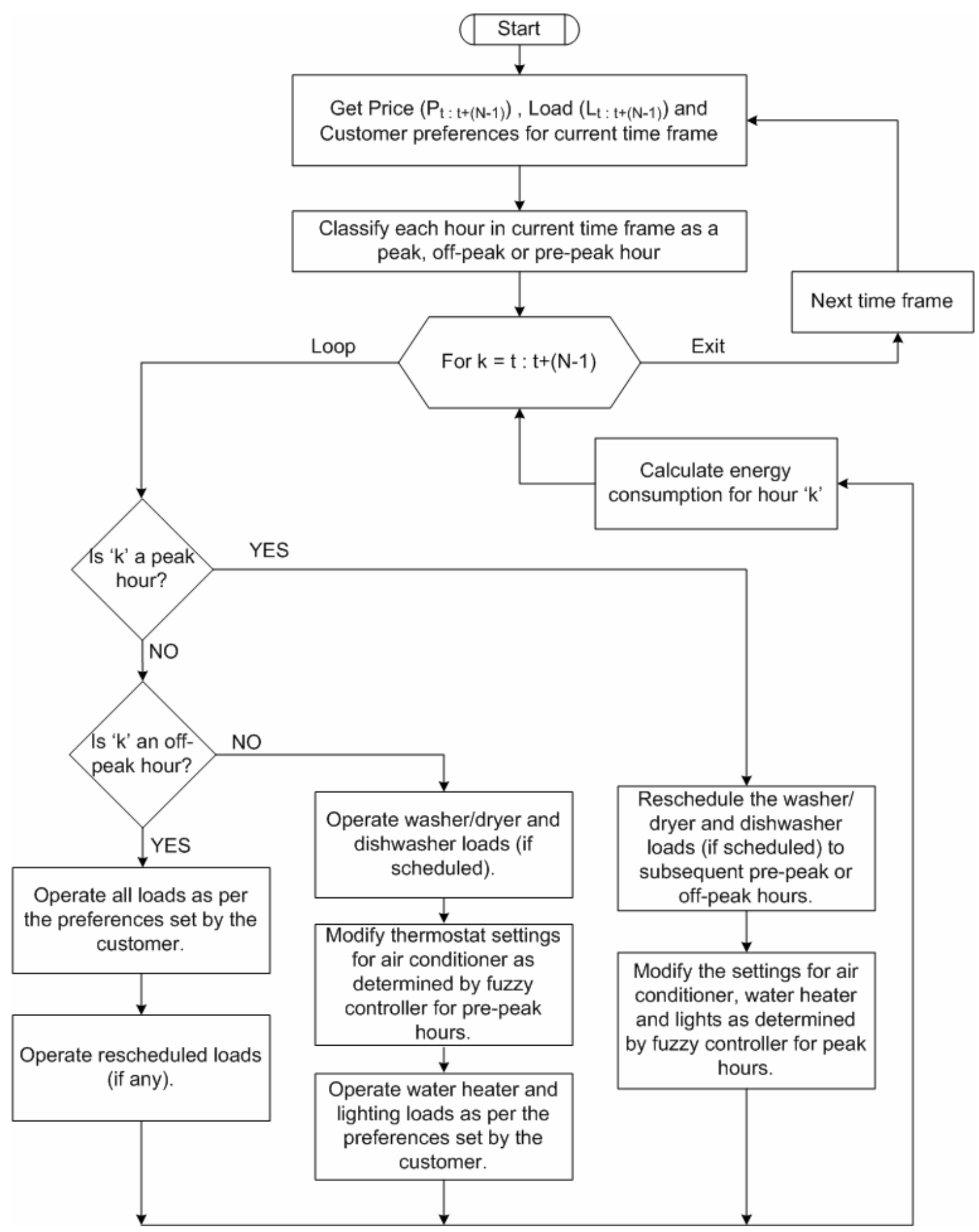

Figure 32: Flowchart for the appliance control under CAHEM 
As shown in Figure 33, N-hours-ahead notification allows the load to be shifted from a few critical hours of the day to the remaining hours.

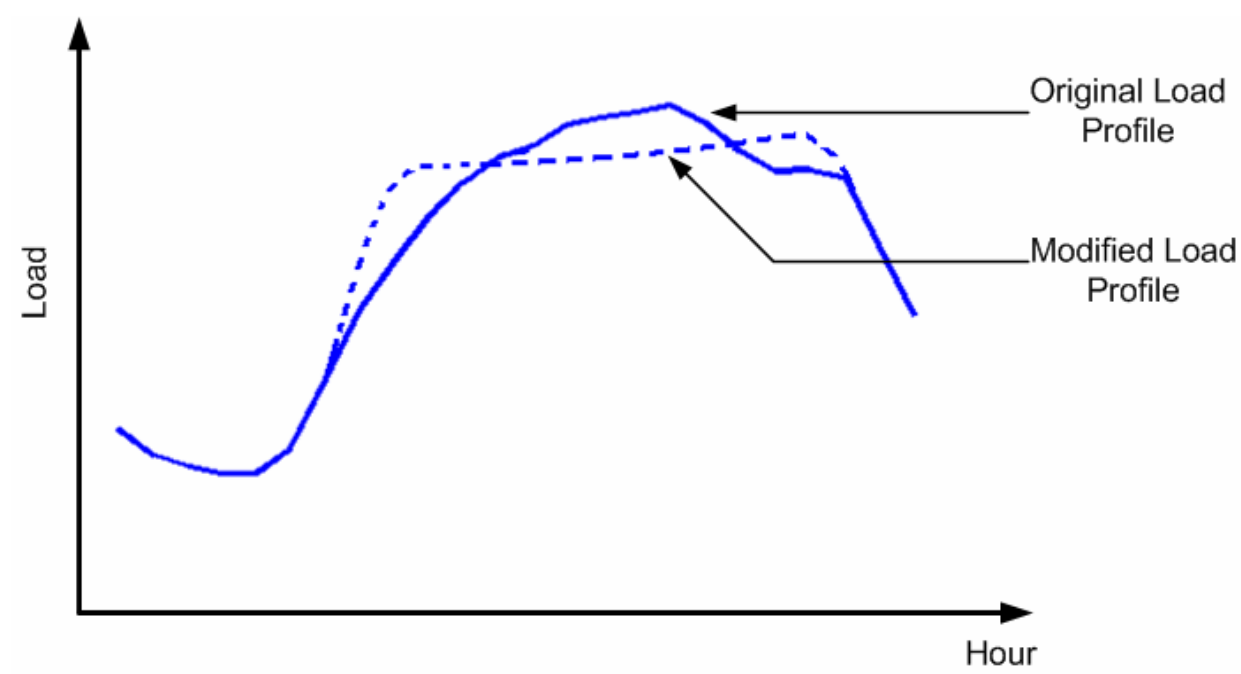

Figure 33: Load profile with control based on N-hour-ahead notification

\section{Load control during the peak hours}

During peak hours, the CAHEM tries to minimize electricity usage for residential appliances while maintaining the customer's comfort within acceptable margins. If the washer/dryer or dishwasher is scheduled during the peak hour, then reschedule its operation to any pre-peak or off-peak hour within the maximum allowable hours specified by the customer. The controller reschedules the reschedulable services to any of the off-peak hours if it is within the maximum delay set by customer. In the absence of the off-peak hour in the delay span for operating reschedulable services, the services are scheduled during the peak hour with the lowest price. If the reschedulable services are rescheduled at the lowest price hour, then it will create a new peak load hour. Therefore, the rescheduling is done randomly within the acceptable hours. The operation of an air conditioner, water heater, and lights is decided by the fuzzy controller based upon the price and the load. The thermostat setting for the air conditioner is set to a higher temperature so as to reduce the cooling load. The fuzzy controller also takes into account if non-reschedulable loads are operated during the peak hour under consideration. The fuzzy controller for peak hours is described below. 
1) Load: It is assumed that the power system is capable of supplying the load at the worst time equal to the generating capacity, though with the high risk of outages. As peak load is a system dependent parameter, a normalized load, which is a fraction of generation capacity, is used. The load is described as low, medium, or high. Medium and high loads are very likely during the peak hours. The range for the membership function may vary with changes in customer preferences. The global template is shown in Figure 34-a.

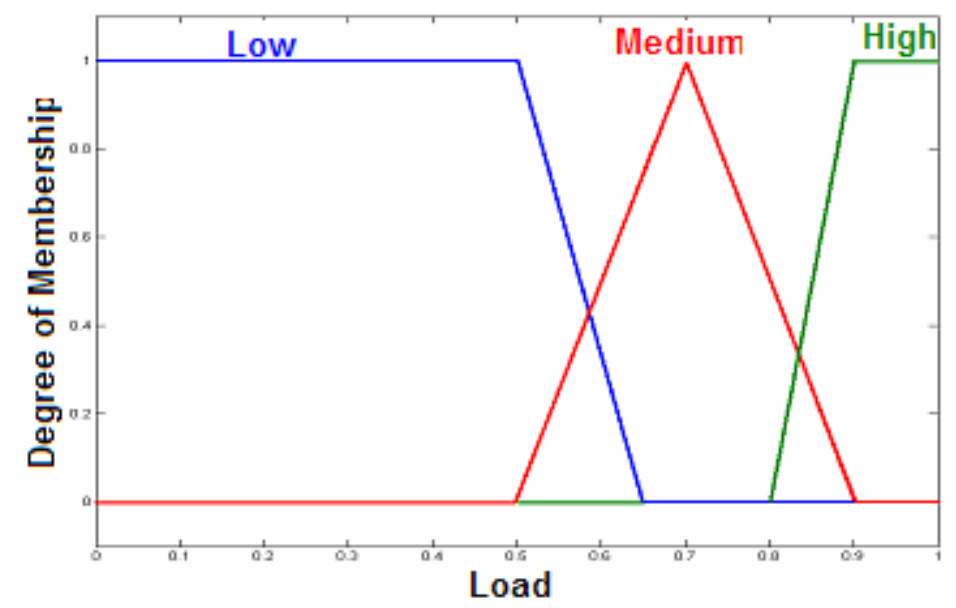

Figure 34 - a: Membership function for Load

2) Price: Similar to load, price is also a system dependent. Three fuzzy sets are defined for price: Low, Medium, and High. Again, the price is normalized for the sake of generality. The global template for price is shown in Figure 34-b.

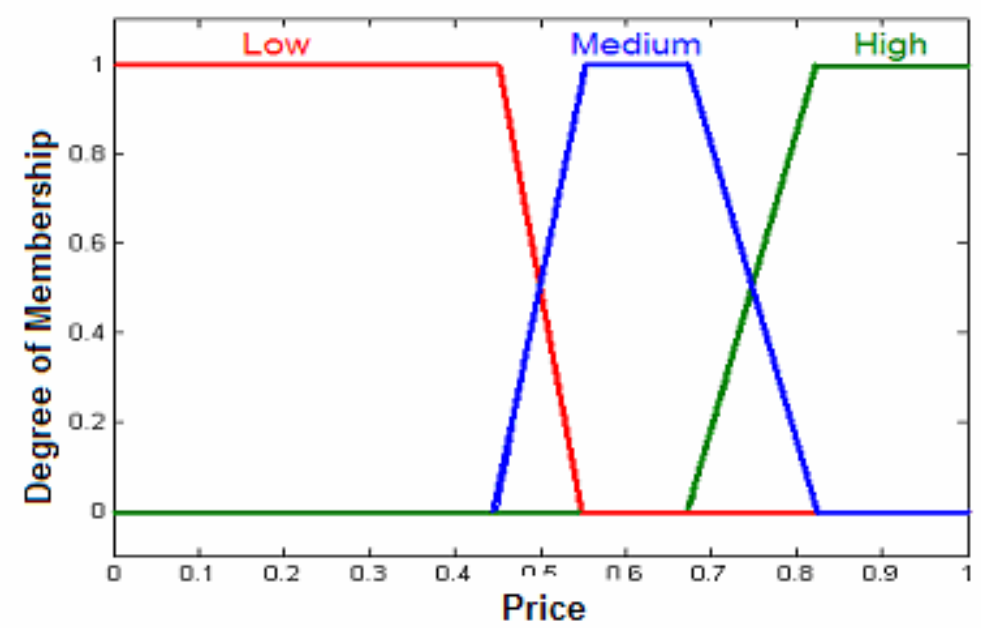

Figure 34 - b: Membership function for Price 
3) Thermostat Setting: The thermostat setting is determined based upon price and load data. The temperature is classified into the following three sets- Desired, Warm, and Warmer. The spread of the membership function will change according to customer preferences.

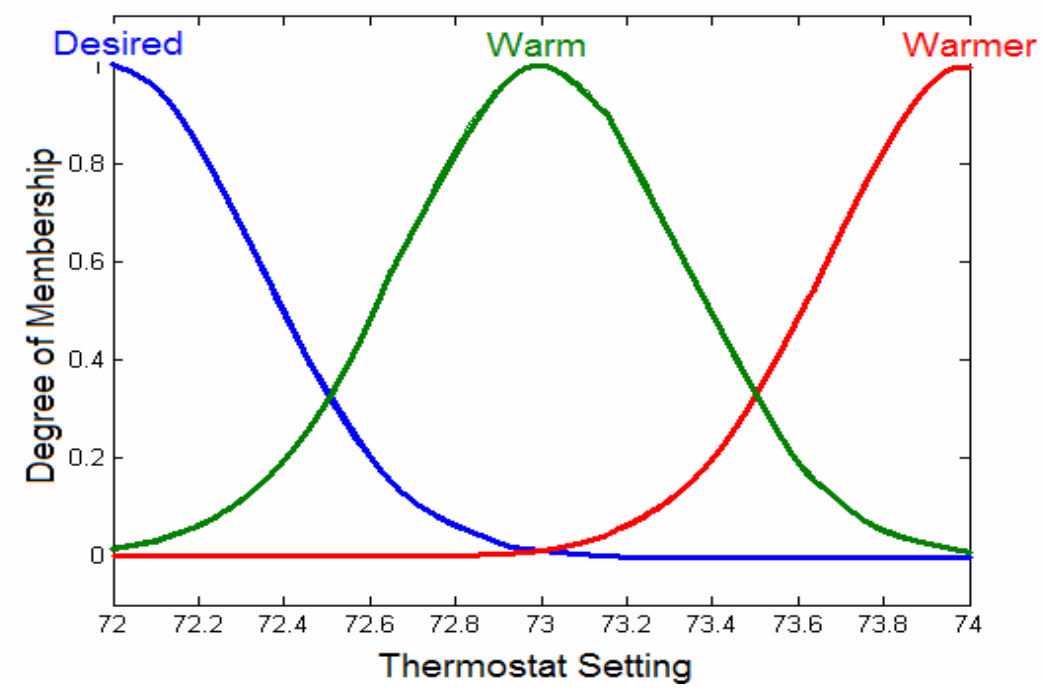

Figure 34 - c: Membership function for Thermostat Setting

4) Water Heater: The fraction of the desired water heater performance is determined by a fuzzy model. The performance of the water heater is reflected in the water temperature. The water heater performance is defined as: desired, warm, or cool.

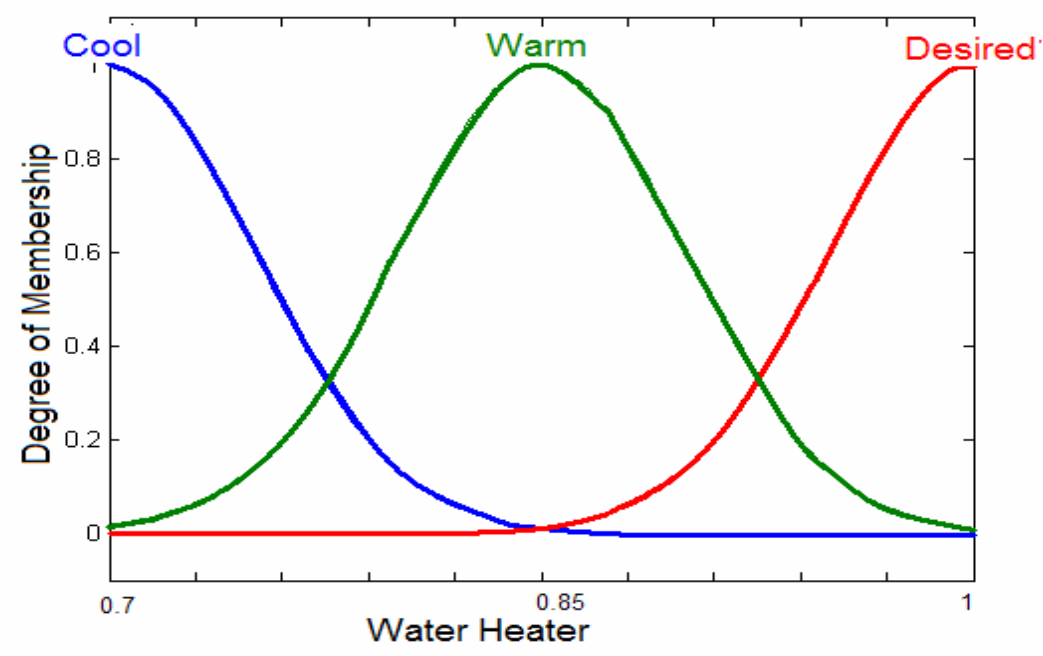

Figure 34 - d: Membership function for Water Heater 
5) Lights: The brightness of lights is defined by desired, dim, and dimmer. The global template is shown in Figure 34-e.

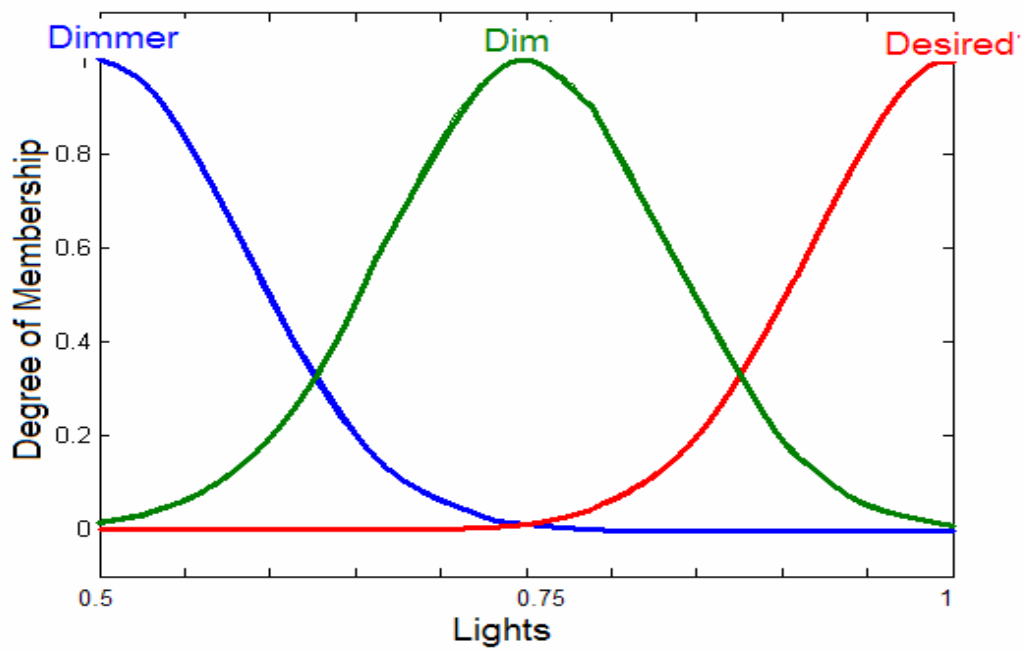

Figure 34 - e: Membership function for Lights

Fuzzy Rules:

1. If the load is not high and the price is low, then the thermostat setting, water heater, and lights are at the desired settings.

2. If the load is high and the price is low, then the thermostat setting is warm, water heater is warm, and lights are dim.

3. If the load is high and the price is medium, then the thermostat setting is warm, water heater is cool, and lights are dimmer.

4. If the load is not high and the price is medium, then the thermostat setting is warm, water heater is warm, and lights are dim.

5. If the load is high and the price is high, then the thermostat setting is warmer, water heater is cool, and lights are dimmer.

6. If the load is medium and the price is high, then the thermostat setting is warmer, water heater is warm, and lights are dim.

7. If the load is low and the price is high, then the thermostat setting is warm, water heater is warm, and lights are dim. 
In Figures 35-a through 35-c, the surface plot for the fuzzy controller is shown. It is a plot of how the controller's outputs - thermostat settings, water heater, and lights change as a function of the normalized load and the normalized price.

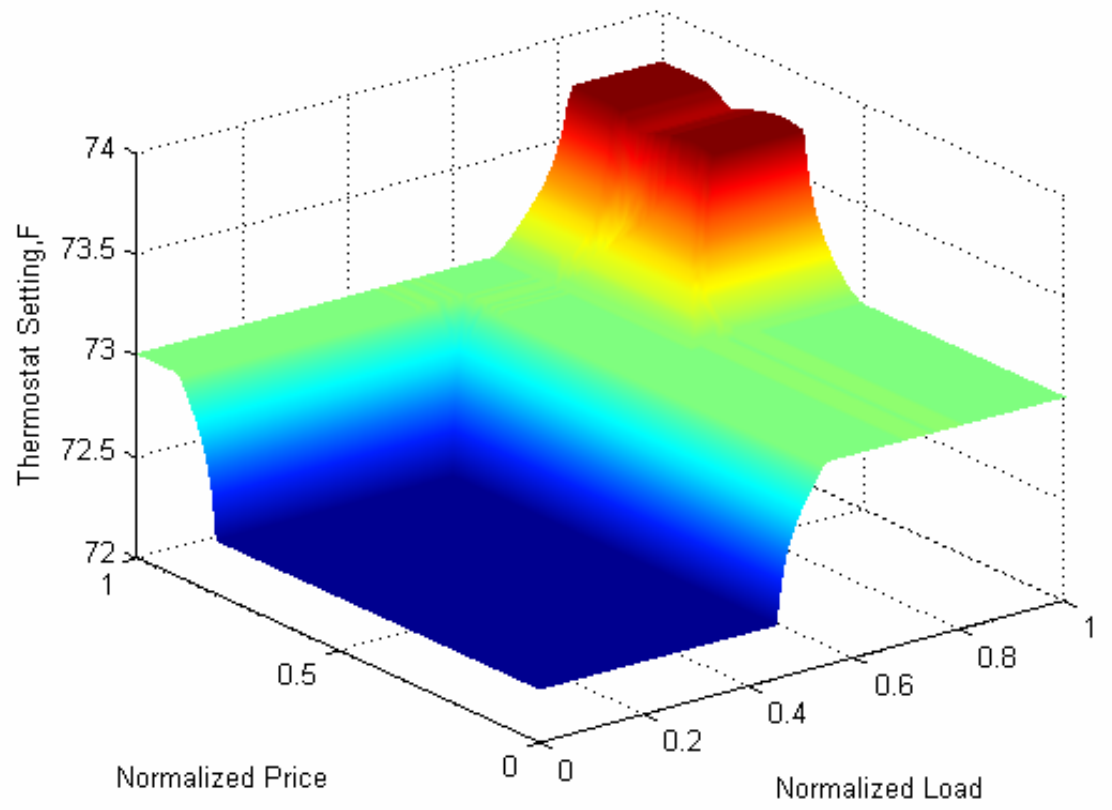

Figure 35-a: Surface plot for thermostat setting

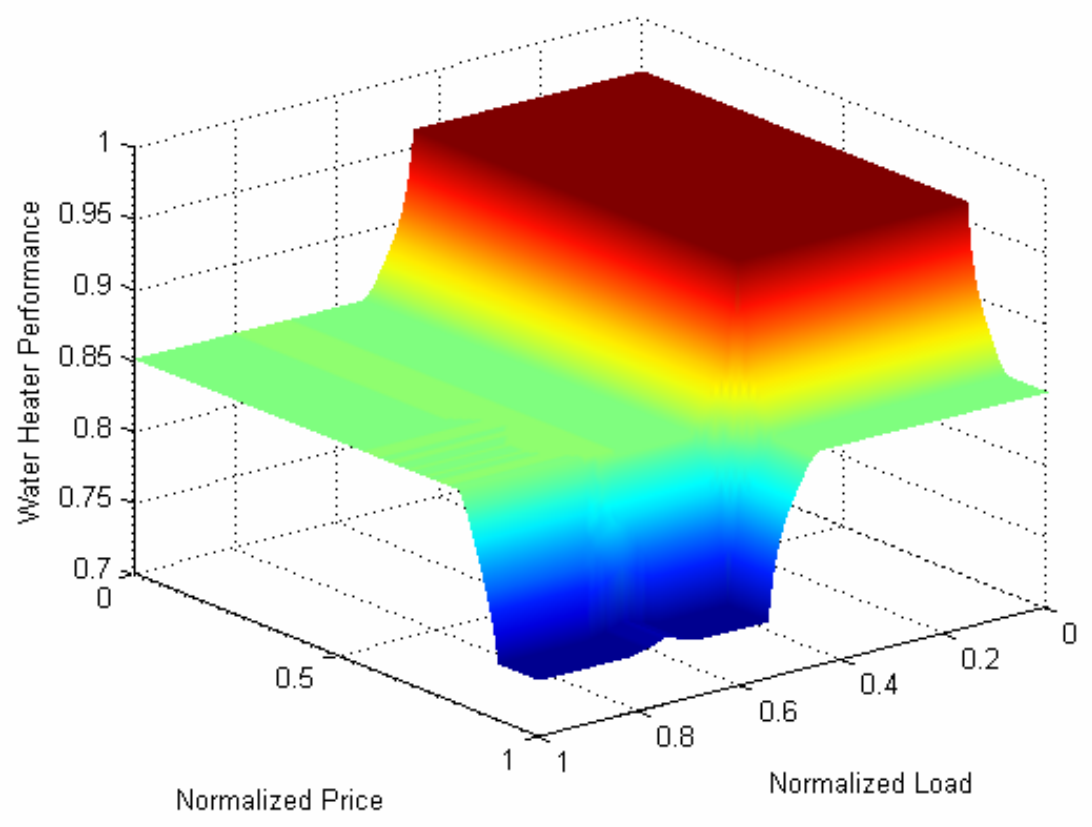

Figure 35-b: Surface plot for water heater performance 


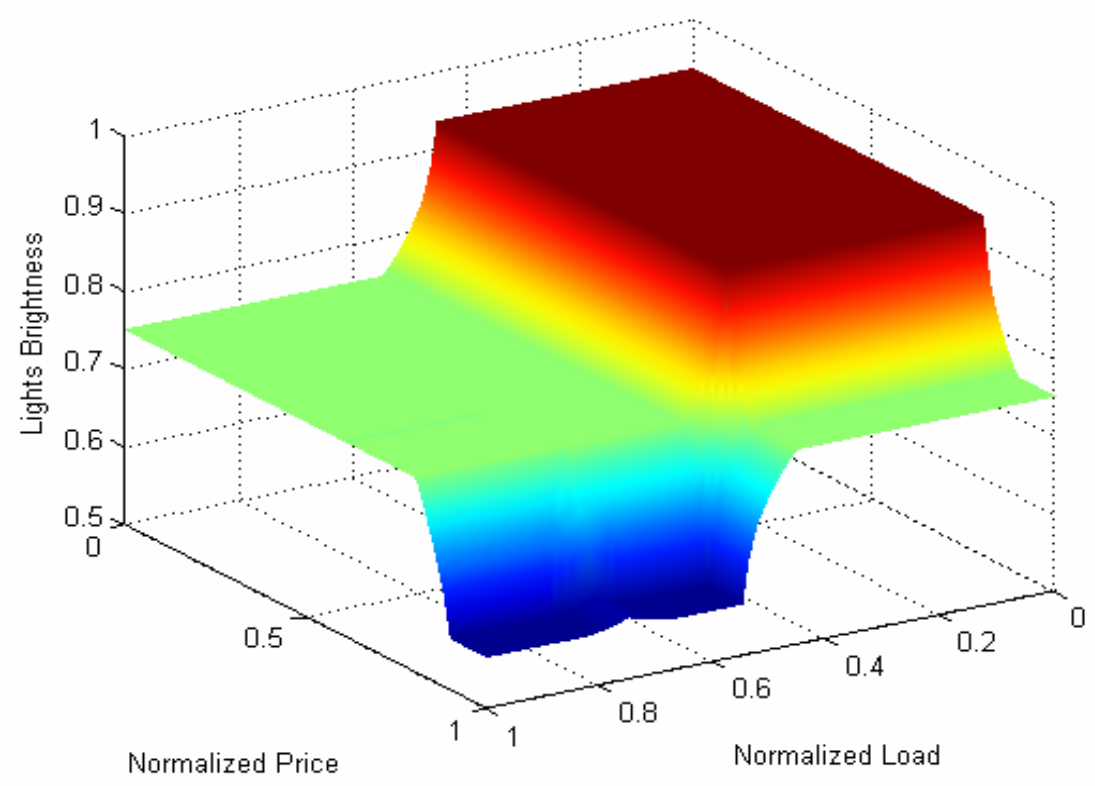

Figure 35-c: Surface plot for lights brightness

\section{Load control during the pre-peak hours}

The pre-peak hours are necessary for compensating the load reduction achieved during the peak hours. The N-hour-ahead notification enables the definition of the pre-peak hours. The pre-peak hours make use of the thermal inertia of a house by setting the thermostat to a lower temperature. This helps in reducing discomfort experienced during the peak hours. The pre-peak thermostat setting is determined by a fuzzy controller based on the price and load data. The originally scheduled or newly scheduled reschedulable loads are operated per the customer's preferences.

1) Load: The normalized load is defined as low, medium, or high. The range for the membership function may vary with changes in customer preferences. The global template for load is shown in Figure 36-a.

2) Price: Normalized price is described as low, medium, or high. The global template is shown in Figure 36-b. 
3) Thermostat Setting: The thermostat setting for the pre-peak hours is determined based upon load and price data. The thermostatic setting is defined as desired, cool, or cooler. The membership function parameters vary with customer preferences.

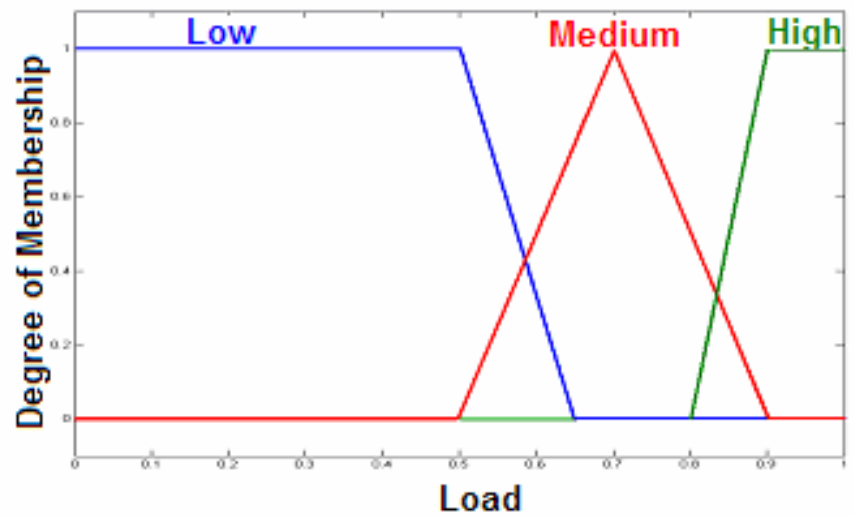

Figure 36 - a: Membership function for Load

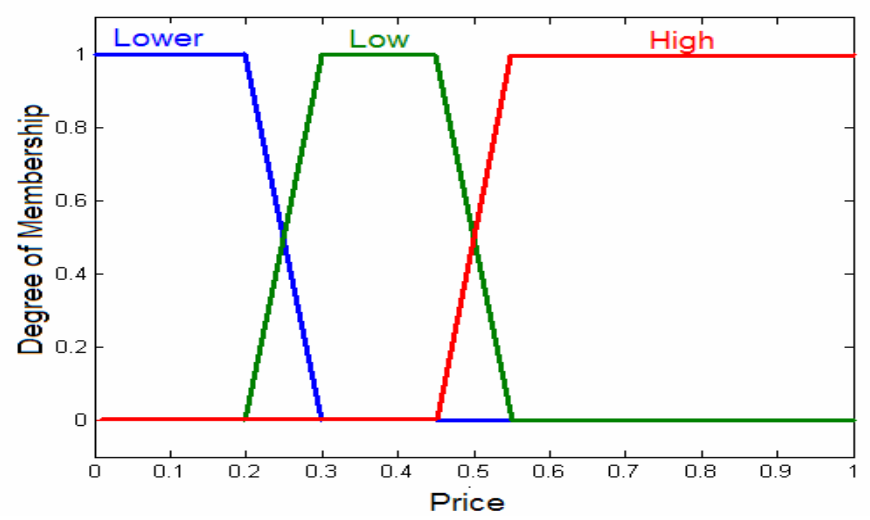

Figure 36 - b: Membership function for Price

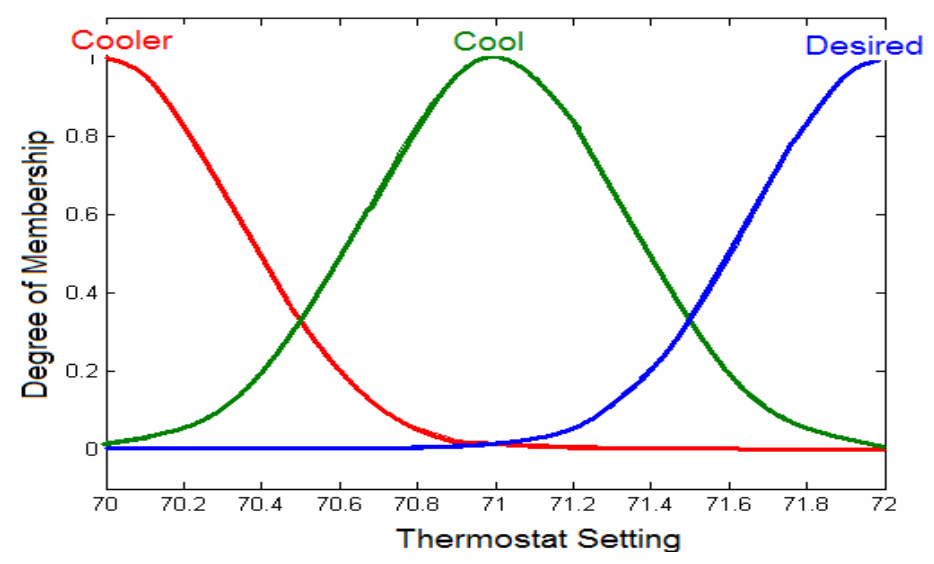

Figure 36 - c: Membership function for thermostat setting 


\section{Fuzzy Rules:}

1) If the load is high, then the thermostat setting is at desired.

2) If the load is medium and price is high, then the thermostat setting is at desired.

3) If the load is low and price is high, then the thermostat setting is at desired.

4) If the load is medium and price is not high, then the thermostat setting is cool.

5) If the load is low and price is low, then the thermostat setting is cool.

6) If the load is low and price is lower, then the thermostat setting is cooler.

The surface plot for the thermostat setting during the pre-peak hours is displayed in Figure 37.

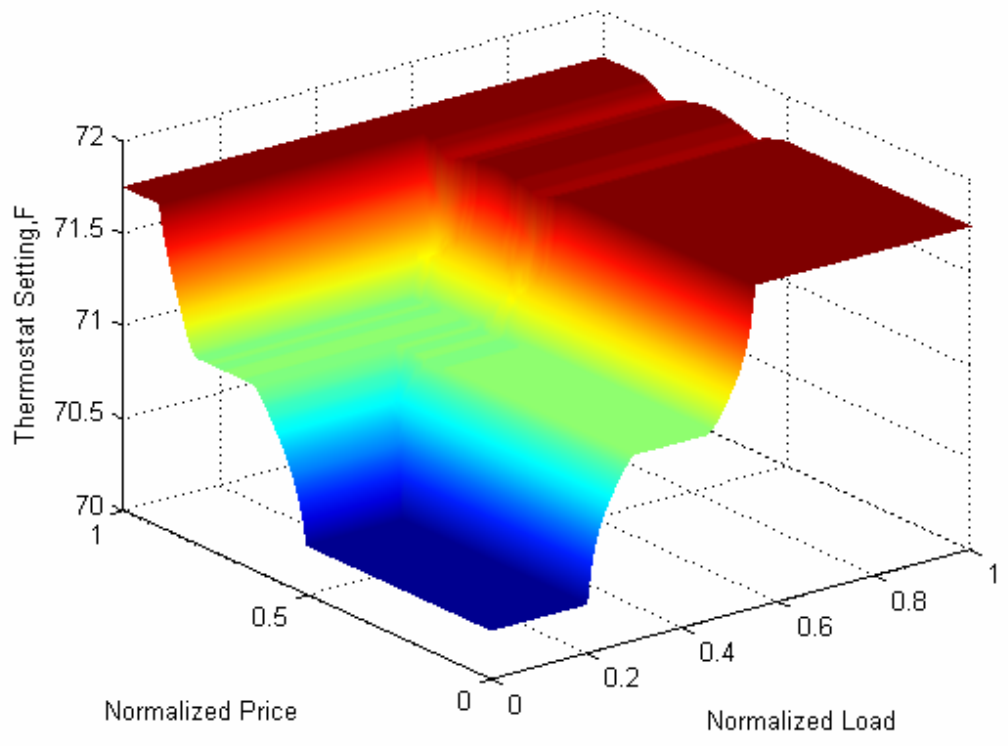

Figure 37: Surface plot for thermostat setting during pre-peak hour 


\section{Chapter 4}

\section{Results}

As the whole motive of the development of the CAHEM is to alleviate part of the peak load, the results can be appreciated by displaying their impacts on the peak system load. The CAHEM system places load control in the hands of the customer by giving the customer choices for various modes and preferences. Before discussing the results, the data used in the development, as well as testing the algorithm, will be explained.

\subsection{Simulation Data}

The evaluation of the load management system developed in this thesis is conducted with the hourly data from the Pennsylvania- New Jersey- Maryland (PJM) market for the duration of June 1, 1999 to August 31, 1999.

The load duration curve for the simulation period in Figure 38 indicates the summer peak load is $51.7 \mathrm{GW}$. Similarly, the price duration curve, shown in Figure 39, gives an idea about the occurrences of the high price periods. The price is limited to $999 \$ / \mathrm{MWh}$. The relation between the load and the price is given by the supply curve in Figure 40.

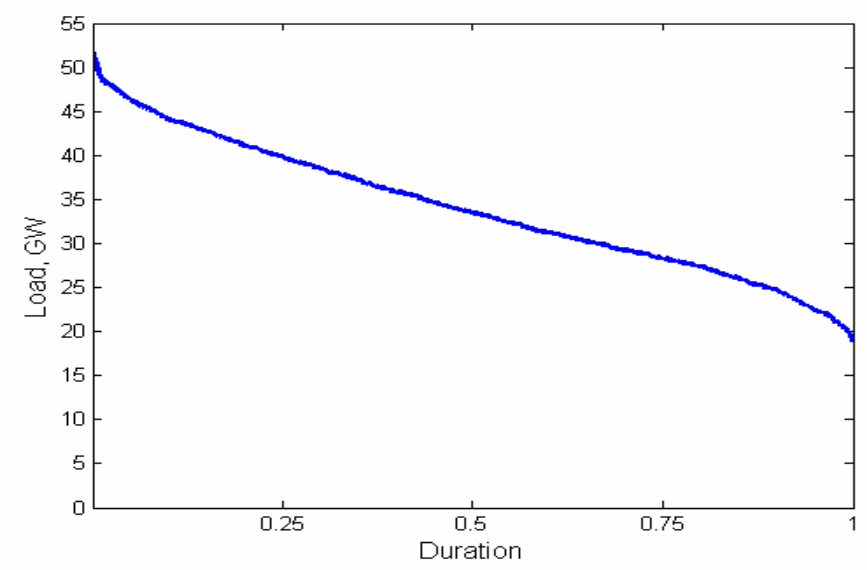

Figure 38: Load duration curve for the PJM 1999 summer market 


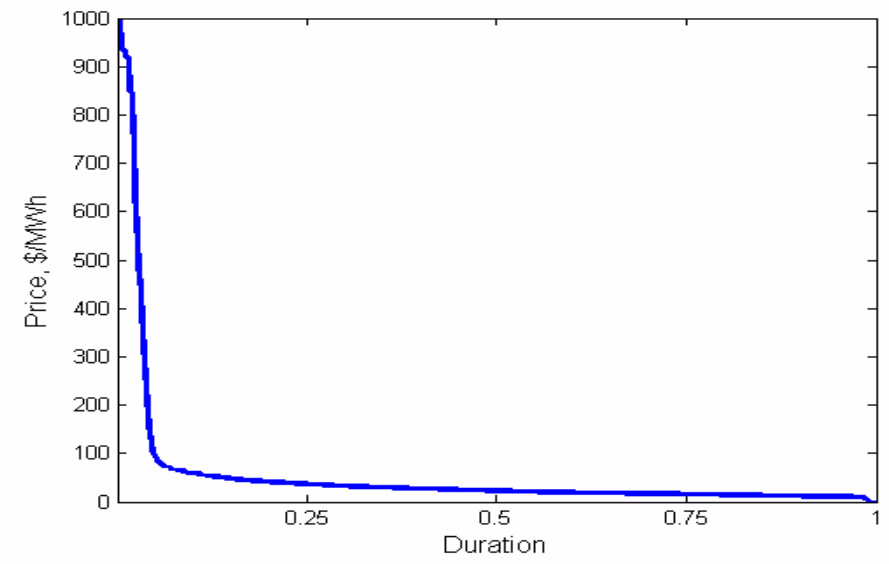

Figure 39: Price duration curve for the PJM 1999 summer market

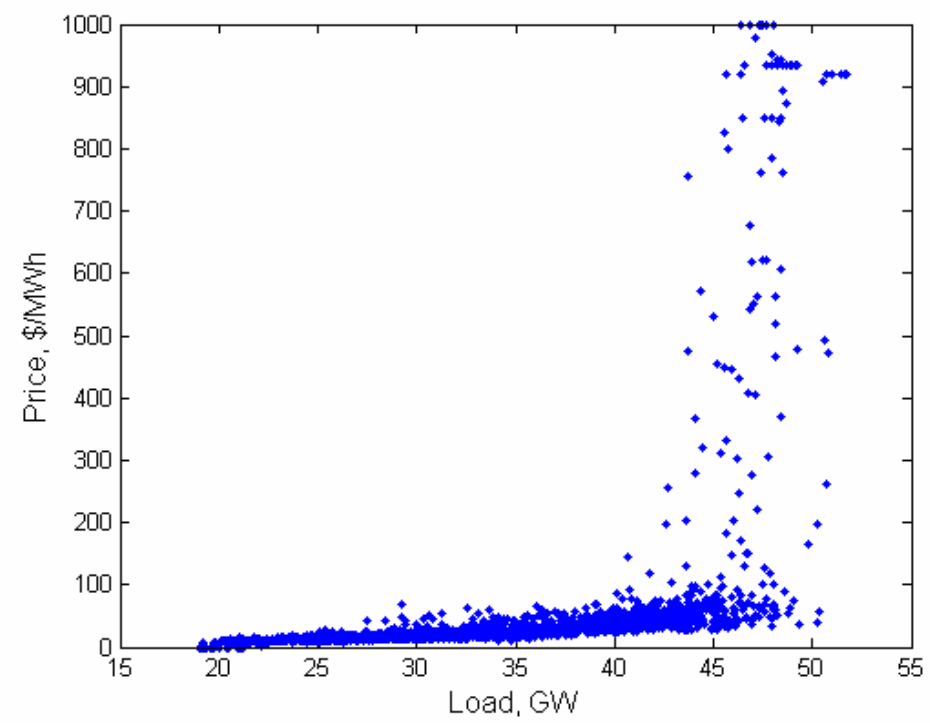

Figure 40: Supply curve for the PJM 1999 summer market

The weather data includes the hourly temperatures noted in Allentown-PA, PhiladelphiaPA Pittsburgh-PA, Albany-NY, New York-NY, Buffalo-NY, Boston-MA, and Washington-VA. 


\section{Hour Classification}

Determination of peak, pre-peak, and off-peak hours eventually defines the hours when the CAHEM system will be in effect of load control. The hour classification is solely determined based upon customer preferences. As previously explained, for the sake of generality of customer preferences, the following three modes of operations have been defined - Super-save Mode, Economy Mode, and Comfort Mode, depending upon the comfort preferences. The hour classification for the PJM 1999 summer data with these three modes is shown in Figure 41.

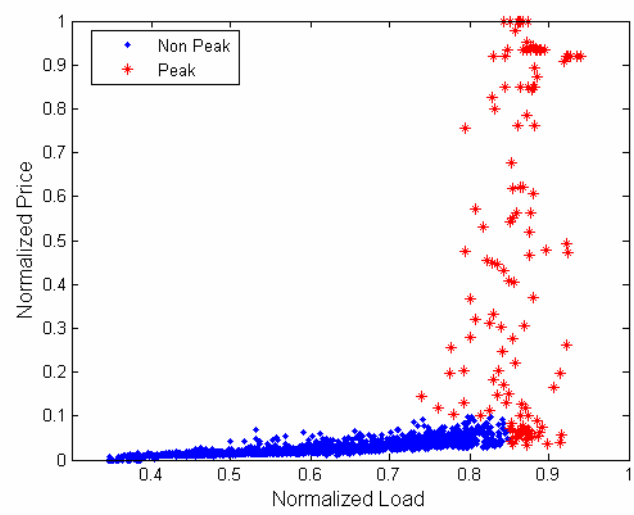

(a) Super-Save Mode

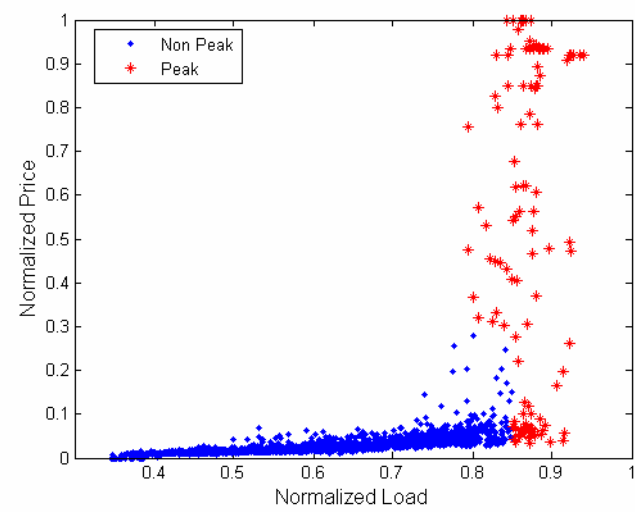

(b) Economy Mode

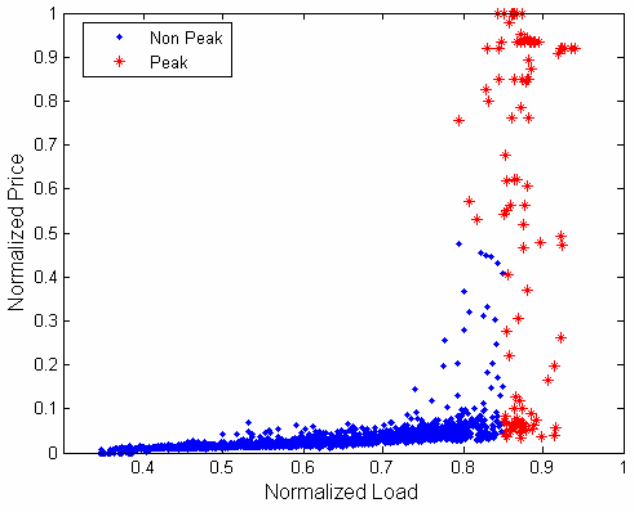

(c) Comfort Mode

Figure 41: Hour Classification 


\subsection{Results at Individual House-level}

The impacts of the CAHEM system on the load profile of an individual housing unit for different modes of operation will be studied. As stated earlier, the extent of the advanced price notification determines the load control strategy depending on the amount of information available for decision making. The hourly load profiles for a prototype house with an hour-ahead notification and an N-hour-ahead notification with $\mathrm{N}$ equal to 12 are presented in Figure 42 and Figure 43 respectively. The results represented here are for the economy mode.

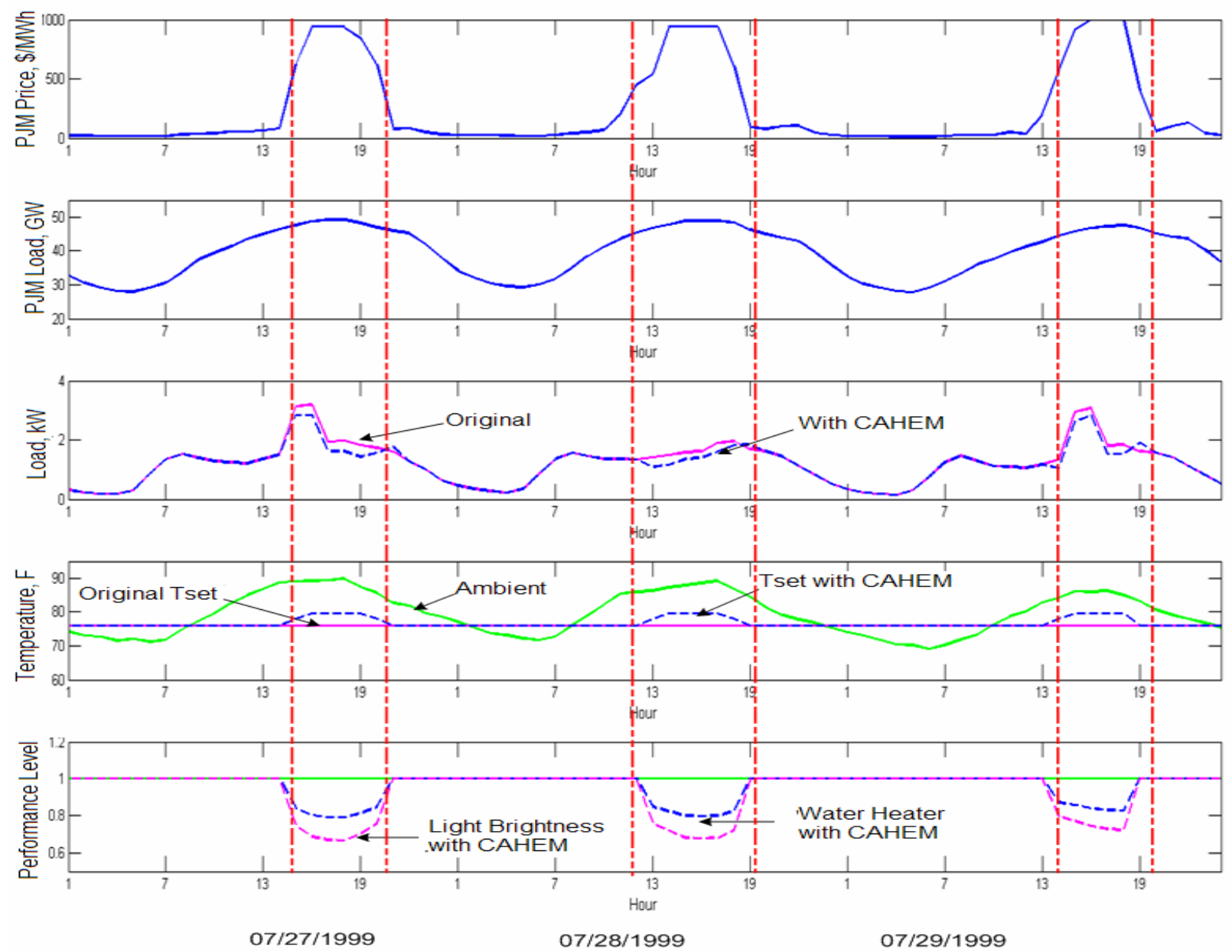

Figure 42: House-level results with Hour-Ahead Notification 


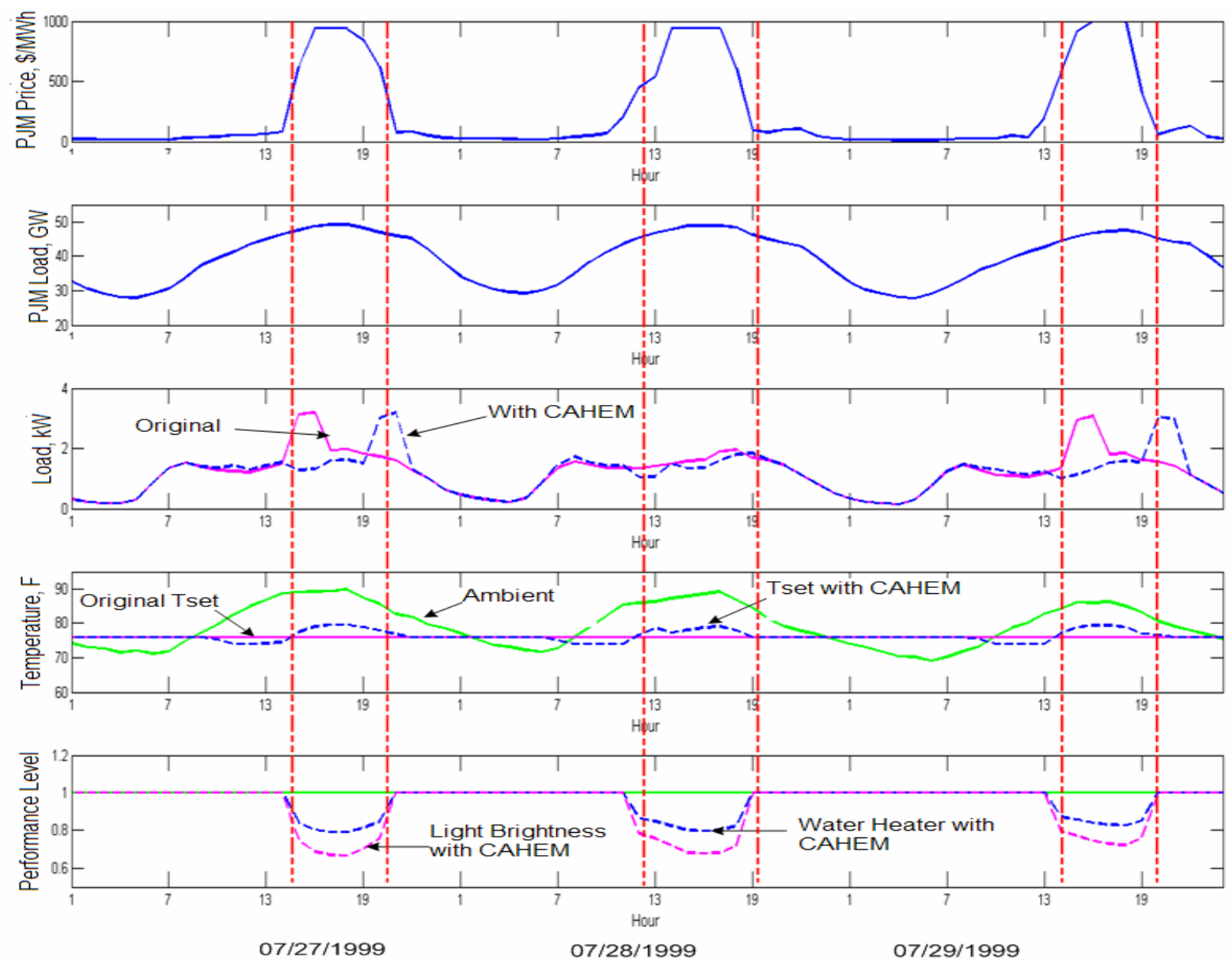

Figure 43: House-level results with N-Hour-Ahead Notification $(\mathrm{N}=12)$

In the case of the hour-ahead notification, the thermostat setpoint is higher than the desired, resulting in a reduced cooling load during the peak hours. A slight increase in electricity consumption at the first off-peak hour that follows the peak hours is due to the extra cooling required to bring the temperature to the desired setpoint. Despite this marginal load increase, the load control with the hour-ahead notification essentially causes load curtailment because of the lack of future price information required for the load shifting.

With N-hour-ahead notification, the thermostat is set to a lower temperature during the pre-peak hour, which results in a higher cooling load. Peak load reduction with an air conditioner is achieved by increasing the thermostat setting during the peak hours. The 
reschedulable services offer a great opportunity to shift the load from peak to off-peak hours.

\subsection{Results at Aggregate-level}

In the aggregate-level results, the simulation results for the PJM territory are presented. The PJM market served approximately 33 million houses in 1999. This number is obtained from the PJM territory map [37] state-wise population provided by the US Census Bureau [38].

It is computationally impossible to individually model each house with the different construction properties and customer preferences. Therefore, 100 different of classes of houses representing the complete residential sector were considered. The housing units in the given territory are equally distributed into 100 different classes. The variety of house construction properties is incorporated by distributing their values with either uniform or Gaussian probability within the expected range. As the floor area and energy consumptions have a high correlation, the distribution of the floor area for the house classes is shown in Figure 44.

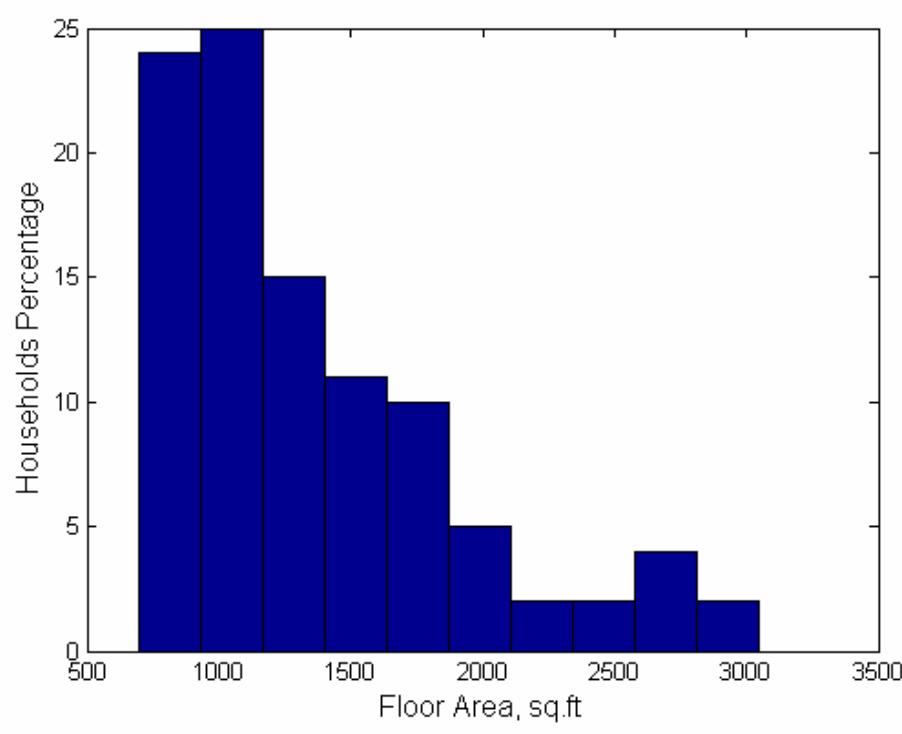

Figure 44: Distribution of floor area of house classes 
Similarly, appliance parameters, such as efficiency, capacity, and operational preferences, vary from house to house within the expected range. These parameters are also modeled with uniform or Gaussian distribution.

The following four scenarios for the distribution of customer preferences among the customer community are considered.

1. 'Super-Save': All households operating in 'Super-Save' mode.

2. 'Economy': All households operating in 'Economy' mode.

3. 'Comfort': All households operating in 'Comfort' mode.

4. 'Random': Operating mode for each household is randomly chosen from the above three modes, assuming operating modes have uniform distribution.

The effects of large-scale implementation of the CAHEM system on the aggregate system load are represented with the help of a load-duration curve as shown in Figure 45 and Figure 46. The load duration curves for the various modes are plotted, assuming that $50 \%$ of the total housing units are equipped with the CAHEM system and are able to respond to the price signals.

A set of load duration curves for the PJM 1999 market with the hour-ahead notification indicates that with $50 \%$ of the houses participating in the CAHEM program, approximately 1 to $3 \%$ peak load reduction (500 to $1500 \mathrm{MW}$ ) is achievable in this scenario. Because of the lack of future information, no load shifting to off-peak hours is possible. As the load shifting with reschedulable services can not be implemented with the hour-ahead notification, the peak load reduction achieved is comparably low. Loadduration curves with the N-hour-ahead notification in Figure 46 show not only better peak load reduction but also load shifting to the hours with relatively low loads. Advance notification results in about 2.5 to $4.25 \%$ load reduction, assuming 50\% CAHEM participation. Table 4 summarizes peak load reduction with the various operating modes. 


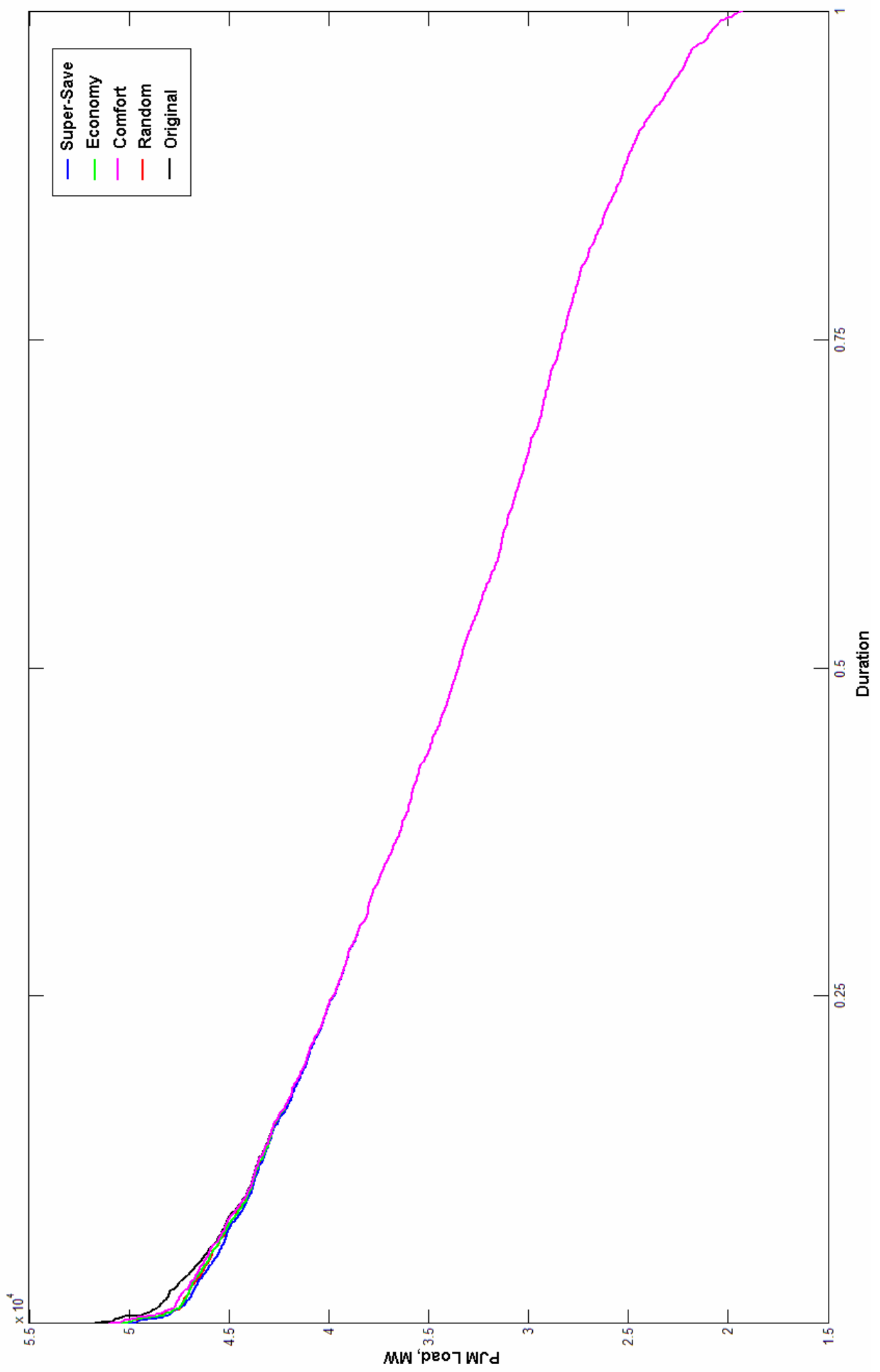

Figure 45: Load duration curves with hour-ahead notification 


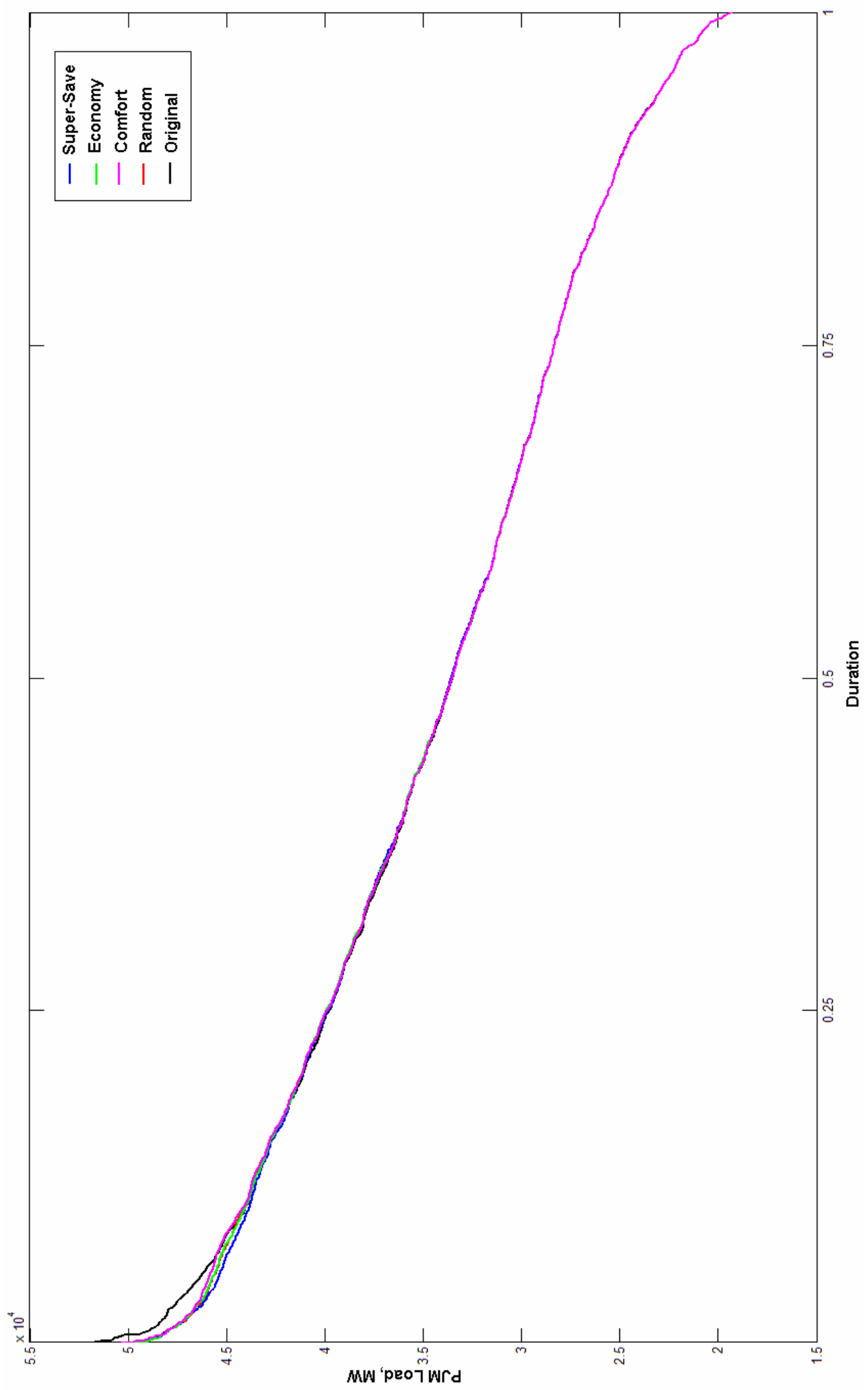

Figure 46: Load duration curves with N-hour-ahead notification ( $\mathrm{N}=12)$ 


\begin{tabular}{|c|c|c|c|c|}
\hline \multirow{2}{*}{$\begin{array}{l}\text { Operating } \\
\text { Mode }\end{array}$} & \multicolumn{2}{|c|}{ Hour-Ahead Notification } & \multicolumn{2}{|c|}{ N-Hour-Ahead Notification } \\
\hline & $\begin{array}{c}\text { Peak Load, } \\
\text { GW }\end{array}$ & $\begin{array}{c}\text { Peak Load } \\
\text { Reduction, \% }\end{array}$ & $\begin{array}{c}\text { Peak Load, } \\
\text { GW }\end{array}$ & $\begin{array}{c}\text { Peak Load } \\
\text { Reduction, \% }\end{array}$ \\
\hline Original & 51.71 & 0 & 51.71 & 0 \\
\hline Random & 50.57 & 2.2 & 49.98 & 3.35 \\
\hline Super-Save & 50.17 & 2.98 & 49.52 & 4.24 \\
\hline Economy & 50.56 & 2.22 & 49.94 & 3.42 \\
\hline Comfort & 51.03 & 1.32 & 50.37 & 2.59 \\
\hline
\end{tabular}

Table 4: Comparison of peak load reduction with different operating modes

The peak load reduction is obtained as an aggregate result of a large number of houses capable of responding to price signals with the CAHEM system. The peak load reduction is dependent upon the total number of houses with the CAHEM system. The CAHEM participation is represented by a fraction of the total households in the territory under consideration.

$$
\text { CAHEM Participation }=\frac{(\# \text { of houses with CAHEM })}{(\text { Total } \# \text { of houses in given territory })}
$$

Figure 47 shows the effect of CAHEM participation on the peak load reduction. With hour-ahead notification, the possible peak load reduction increases with the CAHEM participation. The drop in the load reduction with the Super-Save mode represents an additional cooling load requirement at the end of the peak periods to drop to the desired room temperature.

As the CAHEM participation increases, the load shifting generates the shifted peak in load, which is reflected by the negative slope in Figure 47. The optimal CAHEM participation level for all modes is between 20 to $60 \%$. 


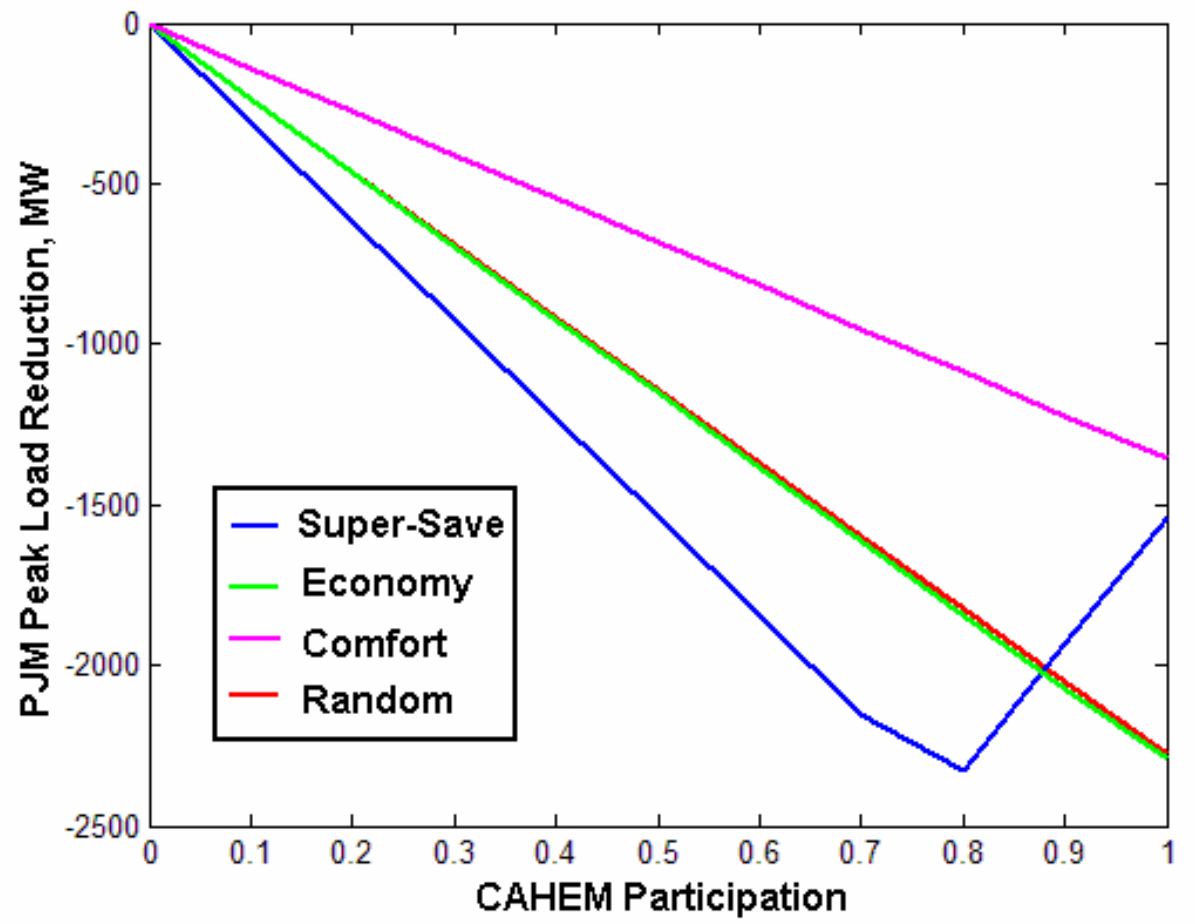

a) Hour-Ahead Notification

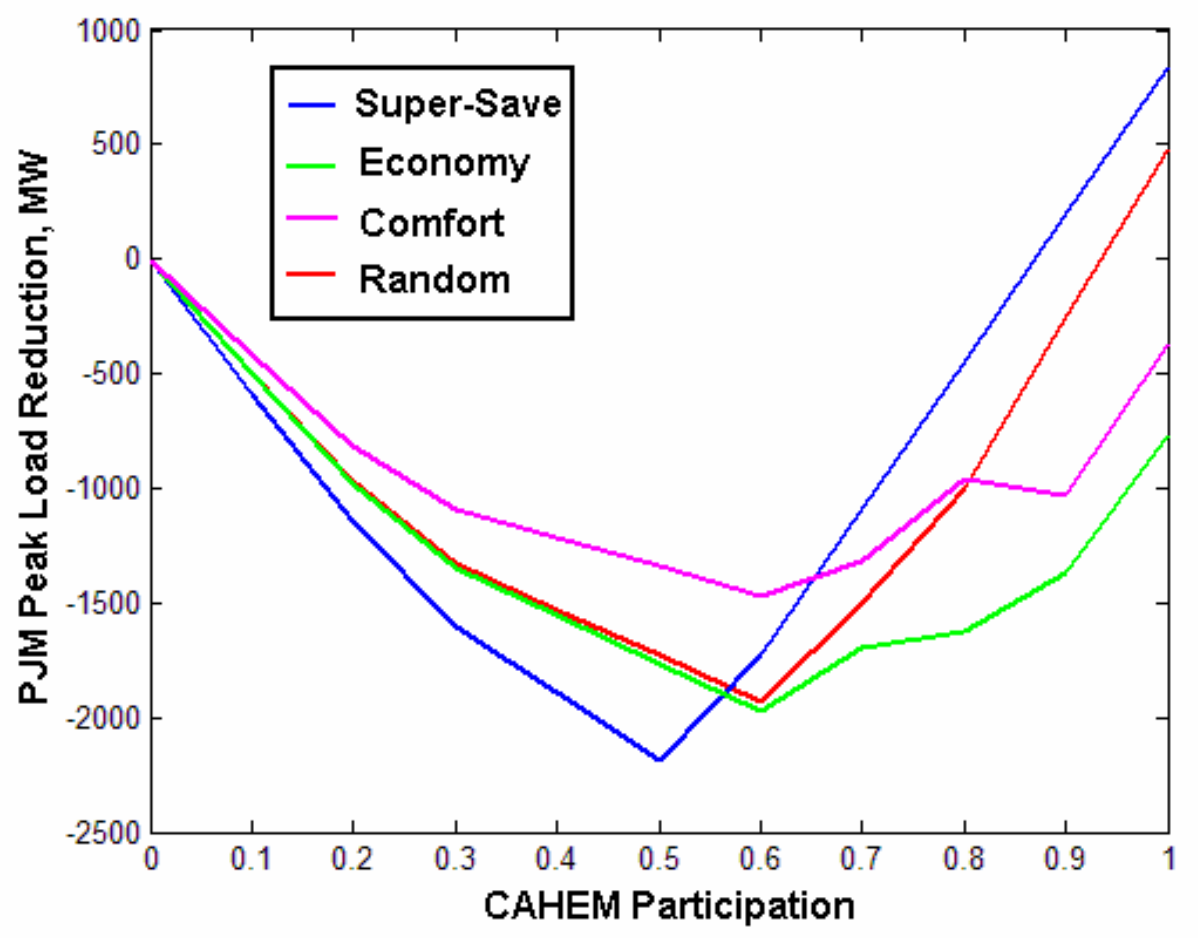

(b) N-Hour-Ahead Notification with $\mathrm{N}=12$

Figure 47: Effect of CAHEM participation on peak load reduction 


\section{Chapter 5}

\section{Conclusions and Future Work}

Peak Load Management has been one of the most critical concerns for the utilities. The load management programs for industrial and commercial customers have been in existence for quite a long time. Extending these programs to residential customers will definitely broaden the scope of the peak load management programs. This thesis investigates the opportunity for managing peak loads with the help of the residential sector.

Conventional direct load control programs are solely under the control of the utility. Many of these programs suffer from high drop-out rates, meaning the overall participation of housing units in the load management program drops over time. Lack of customer involvement in load control decisions is one important reason for the high dropout rates. If the utility wishes to improve the scope of the residential peak load management programs, then the load control needs to be in compliance with customer needs and preferences. The user interface developed in this thesis helps the customer custom-operate peak load management programs depending on his/her needs and priorities. Additionally, customers will gain the power of making impromptu changes in operational settings. The sense of satisfaction achieved with the gain of control of one's own electricity consumption will help in not only improving participation of houses in peak load management programs but also reducing the drop-out rate.

This thesis revisits the concept of price responsive load management and examines its potential for residential energy management. The load control algorithm uses spot prices of electricity and load predictions to decide the new operating points of various residential appliances. The fuzzy load controllers developed in this thesis are based on the PJM 1999 data; however, they can be translated to any market by tuning its membership 
functions according to market data. The availability of detailed data was the primary reason for choosing the PJM market of 1999 [5].

The CAHEM system shows potential for load shifting, which can reduce nearly 2 to $3 \%$ of the peak load at the aggregate level. According to Oh, a three percent reduction in peak load has the ability to eliminate price spikes [5]. The peak load reduction with the CAHEM increases the operating reserve, thus improving reliability of the system.

The prototype CAHEM system, including user interface, demonstrates how price responsive control of residential appliances can be achieved. The appliance controllers supporting the X10 home automation standard were used because of their ready availability and comparatively low cost. The hardware control in the CAHEM system can be incorporated in other home automation standards in the future.

Thus, to summarize, the price responsive load management with the CAHEM system shows great potential for achieving peak load management from the residential sector. This research opens new avenues for future generations of residential energy management programs.

For practical implementation of price responsive load management programs on the utility level would require attention to a number of issues which were not addressed in this thesis. This research can be treated as a starting point for exploring new research avenues in this field. Some of these issues are listed in the following section.

1. The availability of a reliable load and price forecasting mechanism was a primary assumption in developing the load shifting algorithm. The reliability of load and price forecasting eventually determines the success of the load shifting algorithm.

2. Though the load control algorithm is based on wholesale electricity prices, the residential customers cannot be charged the same rates, as the retail customer will not have the capacity to face the risks associated with the high volatility of 
wholesale prices. Economists will need to design an efficient hedging or incentive structure to reduce the risk and to entice retail customers towards the price-responsive load management programs. The study should also include the cost benefit analysis of the program.

3. Large scale implementation of such programs will require the infrastructure to establish reliable communication between utilities and retail customers.

4. The CAHEM user interface and hardware support described in this thesis were developed for the sake of demonstration of the concept. The cost-effective and dedicated hardware control will be essential for a wide execution of the load control programs.

5. Pilot or experimental implementation of such programs will provide researchers the opportunities to study customer psychology and preferences from their feedback. It also presents a platform for educating the customers and testing customer acceptability of the program.

These were the issues which were left untouched related to this work. These issues will have to be addressed at a later date. There may be other issues which may have been overlooked. The readers are encouraged to continue this research and visit the above mentioned issues. 


\section{References}

[1] S. Braithwait and K. Eakin, "The Role of Demand Response In Electric Power Market Design" Prepared for Edison Electric Institute, October 2002. [Online] Available at:

http://www.eei.org/industry issues/retail services and delivery/wise energy use/deman d_response/demandresponserole.pdf

[2] Steven Stoft, Power System Economics, Designing Markets for Electricity, IEEE Press, Wiley-Interscience, 2002

[3] J. Osborn and C. Kawann, "Reliability of the U.S. electric system - Recent trends and current issues," LBNL-47043, February 2002, [Online] Available at: http://eetd.lbl.gov/ea/EMS/reports/47043.pdf

[4]S. Borenstein, M. Jaske, and A. Rosenfeld, "Dynamic Pricing, Advanced Metering and Demand Response in Electricity Markets," October 2002 [Online] Available at: http://www.ucei.berkeley.edu/PDF/csemwp105.pdf

[5] H. Oh, "Simulation Methods for Modeling Offer Behavior and Spot Prices in Restructured Markets for Electricity," Ph.D. Dissertation, Cornell University, 2003.

[6] "Supply and Demand," [Online] Available at: http://en.wikipedia.org/wiki/Supply and demand\#vertical supply curve

[7] J. Koomey and R. Brown, "The role of building technologies in reducing and controlling peak electricity demand," LBNL - 49947, September 2002. [Online] Available at: http://enduse.lbl.gov/info/LBNL-49947.pdf

[8] Association of Energy Services Professionals, "Peak Load Management or Demand Response Programs - A Policy Review," [Online] Available at: http://www.goodcents.com/Info/Peak\%20Load\%20Management.pdf

[9] Rate FDT Family Dwelling- Time Of Use Residential Service, Alabama Power Company, [Online] Available at:

http://www.southerncompany.com/alpower/pricing/pdf/fdt.pdf

[10] J. Roop and E. Fathelrahman, "Modeling Electricity Contract Choice: An Agentbased Approach," Pacific Northwest National Laboratory, [Online] Available at: http://gridwise.pnl.gov/docs/pnnl38282.pdf

[11] New Principles for Demand Response Planning, EPRI, Palo Alto, CA 2001. EPP6035/C3047, [Online], Available at: http://www.goodcents.com/Info/New\%20Principles\%20for\%20Demand\%20Response.pdf 
[12] "Home Control Systems," [Online] Available at:

http://www.invensysnetworks.com/Products/default.asp

[13] "MainGate Home/Multi," [Online], Available at:

http://comverge.com/products/maingate.php

[14] K. Wacks, "Utility load management using home automation," IEEE Transactions on Consumer Electronics, Vol. 37, No. 2, May 1991.

[15] K. Davidson, "CEBus: A new standard in Home Automation - The First In-Dept Technical Description” Circuit Cellar, Vol. 10, pg. 20, 1989.

[16] K. Zuberi, "Power Line Carrier (PLC) Communication Systems - Literature Study Report," Master Thesis, Royal Institute of Technology, Stockholm, Sweden, [Online], Available at: http://web.it.kth.se/ iw01_zkh/deliverables/PLC_030815_D02_V01.pdf

[17] Introduction to the LonWorks System, Echelon Corporation. Version 1.0 [Online] Available at: http://osa.echelon.com/Program/LonWorksIntroPDF.htm

[18] "X10 Transmission Theory," [Online] Available at:

http://www.x10.com/technology1.htm

[19] "Review of Residential Electrical Energy Use Data," NAHB Research Center, Inc. [Online], Available at: http://www.toolbase.org/docs/MainNav/Energy/2974_ResEnyUse.pdf

[20] F. C. Schweppe, B. Daryanian and R. D. Tabors, "Algorithms for a Spot Price Responding Residential Load Controller," IEEE Transactions on Power Systems, Vol. 4, No. 2, May 1989, pp. 507-516.

[21] Man-Loong Chan, G. B. Ackerman, E.N. Marsh, J.Y. Yoon, and N. Stoughton, "Simulation-Based Load Synthesis Methodology for Evaluating Load-Management Programs," IEEE Transaction on Power Apparatus and Systems, Vol. PAS-100, No. 4, April 1981, pp. 1771-1778.

[22] A. Pahwa and C.W. Brice,III, "Modeling and System Identification of Residential Air Conditioning Load," IEEE Transaction on Power Apparatus and Systems, Vol. PAS104, No. 6, June 1985, pp. 1418-1425.

[23] C.W. Gellings and R.W. Taylor, "Electric Load Curve Synthesis- A Computer Simulation of an Electric Utility Load Shape," IEEE Transaction on Power Apparatus and Systems, Vol. PAS-100, No. 1, January 1981, pp. 60-65.

[24] Air Quality Strategies: Building Energy Efficiency, [Online], Available at: http://www.dfwcodes.com/energy/EE_Air_Quality_Recommendations_Final.pdf

[25] J. F. Kreider and A. Rabl, Heating and Cooling of Buildings- Design for Efficiency, McGraw-Hill, Inc., 1994, ISBN: 0-07-834776-9. 
[26] R.T. Guttromson, D. P. Chassin and S. E. Widergren, "Residential Energy Resource Models for Distribution Feeder Simulation,” Proc. 2003 IEEE PES General Meeting, Toronto, ON, Canada, 2003.

[27] Hourly Water Heating Calculations, Pacific Gas and Electric Company, [Online], Available at: http://www.energy.ca.gov/title24/2005standards/archive/documents/200205-30_workshop/2002-05-17_WTR_HEAT_CALCS.PDF

[28] J. D. Lutz, X. Liu, and J.E. McMahon, "Modeling Patterns of Hot Water Use in Households," Lawrence Berkley Lab., CA, Nov 01, 1996, Report No. LBL-37805.

[Online], Available at: http://www.osti.gov/bridge/product.biblio.jsp?osti id=451216

[29] Home Laundry and Dishwashing, Tricity Bendix, [Online], Available at: http://www.tricity-bendix.co.uk/TB_Laundry_0704.pdf

[30] Calculating Energy Use, Orange and Rockland Utilities, Inc, [Online], Available at: http://www.oru.com/energyandsafety/energyefficiency/calculatingenergyuse.html

[31] Building America Lighting Worksheets, [Online], Available at:

http://www.eere.energy.gov/buildings/building_america/docs/lighting_020405.xls

[32] R. Hendron, Building America Research Benchmark Definition, Version 3.1, Updated July 14, 2004, NERL/TP-550-36429 [Online] Available at:

http://www.nrel.gov/docs/fy05osti/36429.pdf

[33] P.W. Fairey, "Passive Cooling and Human Comfort," Florida Solar Energy Center Publication DN-5, January 1994. [Online], Available at:

http://edis.ifas.ufl.edu/pdffiles/EH/EH22100.pdf

[34] Psychometric Chart, [Online], Available at:

http://www.rfcafe.com/references/general/psychometric chart.htm

[35] Technology to Support Dynamic Pricing and Demand Response- Metering, Communication and Data Management, California Energy Commission, [Online], Available at: http://ciee.ucop.edu/dretd/demres.pdf

[36] J. Yen and R. Langari, Fuzzy Logic: Intelligence, control, and information, Prentice Hall, ISBN- 0-13-525817-0, 1998.

[37] PJM Territory Served, [Online], Available at: http://pjm.com/about/territoryserved.html

[38] USA QuickFacts from the US Census Bureau, [Online], Available at: http://pjm.com/about/territory-served.html 